Appendix 1. U-Pb analytical data

CORRECTED RATIOS 2,3

CORRECTED AGES (Ma) Best age $\pm 2 \sigma$

\begin{tabular}{|c|c|c|c|c|c|c|c|c|c|c|c|c|c|c|c|c|c|c|c|c|}
\hline Spot Name & $\mathbf{U}(\mathbf{p p m})^{1}$ & Th $(\mathrm{ppm})^{1}$ & $T h / U$ & $207 \mathrm{~Pb} / 206 \mathrm{~Pb}$ & $\pm 2 \sigma^{3}$ & $207 \mathrm{~Pb} / 235 \mathrm{U}$ & $\pm 2 \sigma^{3}$ & $206 \mathrm{~Pb} / 238 \mathrm{U}$ & $\pm 2 \sigma^{3}$ & $208 \mathrm{~Pb} / 232 \mathrm{Th}$ & $\pm 2 \sigma^{3}$ & Rho & $206 \mathrm{~Pb} / 238 \mathrm{U}$ & $\pm 2 \sigma$ & $207 \mathrm{~Pb} / 235 \mathrm{U}$ & $\pm 2 \sigma$ & $207 \mathrm{~Pb} / 206 \mathrm{~Pb}$ & $\pm 2 \sigma$ & & \\
\hline \multicolumn{21}{|l|}{ Sample: ES 11} \\
\hline ES-11 & 1430 & 1022 & 0.71 & 0.046695 & 0.00037 & 0.03182 & 0.000936 & 0.004942 & 0.00014 & N.D. & N.D. & 0.963036 & 31.78 & 0.93 & 31.8 & 0.935427 & 33.63 & 0.19 & 31.78 & 0.93 \\
\hline 28 & 494 & 300 & 0.61 & 0.04827 & 0.00084 & 0.0331 & 0.000968 & 0.005106 & 0.00012 & N.D. & N.D. & 0.803665 & 32.83 & 0.8 & 33.1 & 0.967951 & 111 & 41 & 32.83 & 0.8 \\
\hline 29 & 263 & 160 & 0.61 & 0.046848 & 0.00024 & 0.03149 & 0.000262 & 0.004874 & 0.000032 & N.D. & N.D. & 0.788392 & 31.34 & 0.21 & 31.48 & 0.262154 & 41.5 & 1.2 & 31.34 & 0.21 \\
\hline _ 30 & 190.1 & 107.37 & 0.56 & 0.046699 & 0.00056 & 0.03203 & 0.000674 & 0.004975 & 0.000086 & N.D. & N.D. & 0.821653 & 31.99 & 0.55 & 32.01 & 0.673446 & 33.85 & 0.29 & 31.99 & 0.55 \\
\hline 31 & 357 & 214 & 0.60 & 0.046723 & 0.00049 & 0.03318 & 0.00079 & 0.00515 & 0.00011 & N.D. & N.D. & 0.897636 & 33.11 & 0.73 & 33.14 & 0.788565 & 35.09 & 0.25 & 33.11 & 0.73 \\
\hline 33 & 390.1 & 345.3 & 0.89 & 0.046692 & 0.00055 & 0.03284 & 0.000654 & 0.005103 & 0.000082 & N.D. & N.D. & 0.806514 & 32.81 & 0.53 & 32.81 & 0.653706 & 33.5 & 0.28 & 32.81 & 0.53 \\
\hline$ـ_{34}$ & 757 & 460 & 0.61 & 0.046702 & 0.00034 & 0.03294 & 0.000687 & 0.005115 & 0.0001 & N.D. & N.D. & 0.937134 & 32.89 & 0.67 & 32.91 & 0.686563 & 34.03 & 0.17 & 32.89 & 0.67 \\
\hline 35 & 277 & 170 & 0.61 & 0.0478 & 0.00059 & 0.03263 & 0.000919 & 0.005135 & 0.00013 & N.D. & N.D. & 0.898858 & 33.02 & 0.85 & 32.6 & 0.918183 & 87 & 29 & 33.02 & 0.85 \\
\hline 36 & 450 & 358 & 0.80 & 0.04674 & 0.00083 & 0.03327 & 0.000658 & 0.005162 & 0.000045 & N.D. & N.D. & 0.440673 & 33.19 & 0.29 & 33.23 & 0.657367 & 35.94 & 0.42 & 33.19 & 0.29 \\
\hline${ }_{-}^{37}$ & 274.2 & 205.6 & 0.75 & 0.046752 & 0.0008 & 0.03335 & 0.000861 & 0.005173 & 0.0001 & N.D. & N.D. & 0.748788 & 33.26 & 0.67 & 33.31 & 0.85995 & 36.59 & 0.41 & 33.26 & 0.67 \\
\hline 38 & 111.2 & 47.83 & 0.43 & 0.046711 & 0.00071 & 0.03351 & 0.001036 & 0.005203 & 0.00014 & N.D. & N.D. & 0.870686 & 33.45 & 0.87 & 33.46 & 1.034043 & 34.5 & 0.36 & 33.45 & 0.87 \\
\hline 39 & 175 & 97.6 & 0.56 & 0.046814 & 0.00063 & 0.03328 & 0.000786 & 0.005156 & 0.0001 & N.D. & N.D. & 0.821592 & 33.15 & 0.64 & 33.24 & 0.784678 & 39.76 & 0.32 & 33.15 & 0.64 \\
\hline _.40 & 393.4 & 309.6 & 0.79 & 0.046863 & 0.0002 & 0.03227 & 0.000287 & 0.004994 & 0.000039 & N.D. & N.D. & 0.877513 & 32.11 & 0.25 & 32.25 & 0.287007 & 42.2 & 1 & 32.11 & 0.25 \\
\hline 41 & 94.82 & 48.71 & 0.51 & 0.04702 & 0.0001 & 0.03276 & 0.000478 & 0.005053 & 0.000073 & N.D. & N.D. & 0.989337 & 32.49 & 0.47 & 32.73 & 0.477942 & 50.4 & 5.2 & 32.49 & 0.47 \\
\hline 42 & 108.35 & 97.2 & 0.90 & 0.046903 & 0.00038 & 0.03223 & 0.0005 & 0.004982 & 0.000066 & N.D. & N.D. & 0.853109 & 32.04 & 0.42 & 32.21 & 0.50018 & 44.3 & 1.9 & 32.04 & 0.42 \\
\hline${ }_{-}^{4} 43$ & 248.8 & 113.93 & 0.46 & 0.046725 & 0.0008 & 0.03276 & 0.001174 & 0.005084 & 0.00016 & N.D. & N.D. & 0.87842 & 32.69 & 1 & 32.73 & 1.172623 & 35.19 & 0.41 & 32.69 & 1 \\
\hline$ـ_{4} 44$ & 388.6 & 249.3 & 0.64 & 0.046768 & 0.00014 & 0.03252 & 0.000234 & 0.00505 & 0.000033 & N.D. & N.D. & 0.909146 & 32.47 & 0.21 & 32.54 & 0.233887 & 37.42 & 0.72 & 32.47 & 0.21 \\
\hline _.46 & 144.8 & 71.1 & 0.49 & 0.047044 & 0.00012 & 0.03206 & 0.000783 & 0.004943 & 0.00012 & N.D. & N.D. & 0.994525 & 31.79 & 0.79 & 32.04 & 0.782109 & 51.48 & 0.63 & 31.79 & 0.79 \\
\hline$ـ_{-47}$ & 183.5 & 104.2 & 0.57 & 0.046733 & 0.0004 & 0.03192 & 0.000943 & 0.004953 & 0.00014 & N.D. & N.D. & 0.957082 & 31.85 & 0.91 & 31.9 & 0.942109 & 35.61 & 0.21 & 31.85 & 0.91 \\
\hline _.48 & 111.4 & 57.2 & 0.51 & 0.046745 & 0.00064 & 0.03232 & 0.001005 & 0.005015 & 0.00014 & N.D. & N.D. & 0.897833 & 32.25 & 0.88 & 32.3 & 1.004301 & 36.22 & 0.33 & 32.25 & 0.88 \\
\hline _.49 & 468.4 & 342.2 & 0.73 & 0.046865 & 0.00012 & 0.03234 & 0.00078 & 0.005005 & 0.00012 & N.D. & N.D. & 0.994346 & 32.18 & 0.78 & 32.32 & 0.779312 & 42.35 & 0.61 & 32.18 & 0.78 \\
\hline ES-11_1 & 175 & 86.8 & 0.50 & 0.04716 & 0.00021 & 0.0326 & 0.001049 & 0.00502 & 0.00016 & N.D. & N.D. & 0.990381 & 32.3 & 1 & 32.6 & 1.049135 & 57 & 11 & 32.3 & 1 \\
\hline
\end{tabular}

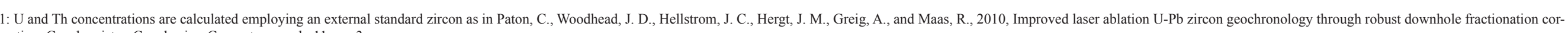
rection. Geochemistry, Geophysics, Geosystems, vol., 11, no. 3.

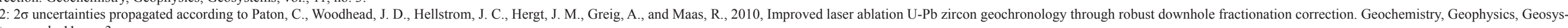

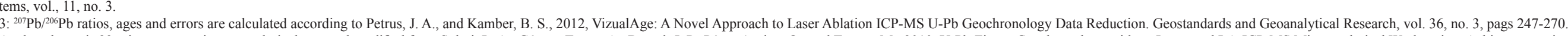

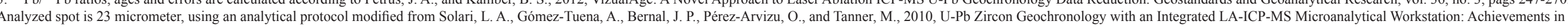
Precision and Accuracy. Geostandards and Geoanalytical Research, vol. 34, no. 1, pags 5-18. Data measured employing a Neptune Plus MC-ICPMS coupled to a Resonetics, Resolution M050 excimer laser workstation. N.D.: Not determined.

CORRECTED RATIOS

CORRECTED AGES (Ma)

Best age $\pm 2 \sigma$

\begin{tabular}{|c|c|c|c|c|c|c|c|c|c|c|c|c|c|c|c|c|c|c|c|c|}
\hline & \multirow[b]{2}{*}{$\mathbf{U}(\mathbf{p p m})^{\S}$} & \multirow[b]{2}{*}{ Th (ppm) $)^{\S}$} & \multirow[b]{2}{*}{$\mathbf{T h} / \mathbf{U}$} & & & \multirow{2}{*}{ (Ma) } & \multirow{2}{*}{ $\pm 2 \sigma$} \\
\hline Spot Name & & & & $207 \mathrm{~Pb} / 206 \mathrm{~Pb}$ & $\pm 2 \sigma^{*}$ & $207 \mathrm{~Pb} / 235 \mathrm{U}$ & $\pm 2 \sigma^{*}$ & $206 \mathrm{~Pb} / 238 \mathrm{U}$ & $\pm 2 \sigma^{*}$ & $208 \mathrm{~Pb} / 232 \mathrm{Th}$ & $\pm 2 \sigma^{*}$ & Rho & $206 \mathrm{~Pb} / 238 \mathrm{U}$ & $\pm 2 \sigma$ & $207 \mathrm{~Pb} / 235 \mathrm{U}$ & $\pm 2 \sigma$ & $207 \mathrm{~Pb} / 206 \mathrm{~Pb}$ & $\pm 2 \sigma$ & & \\
\hline Sample: $\mathrm{MdCh} 0$ & & & & & & & & & & & & & & & & & & & & \\
\hline Z_1_Mdch003 & 268 & 106 & 0.40 & 0.121 & 0.014 & 0.0595 & 0.0076 & 0.00364 & 0.00019 & 0.00266 & 0.00042 & 0.27411 & 23.4 & 1.2 & 58.6 & 7.2 & 1970 & 120 & 23.4 & 1.2 \\
\hline Zircon_2 & 199 & 126 & 0.63 & 0.177 & 0.012 & 0.084 & 0.0067 & 0.00345 & 0.0002 & 0.00213 & 0.00031 & 0.18919 & 22.2 & 1.3 & 81.8 & 6.3 & 2558 & 68 & 22.2 & 1.3 \\
\hline Zircon 4 & 350 & 215 & 0.61 & 0.0937 & 0.0064 & 0.0504 & 0.004 & 0.0039 & 0.00019 & 0.00165 & 0.00021 & 0.613846 & 25.1 & 1.2 & 49.9 & 3.8 & 1507 & 87 & 25.1 & 1.2 \\
\hline
\end{tabular}




\begin{tabular}{|c|c|c|c|c|c|c|c|c|c|c|c|c|c|c|c|c|c|c|c|c|}
\hline Zircon_6 & 289 & 312 & 1.08 & 0.114 & 0.012 & 0.0566 & 0.0071 & 0.00356 & 0.0002 & 0.00151 & 0.00029 & 0.16472 & 22.9 & 1.3 & 58.4 & 7.1 & 1930 & 110 & 22.9 & 1.3 \\
\hline Zircon_7 & 152 & 106 & 0.70 & 0.357 & 0.026 & 0.257 & 0.045 & 0.00537 & 0.00051 & 0.0064 & 0.0012 & 0.37177 & 34.5 & 3.3 & 232 & 32 & 3760 & 80 & 34.5 & 3.3 \\
\hline Zircon_8 & 65 & 59 & 0.91 & 0.227 & 0.036 & 0.139 & 0.025 & 0.00443 & 0.0003 & 0.002 & 0.00044 & 0.376524 & 28.5 & 1.9 & 131 & 21 & 3010 & 180 & 28.5 & 1.9 \\
\hline Zircon_9 & 75 & 25 & 0.33 & 0.152 & 0.031 & 0.097 & 0.025 & 0.0046 & 0.00033 & 0.0032 & 0.001 & 0.09894 & 29.6 & 2.1 & 94 & 22 & 2390 & 210 & 29.6 & 2.1 \\
\hline Zircon_10 & 126 & 61 & 0.48 & 0.093 & 0.018 & 0.06 & 0.013 & 0.00469 & 0.00025 & 0.00215 & 0.0004 & 0.246023 & 30.1 & +.6 & 59 & 13 & 1490 & 200 & 30.1 & 1.6 \\
\hline Zircon_11 & 67 & 21 & 0.31 & 0.057 & 0.02 & 0.027 & 0.014 & 0.00356 & 0.00025 & 0.00234 & 0.00041 & 0.135433 & 22.9 & 1.6 & 27 & 13 & 890 & 240 & 22.9 & 1.6 \\
\hline Zircon_12 & 135 & 51 & 0.38 & 0.063 & 0.015 & 0.0302 & 0.0072 & 0.00348 & 0.00019 & 0.00142 & 0.00024 & 0.229007 & 22.4 & 1.2 & 30.1 & 7 & 1000 & 170 & 22.4 & 1.2 \\
\hline Zircon_16 & 110 & 53 & 0.48 & 0.131 & 0.039 & 0.077 & 0.054 & 0.00461 & 0.00074 & 0.0022 & 0.0011 & 0.2142 & 29.7 & 4.7 & 75 & 41 & 2110 & 240 & 29.7 & 4.7 \\
\hline Zircon_18 & 26 & 22 & 0.85 & 0.195 & 0.062 & 0.155 & 0.051 & 0.00588 & 0.00056 & 0.00252 & 0.00073 & 0.289449 & 37.8 & 3.6 & 143 & 41 & 2910 & 240 & 37.8 & 3.6 \\
\hline Zircon_19 & 74 & 41 & 0.55 & 0.095 & 0.017 & 0.058 & 0.011 & 0.00449 & 0.00029 & 0.0015 & 0.00028 & 0.340555 & 28.9 & 1.9 & 57 & 10 & 1700 & 140 & 28.9 & 1.9 \\
\hline Zircon_21 & 185 & 65 & 0.35 & 0.113 & 0.017 & 0.0583 & 0.0093 & 0.00401 & 0.00022 & 0.00204 & 0.00029 & 0.091803 & 25.8 & 1.4 & 57.2 & 8.9 & 1850 & 120 & 25.8 & 1.4 \\
\hline Zircon_23 & 120 & 37 & 0.31 & 0.144 & 0.041 & 0.086 & 0.044 & 0.00425 & 0.00052 & 0.0028 & 0.0011 & 0.239144 & 27.3 & 3.3 & 83 & 36 & 2350 & 300 & 27.3 & 3.3 \\
\hline Zircon_24 & 150 & 61 & 0.41 & 0.057 & 0.014 & 0.027 & 0.0066 & 0.00359 & 0.00022 & 0.00133 & 0.00019 & 0.250696 & 23.1 & 1.4 & 26.9 & 6.4 & 1190 & 180 & 23.1 & 1.4 \\
\hline Zircon_26 & 109 & 52 & 0.48 & 0.101 & 0.03 & 0.055 & 0.02 & 0.00411 & 0.00027 & 0.00163 & 0.00037 & 0.180657 & 26.5 & 1.7 & 54 & 19 & 1650 & 280 & 26.5 & 1.7 \\
\hline Zircon_27 & 125 & 42 & 0.34 & 0.087 & 0.02 & 0.046 & 0.011 & 0.00411 & 0.00025 & 0.00165 & 0.00036 & 0.009139 & 26.4 & 1.6 & 45 & 10 & 1380 & 200 & 26.4 & 1.6 \\
\hline
\end{tabular}

CORRECTED RATIOS

CORRECTED AGES (Ma)

\begin{tabular}{|c|c|c|c|c|c|c|c|c|c|c|c|c|c|c|c|c|c|c|c|c|}
\hline Spot Name & $\mathbf{U}(\mathbf{p p m})^{\S}$ & Th (ppm) ${ }^{\S}$ & $\mathbf{T h} / \mathbf{U}$ & $207 \mathrm{~Pb} / 206 \mathrm{~Pb}$ & $\pm 2 \sigma^{*}$ & $207 \mathrm{~Pb} / 235 \mathrm{U}$ & $\pm 2 \sigma^{*}$ & $206 \mathrm{~Pb} / 238 \mathrm{U}$ & $\pm 2 \sigma^{*}$ & $208 \mathrm{~Pb} / 232 \mathrm{Th}$ & $\pm 2 \sigma^{*}$ & Rho & $206 \mathrm{~Pb} / 238 \mathrm{U}$ & $\pm 2 \sigma$ & $207 \mathrm{~Pb} / 235 \mathrm{U}$ & $\pm 2 \sigma$ & $207 \mathrm{~Pb} / 206 \mathrm{~Pb}$ & $\pm 2 \sigma$ & (Ma) & $\pm 2 \sigma$ \\
\hline ample: MdCh 10 & & & & & & & & & & & & & & & & & & & & \\
\hline Z_1_Mdch010 & 641 & 465 & $\mathrm{~S}$ & 0.073 & 0.016 & 0.0392 & 0.0088 & 0.00392 & 0.00012 & 0.00145 & 0.0002 & 0.136364 & 25.25 & 0.79 & 39 & 8.6 & 1010 & 160 & 25.25 & 0.79 \\
\hline Zircon_2 & 663 & 1119 & 1.69 & 0.078 & 0.023 & 0.038 & 0.01 & 0.00389 & 0.00013 & 0.00118 & 0.00013 & 0.126992 & 25.05 & 0.82 & 38 & 10 & 1300 & 180 & 25.05 & 0.82 \\
\hline Zircon_3 & 268 & 368 & 1.37 & 0.083 & 0.013 & 0.0448 & 0.0063 & 0.00395 & 0.00014 & 0.00135 & 0.00016 & 0.252039 & 25.43 & 0.9 & 44.5 & 6.2 & 1340 & 200 & 25.43 & 0.9 \\
\hline Zircon_4 & 407 & 422 & 1.04 & 0.0826 & 0.0082 & 0.0422 & 0.0043 & 0.00368 & 0.00013 & 0.00125 & 0.00015 & 0.24717 & 23.7 & 0.8 & 41.9 & 4.2 & 1290 & 110 & 23.7 & 0.8 \\
\hline Zircon_5 & 225 & 128 & 0.57 & 0.07 & 0.017 & 0.0369 & 0.008 & 0.00384 & 0.00018 & 0.00122 & 0.00019 & 0.216211 & 24.7 & 1.2 & 36.6 & 7.9 & 1000 & 270 & 24.7 & 1.2 \\
\hline Zircon_7 & 417 & 146 & 0.35 & 0.069 & 0.011 & 0.0349 & 0.0056 & 0.00384 & 0.00019 & 0.00118 & 0.0002 & 0.41003 & 24.7 & 1.2 & 34.8 & 5.5 & 900 & 170 & 24.7 & 1.2 \\
\hline Zircon_8 & 653 & 559 & 0.86 & 0.0754 & 0.0058 & 0.0383 & 0.003 & 0.003663 & 0.000096 & 0.00127 & 0.00012 & 0.2315 & 23.57 & 0.61 & 38.2 & 3 & 1112 & 66 & 23.57 & 0.61 \\
\hline Zircon_10 & 375 & 296 & 0.79 & 0.074 & 0.016 & 0.0436 & 0.0097 & 0.00418 & 0.00023 & 0.0015 & 0.00019 & 0.032942 & 26.9 & 1.5 & 43.1 & 9.4 & 1170 & 260 & 26.9 & 1.5 \\
\hline Zircon_11 & 684 & 614 & 0.90 & 0.0653 & 0.0053 & 0.0334 & 0.0028 & 0.00371 & 0.00011 & 0.00123 & 0.00012 & 0.353677 & 23.89 & 0.68 & 33.4 & 2.7 & 840 & 120 & 23.89 & 0.68 \\
\hline Zircon_12 & 767 & 710 & 0.93 & 0.068 & 0.0065 & 0.0356 & 0.0035 & 0.003698 & 0.000097 & 0.00121 & 0.00011 & 0.032454 & 23.8 & 0.62 & 35.5 & 3.5 & 950 & 110 & 23.8 & 0.62 \\
\hline Zircon_13 & 619 & 502 & 0.81 & 0.068 & 0.011 & 0.0356 & 0.0058 & 0.00374 & 0.00012 & 0.00126 & 0.00016 & 0.59854 & 24.05 & 0.76 & 35.4 & 5.7 & 890 & 200 & 24.05 & 0.76 \\
\hline Zircon_14 & 1002 & 951 & 0.95 & 0.0565 & 0.006 & 0.0274 & 0.0028 & 0.00365 & 0.000093 & 0.0011 & 0.00011 & 0.16953 & 23.49 & 0.6 & 27.5 & 2.8 & 460 & 110 & 23.49 & 0.6 \\
\hline Zircon_16 & 466 & 340 & 0.73 & 0.071 & 0.013 & 0.0349 & 0.0064 & 0.00377 & 0.00015 & 0.00121 & 0.00013 & 0.19216 & 24.28 & 0.97 & 34.7 & 6.3 & 1040 & 140 & 24.28 & 0.97 \\
\hline Zircon_17 & 767 & 464 & 0.60 & 0.0755 & 0.0058 & 0.0367 & 0.0029 & 0.003554 & 0.000071 & 0.00127 & 0.00011 & 0.30248 & 22.87 & 0.46 & 36.5 & 2.9 & 1070 & 110 & 22.87 & 0.46 \\
\hline Zircon_18 & 563 & 514 & 0.91 & 0.0667 & 0.0084 & 0.0322 & 0.004 & 0.003548 & 0.000086 & 0.00108 & 0.00011 & 0.195124 & 22.83 & 0.55 & 32.2 & 3.9 & 920 & 110 & 22.83 & 0.55 \\
\hline Zircon_19 & 300 & 371 & 1.24 & 0.078 & 0.011 & 0.0419 & 0.0055 & 0.00403 & 0.00014 & 0.00132 & 0.00012 & 0.264651 & 25.94 & 0.91 & 41.6 & 5.3 & 1230 & 140 & 25.94 & 0.91 \\
\hline Zircon_20 & 355 & 233 & 0.66 & 0.079 & 0.01 & 0.0423 & 0.005 & 0.00398 & 0.00013 & 0.00137 & 0.00014 & 0.276332 & 25.61 & 0.81 & 42 & 4.9 & 1220 & 120 & 25.61 & 0.81 \\
\hline Zircon_21 & 792 & 780 & 0.98 & 0.055 & 0.0044 & 0.0293 & 0.0026 & 0.003842 & 0.000087 & 0.00117 & 0.00011 & 0.2891 & 24.72 & 0.56 & 29.3 & 2.6 & 483 & 81 & 24.72 & 0.56 \\
\hline Zircon_22 & 548 & 548 & 1.00 & 0.0672 & 0.0079 & 0.0328 & 0.0038 & 0.00356 & 0.00012 & 0.00107 & 0.00013 & 0.14098 & 22.9 & 0.75 & 32.7 & 3.8 & 940 & 140 & 22.9 & 0.75 \\
\hline Zircon_24 & 558 & 465 & 0.83 & 0.0584 & 0.0065 & 0.0289 & 0.0031 & 0.003678 & 0.000084 & 0.00113 & 0.00011 & 0.212914 & 23.66 & 0.54 & 28.9 & 3.1 & 600 & 140 & 23.66 & 0.54 \\
\hline
\end{tabular}




\begin{tabular}{|c|c|c|c|c|c|c|c|c|c|c|c|c|c|c|c|c|c|c|c|c|}
\hline Zircon_25 & 585 & 467 & 0.80 & 0.0628 & 0.0073 & 0.0321 & 0.0038 & 0.0037 & 0.00012 & 0.00123 & 0.00012 & 0.17482 & 23.79 & 0.78 & 32 & 3.8 & 700 & 71 & 23.79 & 0.78 \\
\hline Zircon_26 & 214 & 130 & 0.61 & 0.073 & 0.012 & 0.0357 & 0.0066 & 0.00378 & 0.00019 & 0.0015 & 0.00021 & 0.18506 & 24.3 & 1.2 & 36.9 & 6.1 & 890 & 150 & 24.3 & 1.2 \\
\hline Zircon_30 & 554 & 256 & 0.46 & 0.0705 & 0.007 & 0.039 & 0.0038 & 0.00394 & 0.00013 & 0.00159 & 0.00018 & 0.338632 & 25.33 & 0.82 & 38.8 & 3.7 & 920 & 180 & 25.33 & 0.82 \\
\hline Zircon_31 & 1571 & 1499 & 0.95 & 0.0508 & 0.0027 & 0.0257 & 0.0015 & 0.003671 & 0.000079 & 0.001064 & 0.000087 & 0.29948 & 23.62 & 0.51 & 25.7 & 1.5 & 307 & 66 & 23.62 & 0.51 \\
\hline Zircon_33 & 186 & 153 & 0.82 & 0.073 & 0.012 & 0.0424 & 0.0071 & 0.00419 & 0.00014 & 0.00126 & 0.00014 & 0.017831 & 26.98 & 0.87 & 42.1 & 6.9 & 1110 & 170 & 26.98 & 0.87 \\
\hline Zircon_35 & 400 & 438 & 1.10 & 0.057 & 0.011 & 0.0267 & 0.0045 & 0.00361 & 0.00012 & 0.00117 & 0.00012 & 0.19723 & 23.25 & 0.8 & 26.7 & 4.4 & 610 & 180 & 23.25 & 0.8 \\
\hline Zircon_36 & 159 & 115 & 0.72 & 0.073 & 0.021 & 0.045 & 0.013 & 0.00445 & 0.00015 & 0.00154 & 0.00028 & 0.15727 & 28.6 & 0.96 & 44 & 12 & 1140 & 360 & 28.6 & 0.96 \\
\hline Zircon_37 & 605 & 447 & 0.74 & 0.0504 & 0.0072 & 0.0257 & 0.0036 & 0.003771 & 0.000098 & 0.00117 & 0.00013 & 0.21211 & 24.26 & 0.63 & 25.8 & 3.6 & 410 & 160 & 24.26 & 0.63 \\
\hline Zircon_38 & 534 & 459 & 0.86 & 0.0536 & 0.0099 & 0.0266 & 0.0048 & 0.00365 & 0.00017 & 0.00137 & 0.00015 & 0.051363 & 23.5 & 1.1 & 26.7 & 4.8 & 580 & 190 & 23.5 & 1.1 \\
\hline Zircon_39 & 631 & 553 & 0.88 & 0.0532 & 0.0057 & 0.0275 & 0.0033 & 0.00368 & 0.00011 & 0.001116 & 0.000097 & 0.249094 & 23.7 & 0.71 & 27.5 & 3.2 & 460 & 100 & 23.7 & 0.71 \\
\hline Zircon_40 & 489 & 353 & 0.72 & 0.0786 & 0.0091 & 0.0406 & 0.0048 & 0.003784 & 0.000091 & 0.00142 & 0.00015 & 0.203411 & 24.35 & 0.59 & 40.3 & 4.7 & 1160 & 190 & 24.35 & 0.59 \\
\hline
\end{tabular}

CORRECTED RATIOS ${ }^{\dagger, *}$

CORRECTED AGES (Ma)

\begin{tabular}{|c|c|c|c|c|c|c|c|c|c|c|c|c|c|c|c|c|c|c|c|c|}
\hline Spot Name & $\mathbf{U}(\mathbf{p p m})^{\S}$ & Th $(\text { ppm) })^{\S}$ & $\mathbf{T h} / \mathbf{U}$ & $207 \mathrm{~Pb} / 206 \mathrm{~Pb}$ & $\pm 2 \sigma^{*}$ & $207 \mathrm{~Pb} / 235 \mathrm{U}$ & $\pm 2 \sigma^{*}$ & $206 \mathrm{~Pb} / 238 \mathrm{U}$ & $\pm 2 \sigma^{*}$ & $208 \mathrm{~Pb} / 232 \mathrm{Th}$ & $\pm 2 \sigma^{*}$ & Rho & $206 \mathrm{~Pb} / 238 \mathrm{U}$ & $\pm 2 \sigma$ & $207 \mathrm{~Pb} / 235 \mathrm{U}$ & $\pm 2 \sigma$ & $207 \mathrm{~Pb} / 206 \mathrm{~Pb}$ & $\pm 2 \sigma$ & (Ma) & $\pm 2 \sigma$ \\
\hline ample: MdCH 05 & & & & & & & & & & & & & & & & & & & & \\
\hline Z_1_Mdch005 & 568 & 1012 & 1.78 & 0.049 & 0.016 & 0.027 & 0.01 & 0.00393 & 0.00016 & 0.00118 & 0.00021 & 0.39326 & 25.3 & 1 & 26.6 & 9.6 & 200 & 280 & 25.3 & 1 \\
\hline Zircon_2 & 394 & 1336 & 3.39 & 0.0594 & 0.0095 & 0.0332 & 0.0058 & 0.00417 & 0.00014 & 0.00141 & 0.0001 & 0.192177 & 26.81 & 0.93 & 33.2 & 5.6 & 760 & 180 & 26.81 & 0.93 \\
\hline Zircon_4 & 353 & 245 & 0.69 & 0.079 & 0.017 & 0.045 & 0.011 & 0.00407 & 0.0002 & 0.00209 & 0.00048 & 0.201027 & 26.2 & 1.3 & 44 & 11 & 1180 & 280 & 26.2 & 1.3 \\
\hline Zircon_5 & 345 & 210 & 0.61 & 0.0603 & 0.0081 & 0.02549 & 0.00095 & 0.00409 & 0.00015 & 0.00156 & 0.00015 & 0.27632 & 26.28 & 0.99 & 25.56 & 0.94 & 760 & 130 & 26.28 & 0.99 \\
\hline Zircon_6 & 322 & 136 & 0.42 & 0.0872 & 0.0086 & 0.0488 & 0.0051 & 0.00407 & 0.00013 & 0.00141 & 0.00021 & 0.204 & 26.21 & 0.85 & 48.2 & 4.9 & 1450 & 120 & 26.21 & 0.85 \\
\hline Zircon_7 & 448 & 302 & 0.67 & 0.055 & 0.011 & 0.0298 & 0.0074 & 0.00394 & 0.00013 & 0.00121 & 0.00027 & 0.19953 & 25.37 & 0.84 & 29.8 & 7.1 & 430 & 230 & 25.37 & 0.84 \\
\hline Zircon_8 & 455 & 348 & 0.76 & 0.05 & 0.0081 & 0.0277 & 0.0047 & 0.00403 & 0.00011 & 0.00122 & 0.00014 & 0.077458 & 25.91 & 0.7 & 27.7 & 4.6 & 460 & 140 & 25.91 & 0.7 \\
\hline Zircon_9 & 243 & 182 & 0.75 & 0.103 & 0.014 & 0.0578 & 0.0077 & 0.00412 & 0.00015 & 0.00154 & 0.00011 & 0.273295 & 26.5 & 0.98 & 56.8 & 7.4 & 1530 & 210 & 26.5 & 0.98 \\
\hline Zircon_10 & 208 & 95 & 0.46 & 0.052 & 0.012 & 0.0305 & 0.0071 & 0.00417 & 0.00015 & 0.0008 & 0.00022 & 0.073628 & 26.86 & 0.98 & 30.4 & 6.9 & 710 & 210 & 26.86 & 0.98 \\
\hline Zircon_11 & 341 & 155 & 0.45 & 0.0466 & 0.0088 & 0.0251 & 0.005 & 0.00407 & 0.00012 & 0.00139 & 0.00013 & 0.14801 & 26.18 & 0.78 & 25.9 & 4.9 & 600 & 170 & 26.18 & 0.78 \\
\hline Zircon_12 & 230 & 94 & 0.41 & 0.0483 & 0.0088 & 0.0294 & 0.0056 & 0.00412 & 0.00013 & 0.00158 & 0.00019 & 0.48082 & 26.52 & 0.81 & 29.2 & 5.5 & 820 & 160 & 26.52 & 0.81 \\
\hline Zircon_13 & 318 & 135 & 0.42 & 0.057 & 0.016 & 0.0316 & 0.0088 & 0.00407 & 0.00017 & 0.00156 & 0.00035 & 0.006113 & 26.2 & 1.1 & 31.5 & 8.4 & 620 & 290 & 26.2 & 1.1 \\
\hline Zircon_14 & 423 & 168 & 0.40 & 0.0484 & 0.0053 & 0.0269 & 0.0031 & 0.00408 & 0.0001 & 0.00123 & 0.000097 & 0.098563 & 26.25 & 0.67 & 26.9 & 3 & 328 & 68 & 26.25 & 0.67 \\
\hline Zircon_15 & 1062 & 529 & 0.50 & 0.055 & 0.0057 & 0.0286 & 0.0035 & 0.00391 & 0.0001 & 0.00145 & 0.00012 & 0.19331 & 25.15 & 0.66 & 28.6 & 3.5 & 520 & 150 & 25.15 & 0.66 \\
\hline Zircon_16 & 327 & 173 & 0.53 & 0.0557 & 0.0081 & 0.0305 & 0.0044 & 0.003993 & 0.000095 & 0.001257 & 0.000099 & 0.164919 & 25.69 & 0.61 & 29.8 & 4.3 & 730 & 110 & 25.69 & 0.61 \\
\hline Zircon_18 & 689 & 274 & 0.40 & 0.0479 & 0.0041 & 0.0261 & 0.0026 & 0.00397 & 0.00012 & 0.001214 & 0.000082 & 0.31977 & 25.53 & 0.78 & 26.1 & 2.6 & 272 & 81 & 25.53 & 0.78 \\
\hline Zircon_20 & 259 & 101 & 0.39 & 0.0562 & 0.0083 & 0.0309 & 0.0047 & 0.00407 & 0.00012 & 0.00118 & 0.00014 & 0.193842 & 26.17 & 0.76 & 30.8 & 4.6 & 760 & 110 & 26.17 & 0.76 \\
\hline Zircon_21 & 388 & 195 & 0.50 & 0.095 & 0.01 & 0.0568 & 0.0062 & 0.00417 & 0.00014 & 0.00224 & 0.00016 & 0.28641 & 26.85 & 0.9 & 56 & 6 & 1630 & 170 & 26.85 & 0.9 \\
\hline Zircon_22 & 511 & 393 & 0.77 & 0.0621 & 0.0081 & 0.0335 & 0.0047 & 0.00405 & 0.00011 & 0.0014 & 0.00012 & 0.32618 & 26.03 & 0.73 & 33.3 & 4.6 & 880 & 150 & 26.03 & 0.73 \\
\hline Zircon_24 & 474 & 167 & 0.35 & 0.0735 & 0.0073 & 0.0408 & 0.0041 & 0.00406 & 0.00012 & 0.00156 & 0.00012 & 0.294125 & 26.11 & 0.78 & 40.5 & 4 & 959 & 98 & 26.11 & 0.78 \\
\hline Zircon_25 & 464 & 326 & 0.70 & 0.0583 & 0.0083 & 0.0322 & 0.0048 & 0.00407 & 0.00013 & 0.00137 & 0.00012 & 0.096228 & 26.16 & 0.83 & 32.1 & 4.7 & 630 & 110 & 26.16 & 0.83 \\
\hline Zircon_28 & 274 & 98 & 0.36 & 0.0524 & 0.0079 & 0.0284 & 0.0045 & 0.0039 & 0.00013 & 0.00145 & 0.00011 & 0.21037 & 25.11 & 0.81 & 28.4 & 4.4 & 680 & 140 & 25.11 & 0.81 \\
\hline Zircon_29 & 338 & 132 & 0.39 & 0.0574 & 0.0069 & 0.0292 & 0.0035 & 0.00386 & 0.00011 & 0.00126 & 0.00012 & 0.23775 & 24.81 & 0.74 & 29.2 & 3.5 & 580 & 140 & 24.81 & 0.74 \\
\hline Zircon_31 & 287 & 188 & 0.66 & 0.12 & 0.01 & 0.0683 & 0.006 & 0.00409 & 0.00014 & 0.00218 & 0.00021 & 0.28881 & 26.34 & 0.9 & 66.9 & 5.7 & 1907 & 94 & 26.34 & 0.9 \\
\hline
\end{tabular}




\begin{tabular}{|c|c|c|c|c|c|c|c|c|c|c|c|c|c|c|c|c|c|c|c|c|}
\hline Zircon_33 & 598 & 318 & 0.53 & 0.0524 & 0.0063 & 0.0286 & 0.0033 & 0.00393 & 0.00011 & 0.0015 & 0.00011 & 0.242578 & 25.31 & 0.7 & 28.7 & 3.3 & 620 & 130 & 25.31 & 0.7 \\
\hline Zircon_34 & 479 & 289 & 0.60 & 0.0748 & 0.0089 & 0.0414 & 0.0054 & 0.00393 & 0.00012 & 0.00169 & 0.00015 & 0.55662 & 25.26 & 0.8 & 41.1 & 5.2 & 1120 & 140 & 25.26 & 0.8 \\
\hline Zircon_35 & 463 & 328 & 0.71 & 0.084 & 0.012 & 0.0458 & 0.0062 & 0.00393 & 0.00013 & 0.00151 & 0.00015 & 0.244357 & 25.27 & 0.85 & 47.1 & 6.1 & 1490 & 260 & 25.27 & 0.85 \\
\hline Zircon_40 & 555 & 408 & 0.74 & 0.0934 & 0.0094 & 0.0533 & 0.0055 & 0.00414 & 0.00013 & 0.00173 & 0.00016 & 0.304304 & 26.63 & 0.85 & 52.6 & 5.4 & 1590 & 140 & 26.63 & 0.85 \\
\hline
\end{tabular}

CORRECTED RATIOS

CORRECTED AGES (Ma)

Best age \pm 2

\begin{tabular}{|c|c|c|c|c|c|c|c|c|c|c|c|c|c|c|c|c|c|c|c|c|}
\hline Spot Name & $\mathbf{U}(\mathbf{p p m})^{\S}$ & Th (ppm) $)^{\S}$ & $\mathbf{T h} / \mathbf{U}$ & $207 \mathrm{~Pb} / 206 \mathrm{~Pb}$ & $\pm 2 \sigma^{*}$ & $207 \mathrm{~Pb} / 235 \mathrm{U}$ & $\pm 2 \sigma^{*}$ & $206 \mathrm{~Pb} / 238 \mathrm{U}$ & $\pm 2 \sigma^{*}$ & $208 \mathrm{~Pb} / 232 \mathrm{Th}$ & $\pm 2 \sigma^{*}$ & Rho & $206 \mathrm{~Pb} / 238 \mathrm{U}$ & $\pm 2 \sigma$ & $207 \mathrm{~Pb} / 235 \mathrm{U}$ & $\pm 2 \sigma$ & $207 \mathrm{~Pb} / 206 \mathrm{~Pb}$ & $\pm 2 \sigma$ & (a) & \\
\hline \multicolumn{21}{|c|}{ Sample: MicBarr 01} \\
\hline Z_05_micbar01 & 1046 & 544 & 0.52 & 0.0501 & 0.0044 & 0.0221 & 0.002 & 0.003288 & 0.000054 & 0.000997 & 0.00006 & 0.34961 & 21.16 & 0.35 & 22.2 & 2 & 325 & 76 & 21.16 & 0.35 \\
\hline Zircon_08 & 432 & 1050 & 2.43 & 0.0498 & 0.0091 & 0.0233 & 0.0041 & 0.003283 & 0.000085 & 0.00091 & 0.00011 & 0.147136 & 21.13 & 0.54 & 23.4 & 4.1 & 640 & 160 & 21.13 & 0.54 \\
\hline Zircon_09 & 563 & 535 & 0.95 & 0.0493 & 0.0073 & 0.0223 & 0.003 & 0.003328 & 0.000083 & 0.001003 & 0.000057 & 0.185387 & 21.42 & 0.53 & 22.3 & 3 & 420 & 140 & 21.42 & 0.53 \\
\hline Zircon_12 & 343 & 324 & 0.94 & 0.0536 & 0.0081 & 0.0231 & 0.0036 & 0.003159 & 0.000086 & 0.000902 & 0.000071 & 0.174686 & 20.33 & 0.55 & 23.1 & 3.6 & 520 & 140 & 20.33 & 0.55 \\
\hline Zircon_14 & 260 & 175 & 0.67 & 0.061 & 0.01 & 0.0263 & 0.0043 & 0.003303 & 0.000094 & 0.00099 & 0.00009 & 0.2015 & 21.26 & 0.6 & 26.3 & 4.2 & 940 & 160 & 21.26 & 0.6 \\
\hline Zircon_15 & 204 & 142 & 0.70 & 0.056 & 0.013 & 0.0257 & 0.0057 & 0.0032 & 0.00012 & 0.00107 & 0.0001 & 0.15998 & 20.61 & 0.8 & 25.6 & 5.6 & 630 & 250 & 20.61 & 0.8 \\
\hline Zircon_16 & 281 & 208 & 0.74 & 0.0709 & 0.0087 & 0.0322 & 0.004 & 0.00333 & 0.0001 & 0.001059 & 0.000087 & 0.241742 & 21.4 & 0.66 & 32.1 & 3.9 & 1070 & 120 & 21.4 & 0.66 \\
\hline Zircon_17 & 178 & 80 & 0.45 & 0.057 & 0.021 & 0.025 & 0.01 & 0.00317 & 0.00016 & 0.00119 & 0.00013 & 0.06871 & 20.4 & 1 & 25.3 & 9.7 & 1030 & 270 & 20.4 & 1 \\
\hline Zircon_18 & 396 & 448 & 1.13 & 0.0575 & 0.0064 & 0.026 & 0.003 & 0.003154 & 0.000088 & 0.000959 & 0.000065 & 0.241809 & 20.3 & 0.57 & 26 & 3 & 700 & 150 & 20.3 & 0.57 \\
\hline Zircon_19 & 390 & 249 & 0.64 & 0.059 & 0.015 & 0.0243 & 0.0075 & 0.0031 & 0.00016 & 0.00092 & 0.00013 & 0.167226 & 20 & 1 & 24.4 & 7.2 & 550 & 260 & 20 & 1 \\
\hline Zircon_20 & 220 & 118 & 0.54 & 0.055 & 0.023 & 0.022 & 0.011 & 0.00318 & 0.00015 & 0.00096 & 0.00013 & 0.09434 & 20.49 & 0.99 & 22 & 11 & 1080 & 290 & 20.49 & 0.99 \\
\hline Zircon_21 & 361 & 192 & 0.53 & 0.0506 & 0.0086 & 0.0226 & 0.0037 & 0.0032 & 0.00011 & 0.001086 & 0.000091 & 0.38415 & 20.61 & 0.7 & 22.7 & 3.7 & 760 & 170 & 20.61 & 0.7 \\
\hline Zircon_23 & 563 & 327 & 0.58 & 0.0496 & 0.0041 & 0.0212 & 0.0018 & 0.003205 & 0.000081 & 0.000953 & 0.000069 & 0.38357 & 20.63 & 0.52 & 21.3 & 1.8 & 370 & 94 & 20.63 & 0.52 \\
\hline Zircon_24 & 334 & 373 & 1.12 & 0.0479 & 0.007 & 0.0218 & 0.003 & 0.003195 & 0.000079 & 0.000945 & 0.000062 & 0.179677 & 20.57 & 0.51 & 21.8 & 3 & 540 & 110 & 20.57 & 0.51 \\
\hline Zircon_25 & 176 & 116 & 0.66 & 0.049 & 0.012 & 0.023 & 0.0049 & 0.00331 & 0.00013 & 0.001099 & 0.000091 & 0.24316 & 21.32 & 0.81 & 23 & 4.8 & 590 & 190 & 21.32 & 0.81 \\
\hline Zircon_26 & 818 & 452 & 0.55 & 0.0533 & 0.0058 & 0.0232 & 0.0025 & 0.003264 & 0.000081 & 0.001 & 0.000066 & 0.230294 & 21.01 & 0.52 & 23.2 & 2.4 & 630 & 110 & 21.01 & 0.52 \\
\hline Zircon_27 & 295 & 254 & 0.86 & 0.076 & 0.013 & 0.0335 & 0.0055 & 0.00313 & 0.00011 & 0.0011 & 0.000088 & 0.54964 & 20.15 & 0.71 & 33.3 & 5.4 & 1310 & 190 & 20.15 & 0.71 \\
\hline Zircon_28 & 1651 & 879 & 0.53 & 0.0459 & 0.0028 & 0.0209 & 0.0011 & 0.003237 & 0.000042 & 0.001015 & 0.000055 & 0.246525 & 20.84 & 0.27 & 21 & 1.1 & 243 & 75 & 20.84 & 0.27 \\
\hline Zircon_30 & 474 & 240 & 0.51 & 0.0526 & 0.0092 & 0.0231 & 0.004 & 0.003173 & 0.000085 & 0.001003 & 0.000089 & 0.16341 & 20.43 & 0.55 & 23.2 & 3.9 & 760 & 180 & 20.43 & 0.55 \\
\hline Zircon_31 & 845 & 588 & 0.70 & 0.0487 & 0.0066 & 0.0223 & 0.0028 & 0.003355 & 0.00007 & 0.00096 & 0.000061 & 0.16617 & 21.59 & 0.45 & 22.4 & 2.7 & 510 & 110 & 21.59 & 0.45 \\
\hline Zircon_32 & 633 & 340 & 0.54 & 0.0602 & 0.0074 & 0.027 & 0.0033 & 0.003254 & 0.000066 & 0.000984 & 0.00007 & 0.16595 & 20.94 & 0.42 & 27 & 3.2 & 770 & 130 & 20.94 & 0.42 \\
\hline Zircon_33 & 1117 & 555 & 0.50 & 0.0496 & 0.0046 & 0.0205 & 0.002 & 0.003101 & 0.000047 & 0.001115 & 0.000063 & 0.12042 & 19.96 & 0.3 & 21.5 & 2 & 243 & 85 & 19.96 & 0.3 \\
\hline Zircon_34 & 493 & 257 & 0.52 & 0.049 & 0.0069 & 0.0222 & 0.003 & 0.003144 & 0.000083 & 0.001015 & 0.000071 & 0.11453 & 20.23 & 0.53 & 22.3 & 3 & 560 & 120 & 20.23 & 0.53 \\
\hline Zircon_35 & 880 & 618 & 0.70 & 0.0466 & 0.0049 & 0.0204 & 0.002 & 0.003191 & 0.000046 & 0.000973 & 0.000062 & 0.076801 & 20.54 & 0.3 & 20.5 & 2 & 240 & 120 & 20.54 & 0.3 \\
\hline Zircon_36 & 648 & 254 & 0.39 & 0.0515 & 0.0056 & 0.0219 & 0.0022 & 0.0032 & 0.000062 & 0.00102 & 0.000077 & 0.192869 & 20.6 & 0.4 & 22 & 2.2 & 360 & 100 & 20.6 & 0.4 \\
\hline Zircon_37 & 584 & 254 & 0.43 & 0.0475 & 0.0049 & 0.0215 & 0.0019 & 0.003265 & 0.00006 & 0.001114 & 0.000071 & 0.207947 & 21.01 & 0.39 & 21.6 & 1.9 & 514 & 99 & 21.01 & 0.39 \\
\hline Zircon_39 & 361 & 341 & 0.94 & 0.085 & 0.012 & 0.0378 & 0.0049 & 0.00322 & 0.000095 & 0.001058 & 0.000068 & 0.007593 & 20.73 & 0.61 & 37.6 & 4.8 & 1340 & 100 & 20.73 & 0.61 \\
\hline
\end{tabular}


CORRECTED RATIOS ${ }^{\dagger, *}$

CORRECTED AGES (Ma)

\begin{tabular}{|c|c|c|c|c|c|c|c|c|c|c|c|c|c|c|c|c|c|c|c|c|}
\hline \multirow{2}{*}{ Spot Name } & \multirow[b]{2}{*}{$\mathbf{U}(\mathbf{p p m})^{\S}$} & \multirow[b]{2}{*}{ Th $(\mathrm{ppm}) \S$} & \multirow[b]{2}{*}{$\mathbf{T h} / \mathbf{U}$} & & & & & & & & & & & & & & & & \multirow[b]{2}{*}{$\begin{array}{l}\text { Best age } \\
\text { (Ma) }\end{array}$} & \multirow[b]{2}{*}{ $\pm 2 \sigma$} \\
\hline & & & & $207 \mathrm{~Pb} / 206 \mathrm{~Pb}$ & $\pm 2 \sigma^{*}$ & $207 \mathrm{~Pb} / 235 \mathrm{U}$ & $\pm 2 \sigma^{*}$ & $206 \mathrm{~Pb} / 238 \mathrm{U}$ & $\pm 2 \sigma^{*}$ & $208 \mathrm{~Pb} / 232 \mathrm{Th}$ & $\pm 2 \sigma^{*}$ & Rho & $206 \mathrm{~Pb} / 238 \mathrm{U}$ & $\pm 2 \sigma$ & $207 \mathrm{~Pb} / 235 \mathrm{U}$ & $\pm 2 \sigma$ & $207 \mathrm{~Pb} / 206 \mathrm{~Pb}$ & $\pm 2 \sigma$ & & \\
\hline \multicolumn{21}{|c|}{ mple: ROCA F6 } \\
\hline Zircon_2 & 158 & 73 & 0.46 & 0.118 & 0.027 & 0.021 & 0.005 & 0.001429 & 0.000084 & 0.000472 & 0.000087 & 0.04 & 9.2 & 0.54 & 21 & 4.9 & 1970 & 150 & 9.2 & 0.54 \\
\hline Zircon_4 & 269 & 147 & 0.55 & 0.093 & 0.019 & 0.0183 & 0.0037 & 0.001479 & 0.000076 & 0.000556 & 0.000086 & 0.32 & 9.53 & 0.49 & 18.4 & 3.6 & 1610 & 160 & 9.53 & 0.49 \\
\hline Zircon_10 & 385 & 231 & 0.60 & 0.097 & 0.011 & 0.017 & 0.0019 & 0.001246 & 0.000057 & 0.000419 & 0.000045 & 0.08 & 8.03 & $\theta .36$ & 17.1 & 1.9 & 1440 & 110 & 8.03 & 0.36 \\
\hline Zircon_11 & 198 & 109 & 0.55 & 0.071 & 0.018 & 0.0137 & 0.004 & 0.001472 & 0.000082 & 0.000372 & 0.000087 & 0.04 & 9.48 & 0.53 & 13.8 & 3.9 & 1250 & 170 & 9.48 & 0.53 \\
\hline Zircon_17 & 486 & 326 & 0.67 & 0.088 & 0.011 & 0.017 & 0.0025 & 0.001412 & 0.000065 & 0.000549 & 0.000076 & 0.31 & 9.09 & 0.42 & 17.1 & 2.5 & 1490 & 120 & 9.09 & 0.42 \\
\hline Zircon_18 & 215 & 105 & 0.49 & 0.082 & 0.022 & 0.0163 & 0.004 & 0.001473 & 0.00007 & 0.000626 & 0.000081 & 0.19 & 9.49 & 0.45 & 16.3 & 4 & 1470 & 190 & 9.49 & 0.45 \\
\hline Zircon_19 & 181 & 108 & 0.60 & 0.064 & 0.021 & 0.014 & 0.0068 & 0.00157 & 0.0001 & 0.00055 & 0.00028 & 0.10 & 10.09 & 0.66 & 14.1 & 6.7 & 1170 & 380 & 10.09 & 0.66 \\
\hline Zircon_20 & 190 & 101 & 0.53 & 0.07 & 0.027 & 0.0153 & 0.0067 & 0.001533 & 0.000094 & 0.00059 & 0.00027 & 0.18 & 9.87 & 0.61 & 15.3 & 6.8 & 1130 & 300 & 9.87 & 0.61 \\
\hline Zircon_23 & 203 & 90 & 0.44 & 0.064 & 0.022 & 0.0147 & 0.0043 & 0.001514 & 0.000067 & 0.00084 & 0.00011 & 0.05 & 9.76 & 0.43 & 14.8 & 4.3 & 1220 & 250 & 9.76 & 0.43 \\
\hline Zircon_24 & 344 & 227 & 0.66 & 0.077 & 0.013 & 0.014 & 0.0024 & 0.001326 & 0.000065 & 0.000422 & 0.000082 & 0.29 & 8.54 & 0.42 & 14.1 & 2.4 & 1260 & 180 & 8.54 & 0.42 \\
\hline Zircon_25 & 453 & 411 & 0.91 & 0.076 & 0.016 & 0.0147 & 0.0032 & 0.001399 & 0.00007 & 0.000661 & 0.000097 & 0.20 & 9.01 & 0.45 & 14.7 & 3.2 & 1520 & 150 & 9.01 & 0.45 \\
\hline
\end{tabular}

CORRECTED RATIOS ${ }^{\dagger, *}$

CORRECTED AGES (Ma)

\begin{tabular}{|c|c|c|c|c|c|c|c|c|c|c|c|c|c|c|c|c|c|c|c|c|}
\hline \multirow[b]{2}{*}{ Spot Name } & \multirow[b]{2}{*}{$\mathbf{U}(\mathbf{p p m}){ }^{\S}$} & \multirow[b]{2}{*}{ Th (ppm) $)^{\S}$} & \multirow[b]{2}{*}{$\mathrm{Th} / \mathrm{U}$} & & & \multirow{2}{*}{$\begin{array}{l}\text { Best age } \\
\text { (Ma) }\end{array}$} & \multirow{2}{*}{ $\pm 2 \sigma$} \\
\hline & & & & $207 \mathrm{~Pb} / 206 \mathrm{~Pb}$ & $\pm 2 \sigma^{*}$ & $207 \mathrm{~Pb} / 235 \mathrm{U}$ & $\pm 2 \sigma^{*}$ & $206 \mathrm{~Pb} / 238 \mathrm{U}$ & $\pm 2 \sigma^{*}$ & $208 \mathrm{~Pb} / 232 \mathrm{Th}$ & $\pm 2 \sigma^{*}$ & Rho & $206 \mathrm{~Pb} / 238 \mathrm{U}$ & $\pm 2 \sigma$ & $207 \mathrm{~Pb} / 235 \mathrm{U}$ & $\pm 2 \sigma$ & $207 \mathrm{~Pb} / 206 \mathrm{~Pb}$ & $\pm 2 \sigma$ & & \\
\hline ample: Ruiz 34 & & & & & & & & & & & & & & & & & & & & \\
\hline Zircon 68 & 1269 & 1442 & 1.14 & 0.0739 & 0.0061 & 0.0287 & 0.0031 & 0.00284 & 0.000079 & 0.00099 & 0.00012 & 0.47417 & 18.25 & 0.51 & 28.8 & 3 & 1020 & 120 & 18.25 & 0.51 \\
\hline Zircon_69 & 145 & 97 & 0.67 & 0.121 & 0.025 & 0.044 & 0.014 & 0.00304 & 0.0002 & 0.00103 & 0.0004 & 0.16022 & 19.6 & 1.3 & 43 & 12 & 1920 & 160 & 19.6 & 1.3 \\
\hline Zircon_71 & 326 & 286 & 0.88 & 0.0455 & 0.0065 & 0.0182 & 0.0024 & 0.00287 & 0.0001 & 0.00077 & 0.00006 & 0.2211 & 18.47 & 0.65 & 18.3 & 2.4 & 500 & 100 & 18.47 & 0.65 \\
\hline Zircon 72 & 197 & 128 & 0.65 & 0.075 & 0.023 & 0.032 & 0.013 & 0.00304 & 0.00016 & 0.00105 & 0.00033 & 0.47081 & 19.6 & 1 & 32 & 12 & 1250 & 250 & 19.6 & 1 \\
\hline Zircon_73 & 302 & 335 & 1.11 & 0.128 & 0.018 & 0.054 & 0.0098 & 0.00308 & 0.00012 & 0.00115 & 0.0002 & 0.11887 & 19.84 & 0.76 & 53.4 & 9.5 & 2120 & 150 & 19.84 & 0.76 \\
\hline Zircon_74 & 284 & 131 & 0.46 & 0.0591 & 0.0099 & 0.0224 & 0.0038 & 0.00277 & 0.00011 & 0.00091 & 0.0001 & 0.36137 & 17.86 & 0.68 & 22.5 & 3.7 & 850 & 140 & 17.86 & 0.68 \\
\hline Zircon_75 & 259 & 263 & 1.02 & 0.075 & 0.011 & 0.0304 & 0.0043 & 0.00302 & 0.00011 & 0.00105 & 0.000091 & 0.25751 & 19.45 & 0.7 & 30.4 & 4.2 & 1070 & 170 & 19.45 & 0.7 \\
\hline Zircon_76 & 560 & 795 & 1.42 & 0.0599 & 0.0064 & 0.0257 & 0.0028 & 0.00311 & 0.000089 & 0.00118 & 0.000081 & 0.42791 & 20.03 & 0.57 & 25.7 & 2.8 & 710 & 130 & 20.03 & 0.57 \\
\hline Zircon_77 & 384 & 172 & 0.45 & 0.114 & 0.011 & 0.0491 & 0.0052 & 0.00297 & 0.00011 & 0.00193 & 0.0002 & 0.37496 & 19.12 & 0.72 & 48.6 & 5 & 1980 & 110 & 19.12 & 0.72 \\
\hline Zircon_78 & 163 & 136 & 0.83 & 0.114 & 0.013 & 0.0467 & 0.0051 & 0.003 & 0.00011 & 0.00119 & 0.00014 & 0.10562 & 19.28 & 0.72 & 46.2 & 5 & 1990 & 110 & 19.28 & 0.72 \\
\hline Zircon_79 & 137 & 89 & 0.65 & 0.089 & 0.017 & 0.0421 & 0.008 & 0.00333 & 0.00013 & 0.00159 & 0.00016 & 0.10823 & 21.44 & 0.83 & 41.7 & 7.7 & 1510 & 170 & 21.44 & 0.83 \\
\hline Zircon_80 & 254 & 225 & 0.89 & 0.076 & 0.023 & 0.031 & 0.012 & 0.0031 & 0.00014 & 0.00101 & 0.00027 & 0.00173 & 19.95 & 0.87 & 31 & 11 & 1070 & 280 & 19.95 & 0.87 \\
\hline Zircon_82 & 1857 & 779 & 0.42 & 0.0475 & 0.0029 & 0.0233 & 0.0014 & 0.00356 & 0.000083 & 0.00111 & 0.000079 & 0.38791 & 22.91 & 0.53 & 23.4 & 1.4 & 201 & 51 & 22.91 & 0.53 \\
\hline Zircon_84 & 818 & 1448 & 1.77 & 0.0468 & 0.005 & 0.0207 & 0.0026 & 0.00326 & 0.00023 & 0.00088 & 0.000074 & 0.5617 & 21 & 1.4 & 20.8 & 2.6 & 400 & 100 & 21 & 1.4 \\
\hline Zircon_86 & 393 & 342 & 0.87 & 0.1037 & 0.0069 & 0.042 & 0.0029 & 0.00288 & 0.000094 & 0.00127 & 0.000094 & 0.2752 & 18.54 & 0.6 & 41.8 & 2.8 & 1704 & 69 & 18.54 & 0.6 \\
\hline Zircon_88 & 426 & 513 & 1.20 & 0.0946 & 0.0095 & 0.0374 & 0.0039 & 0.0029 & 0.00008 & 0.00119 & 0.000088 & 0.04757 & 18.66 & 0.52 & 37.3 & 3.8 & 1525 & 98 & 18.66 & 0.52 \\
\hline Zircon_89 & 165 & 109 & 0.66 & 0.072 & 0.015 & 0.0303 & 0.0071 & 0.00314 & 0.00015 & 0.00102 & 0.00018 & 0.20387 & 20.23 & 0.95 & 30.3 & 6.9 & 1050 & 240 & 20.23 & 0.95 \\
\hline Zircon_90 & 236 & 252 & 1.07 & 0.063 & 0.026 & 0.024 & 0.019 & 0.00285 & 0.00023 & 0.0009 & 0.00041 & 0.00146 & 18.4 & 1.4 & 24 & 17 & 850 & 330 & 18.4 & 1.4 \\
\hline Zircon_91-2 & 497 & 630 & 1.27 & 0.125 & 0.013 & 0.0521 & 0.0082 & 0.003 & 0.00012 & 0.00134 & 0.00016 & 0.4715 & 19.28 & 0.79 & 51.5 & 7.7 & 2010 & 130 & 19.28 & 0.79 \\
\hline Zircon_92 & 554 & 692 & 1.25 & 0.0486 & 0.0079 & 0.0194 & 0.0033 & 0.00288 & 0.000087 & 0.00099 & 0.000093 & 0.17734 & 18.57 & 0.56 & 19.5 & 3.2 & 260 & 190 & 18.57 & 0.56 \\
\hline
\end{tabular}




\begin{tabular}{|c|c|c|c|c|c|c|c|c|c|c|c|c|c|c|c|c|c|c|c|c|}
\hline Zircon_93 & 301 & 204 & 0.68 & 0.061 & 0.01 & 0.0273 & 0.0053 & 0.00303 & 0.00013 & 0.00101 & 0.00018 & 0.38257 & 19.5 & 0.81 & 27.3 & 5.2 & 960 & 180 & 19.5 & 0.81 \\
\hline Zircon_94 & 461 & 362 & 0.79 & 0.0535 & 0.0064 & 0.0234 & 0.0029 & 0.00322 & 0.00011 & 0.00117 & 0.0001 & 0.27565 & 20.72 & 0.7 & 23.5 & 2.9 & 498 & 94 & 20.72 & 0.7 \\
\hline Zircon_97 & 1021 & 1710 & 1.67 & 0.0515 & 0.0094 & 0.0198 & 0.0039 & 0.00283 & 0.000076 & 0.00095 & 0.000073 & 0.32617 & 18.24 & 0.49 & 19.9 & 3.8 & 350 & 210 & 18.24 & 0.49 \\
\hline Zircon_98 & 331 & 332 & 1.00 & 0.106 & 0.011 & 0.0463 & 0.0056 & 0.00296 & 0.00013 & 0.00127 & 0.00016 & 0.60916 & 19.04 & 0.83 & 45.9 & 5.4 & 1790 & 120 & 19.04 & 0.83 \\
\hline Zircon_99 & 393 & 443 & 1.13 & 0.0515 & 0.008 & 0.019 & 0.0029 & 0.00282 & 0.000087 & 0.00089 & 0.000071 & 0.20198 & 18.17 & 0.56 & 19.1 & 2.9 & 530 & 140 & 18.17 & 0.56 \\
\hline _101_Ruiz34b & 1171 & 380 & 0.32 & 0.077 & 0.025 & 0.03 & 0.012 & 0.00289 & 0.00013 & 0.00181 & 0.00071 & 0.49427 & 18.57 & 0.82 & 30 & 12 & 1180 & 280 & 18.57 & 0.82 \\
\hline
\end{tabular}

CORRECTED RATIOS +,

CORRECTED AGES (Ma)

\begin{tabular}{|c|c|c|c|c|c|c|c|c|c|c|c|c|c|c|c|c|c|c|c|c|}
\hline \multirow[b]{2}{*}{ Spot Name } & \multirow[b]{2}{*}{$\mathbf{U}(\mathbf{p p m})^{\S}$} & \multirow[b]{2}{*}{ Th $(\text { ppm })^{\S}$} & \multirow[b]{2}{*}{$\mathbf{T h} / \mathbf{U}$} & & & \multirow{2}{*}{$\begin{array}{l}\text { Best age } \\
\text { (Ma) }\end{array}$} & \multirow{2}{*}{ $\pm 2 \sigma$} \\
\hline & & & & $207 \mathrm{~Pb} / 206 \mathrm{~Pb}$ & $\pm 2 \sigma^{*}$ & $207 \mathrm{~Pb} / 235 \mathrm{U}$ & $\pm 2 \sigma^{*}$ & $206 \mathrm{~Pb} / 238 \mathrm{U}$ & $\pm 2 \sigma^{*}$ & $208 \mathrm{~Pb} / 232 \mathrm{Th}$ & $\pm 2 \sigma^{*}$ & Rho & $206 \mathrm{~Pb} / 238 \mathrm{U}$ & $\pm 2 \sigma$ & $207 \mathrm{~Pb} / 235 \mathrm{U}$ & $\pm 2 \sigma$ & $207 \mathrm{~Pb} / 206 \mathrm{~Pb}$ & $\pm 2 \sigma$ & & \\
\hline Sample: Ruiz 07 & & & & & & & & & & & & & & & & & & & & \\
\hline Z_01_Ruiz07 & 2689 & 1109 & 0.41 & 0.0524 & 0.0024 & 0.0259 & 0.0014 & 0.003636 & 0.000083 & 0.00121 & 0.000074 & 0.49 & 23.4 & 0.53 & 25.9 & 1.4 & 330 & 52 & 23.4 & 0.53 \\
\hline Zircon_02 & 560 & 238 & 0.43 & 0.0485 & 0.0038 & 0.0255 & 0.0021 & 0.00387 & 0.0001 & 0.001189 & 0.000098 & 0.31 & 24.93 & 0.67 & 25.5 & 2.1 & 294 & 64 & 24.93 & 0.67 \\
\hline Zircon_04 & 350 & 159 & 0.45 & 0.0513 & 0.0029 & 0.0603 & 0.004 & 0.00885 & 0.00021 & 0.00274 & 0.0002 & 0.50 & 56.8 & 1.4 & 59.4 & 3.8 & 356 & 56 & 56.8 & 1.4 \\
\hline Zircon_05 & 661 & 288 & 0.44 & 0.0527 & 0.0045 & 0.0266 & 0.0023 & 0.003698 & 0.000097 & 0.001212 & 0.000098 & 0.30 & 23.8 & 0.62 & 26.6 & 2.3 & 490 & 100 & 23.8 & 0.62 \\
\hline Zircon_07 & 965 & 292 & 0.30 & 0.0481 & 0.0037 & 0.0242 & 0.0019 & 0.003641 & 0.000083 & 0.001238 & 0.000091 & 0.15 & 23.43 & 0.53 & 24.3 & 1.8 & 150 & 110 & 23.43 & 0.53 \\
\hline Zircon_09 & 466 & 217 & 0.47 & 0.055 & 0.012 & 0.0276 & 0.0076 & 0.00359 & 0.00014 & 0.00124 & 0.00025 & 0.14 & 23.07 & 0.89 & 27.6 & 7.3 & 540 & 220 & 23.07 & 0.89 \\
\hline Zircon_10 & 502 & 140 & 0.28 & 0.0497 & 0.0039 & 0.0257 & 0.0021 & 0.00373 & 0.0001 & 0.00123 & 0.0001 & 0.33 & 24.01 & 0.64 & 25.7 & 2.1 & 299 & 84 & 24.01 & 0.64 \\
\hline Zircon_11 & 244 & 163 & 0.67 & 0.0617 & 0.0052 & 0.0314 & 0.0027 & 0.003731 & 0.000095 & 0.001282 & 0.000091 & 0.30 & 24 & 0.61 & 31.3 & 2.7 & 738 & 82 & 24 & 0.61 \\
\hline Zircon_12 & 681 & 206 & 0.30 & 0.0482 & 0.0028 & 0.0236 & 0.0015 & 0.003585 & 0.000087 & 0.0012 & 0.000082 & 0.38 & 23.07 & 0.56 & 23.6 & 1.5 & 206 & 52 & 23.07 & 0.56 \\
\hline Zircon_13 & 541 & 143 & 0.26 & 0.0497 & 0.0038 & 0.0254 & 0.0021 & 0.003807 & 0.000095 & 0.00123 & 0.00011 & 0.41 & 24.49 & 0.61 & 25.5 & 2 & 249 & 76 & 24.49 & 0.61 \\
\hline Zircon_16 & 1105 & 498 & 0.45 & 0.0549 & 0.0028 & 0.028 & 0.0014 & 0.003665 & 0.000085 & 0.001279 & 0.000089 & 0.33 & 23.58 & 0.55 & 28 & 1.4 & 413 & 76 & 23.58 & 0.55 \\
\hline Zircon_20 & 759 & 268 & 0.35 & 0.0501 & 0.0022 & 0.0254 & 0.0013 & 0.003684 & 0.000087 & 0.001194 & 0.000082 & 0.46 & 23.7 & 0.56 & 25.4 & 1.3 & 290 & 51 & 23.7 & 0.56 \\
\hline Zircon_22 & 431 & 181 & 0.42 & 0.0654 & 0.003 & 0.0333 & 0.0019 & 0.00367 & 0.00011 & 0.00136 & 0.0001 & 0.08 & 23.64 & 0.68 & 33.3 & 1.9 & 836 & 82 & 23.64 & 0.68 \\
\hline Zircon_24 & 1280 & 439 & 0.34 & 0.0492 & 0.0022 & 0.0253 & 0.0013 & 0.003665 & 0.000083 & 0.001223 & 0.000085 & 0.23 & 23.58 & 0.53 & 25.4 & 1.3 & 197 & 69 & 23.58 & 0.53 \\
\hline Zircon_25 & 657 & 229 & 0.35 & 0.0525 & 0.0029 & 0.0255 & 0.0017 & 0.00365 & 0.00009 & 0.001184 & 0.000088 & 0.05 & 23.49 & 0.58 & 25.6 & 1.6 & 433 & 63 & 23.49 & 0.58 \\
\hline Zircon_26 & 904 & 432 & 0.48 & 0.0542 & 0.0035 & 0.0271 & 0.002 & 0.003658 & 0.000089 & 0.00121 & 0.00011 & 0.33 & 23.54 & 0.57 & 27.2 & 2 & 387 & 94 & 23.54 & 0.57 \\
\hline Z_30_Ruiz07 & 262 & 176 & 0.67 & 0.0662 & 0.0074 & 0.0334 & 0.0044 & 0.00366 & 0.00012 & 0.00118 & 0.00012 & 0.10 & 23.56 & 0.76 & 33.3 & 4.3 & 840 & 140 & 23.56 & 0.76 \\
\hline
\end{tabular}

CORRECTED RATIOS

CORRECTED AGES (Ma)

\begin{tabular}{|c|c|c|c|c|c|c|c|c|c|c|c|c|c|c|c|c|c|c|c|c|}
\hline \multirow[b]{2}{*}{ Spot Name } & \multirow[b]{2}{*}{$\mathbf{U}(\mathbf{p p m})^{\S}$} & \multirow[b]{2}{*}{ Th $(p p m)^{\S}$} & \multirow[b]{2}{*}{$\mathbf{T h} / \mathbf{U}$} & & & \multirow{2}{*}{$\begin{array}{l}\text { Best age } \\
\text { (Ma) }\end{array}$} & \multirow{2}{*}{ $\pm 2 \sigma$} \\
\hline & & & & $207 \mathrm{~Pb} / 206 \mathrm{~Pb}$ & $\pm 2 \sigma^{*}$ & $207 \mathrm{~Pb} / 235 \mathrm{U}$ & $\pm 2 \sigma^{*}$ & $206 \mathrm{~Pb} / 238 \mathrm{U}$ & $\pm 2 \sigma^{*}$ & $208 \mathrm{~Pb} / 232 \mathrm{Th}$ & $\pm 2 \sigma^{*}$ & Rho & $206 \mathrm{~Pb} / 238 \mathrm{U}$ & $\pm 2 \sigma$ & $207 \mathrm{~Pb} / 235 \mathrm{U}$ & $\pm 2 \sigma$ & $207 \mathrm{~Pb} / 206 \mathrm{~Pb}$ & $\pm 2 \sigma$ & & \\
\hline Sample: Sin 07 & & & & & & & & & & & & & & & & & & & & \\
\hline Zircon_03 & 462 & 217 & 0.47 & 0.0559 & 0.0043 & 0.037 & 0.0035 & 0.00476 & 0.00019 & 0.00156 & 0.00029 & 0.22029 & 30.6 & 1.2 & 36.9 & 3.6 & 435 & 95 & 30.6 & 1.2 \\
\hline Zircon_04 & 107 & 80 & 0.75 & 0.0856 & 0.0091 & 0.0584 & 0.0078 & 0.00502 & 0.00026 & 0.0018 & 0.0004 & 0.387782 & 32.3 & 1.6 & 57.6 & 7.4 & 1380 & 110 & 32.3 & 1.6 \\
\hline Zircon_05 & 405 & 208 & 0.51 & 0.0603 & 0.0044 & 0.0392 & 0.0037 & 0.00493 & 0.00021 & 0.00154 & 0.0003 & 0.451291 & 31.7 & 1.3 & 39 & 3.6 & 568 & 94 & 31.7 & 1.3 \\
\hline Zircon_06 & 153 & 97 & 0.63 & 0.084 & 0.0086 & 0.0587 & 0.0073 & 0.0051 & 0.00026 & 0.00185 & 0.00041 & 0.21422 & 32.8 & 1.6 & 57.8 & 6.9 & 1220 & 110 & 32.8 & 1.6 \\
\hline Zircon_08 & 455 & 239 & 0.53 & 0.0571 & 0.0044 & 0.0371 & 0.0039 & 0.00476 & 0.0002 & 0.00143 & 0.00028 & 0.27876 & 30.6 & 1.3 & 37 & 3.8 & 480 & 110 & 30.6 & 1.3 \\
\hline Zircon_09 & 133 & 109 & 0.82 & 0.128 & 0.018 & 0.096 & 0.016 & 0.00547 & 0.0003 & 0.00273 & 0.00063 & 0.43044 & 35.2 & 1.9 & 93 & 14 & 2080 & 140 & 35.2 & 1.9 \\
\hline Zircon_12 & 204 & 155 & 0.76 & 0.0646 & 0.0056 & 0.0427 & 0.0049 & 0.00493 & 0.00023 & 0.00161 & 0.00031 & 0.11588 & 31.7 & 1.5 & 42.4 & 4.8 & 728 & 98 & 31.7 & 1.5 \\
\hline
\end{tabular}




\begin{tabular}{|c|c|c|c|c|c|c|c|c|c|c|c|c|c|c|c|c|c|c|c|}
\hline Zircon_13 & 1333 & 784 & 0.59 & 0.0619 & 0.0035 & 0.0397 & 0.0031 & 0.00463 & 0.00018 & 0.00191 & 0.00034 & 0.43333 & 29.8 & 1.2 & 39.6 & 3 & 718 & 92 & 29.8 \\
\hline Zircon_14 & 63 & 41 & 0.65 & 0.106 & 0.022 & 0.081 & 0.023 & 0.00531 & 0.00038 & 0.00254 & 0.00095 & 0.252027 & 34.1 & 2.4 & 78 & 22 & 1760 & 230 & 34.1 \\
\hline Zircon_17 & 120 & 62 & 0.52 & 0.085 & 0.023 & 0.057 & 0.025 & 0.00506 & 0.00035 & 0.0021 & 0.0011 & 0.24816 & 32.5 & 2.3 & 56 & 22 & 1280 & 290 & 32.5 \\
\hline Zircon_18 & 479 & 274 & 0.57 & 0.1064 & 0.0057 & 0.0724 & 0.0062 & 0.00504 & 0.00021 & 0.00301 & 0.00056 & 0.13913 & 32.4 & 1.3 & 71 & 5.8 & 1724 & 66 & 32.4 \\
\hline Zircon_19 & 206 & 127 & 0.62 & 0.055 & 0.011 & 0.0369 & 0.0086 & 0.00484 & 0.00024 & 0.00152 & 0.00039 & 0.212762 & 31.1 & 1.6 & 36.8 & 8.2 & 570 & 200 & 31.1 \\
\hline Zircon_20 & 175 & 106 & 0.61 & 0.0491 & 0.0083 & 0.0334 & 0.006 & 0.00485 & 0.00023 & 0.00172 & 0.00029 & 0.263986 & 31.2 & 1.5 & 33.4 & 5.8 & 550 & 130 & 31.2 \\
\hline Zircon_21 & 166 & 100 & 0.60 & 0.128 & 0.013 & 0.093 & 0.012 & 0.00542 & 0.00028 & 0.00286 & 0.00086 & 0.6369 & 34.8 & 1.8 & 90 & 11 & 2050 & 100 & 34.8 \\
\hline Zircon_22 & 256 & 125 & 0.49 & 0.0632 & 0.008 & 0.0431 & 0.0069 & 0.00483 & 0.00025 & 0.00187 & 0.00046 & 0.079303 & 31.1 & 1.6 & 42.7 & 6.6 & 810 & 140 & 31.1 \\
\hline Zircon_23 & 316 & 162 & 0.51 & 0.0519 & 0.004 & 0.0355 & 0.0035 & 0.00489 & 0.00019 & 0.00152 & 0.00027 & 0.29872 & 31.4 & 1.2 & 35.3 & 3.4 & 510 & 110 & 31.4 \\
\hline Zircon_24 & 76 & 41 & 0.54 & 0.097 & 0.011 & 0.0688 & 0.0096 & 0.005 & 0.00031 & 0.00198 & 0.00043 & 0.36673 & 32.2 & 2 & 67.3 & 9.1 & 1660 & 100 & 32.2 \\
\hline Zircon_25 & 140 & 96 & 0.69 & 0.0913 & 0.0095 & 0.0635 & 0.0072 & 0.00497 & 0.00025 & 0.00189 & 0.00038 & 0.25545 & 32 & 1.6 & 62.4 & 6.8 & 1574 & 99 & 32 \\
\hline Zircon_26 & 234 & 237 & 1.01 & 0.128 & 0.02 & 0.087 & 0.02 & 0.00512 & 0.00032 & 0.00254 & 0.00067 & 0.58317 & 32.9 & 2.1 & 85 & 18 & 2080 & 180 & 32.9 \\
\hline Zircon_27 & 329 & 165 & 0.50 & 0.089 & 0.01 & 0.0591 & 0.0089 & 0.00486 & 0.00021 & 0.00237 & 0.00058 & 0.286933 & 31.2 & 1.4 & 58.3 & 8.4 & 1280 & 180 & 31.2 \\
\hline Zircon_29 & 219 & 180 & 0.82 & 0.076 & 0.012 & 0.049 & 0.013 & 0.00487 & 0.0003 & 0.00173 & 0.00046 & 0.31228 & 31.3 & 1.9 & 48 & 12 & 1020 & 170 & 31.3 \\
\hline Zircon_31 & 138 & 65 & 0.47 & 0.096 & 0.013 & 0.065 & 0.013 & 0.00508 & 0.00031 & 0.00242 & 0.00065 & 0.305118 & 32.7 & 2 & 64 & 12 & 1570 & 160 & 32.7 \\
\hline
\end{tabular}

CORRECTED RATIOS ${ }^{\dagger, *}$

CORRECTED AGES (Ma)

\begin{tabular}{|c|c|c|c|c|c|c|c|c|c|c|c|c|c|c|c|c|c|c|c|c|}
\hline Spot Name & $\mathbf{U}(\mathbf{p p m})^{\S}$ & Th (ppm) $)^{\S}$ & $\mathbf{T h} / \mathbf{U}$ & $207 \mathrm{~Pb} / 206 \mathrm{~Pb}$ & $\pm 2 \sigma^{*}$ & $207 \mathrm{~Pb} / 235 \mathrm{U}$ & $\pm 2 \sigma^{*}$ & $206 \mathrm{~Pb} / 238 \mathrm{U}$ & $\pm 2 \sigma^{*}$ & $208 \mathrm{~Pb} / 232 \mathrm{Th}$ & $\pm 2 \sigma^{*}$ & Rho & $206 \mathrm{~Pb} / 238 \mathrm{U}$ & $\pm 2 \sigma$ & $207 \mathrm{~Pb} / 235 \mathrm{U}$ & $\pm 2 \sigma$ & $207 \mathrm{~Pb} / 206 \mathrm{~Pb}$ & $\pm 2 \sigma$ & & $\pm 2 \sigma$ \\
\hline Sample: $\operatorname{Sin} 09$ & & & & & & & & & & & & & & & & & & & & \\
\hline Z_01_SIN_09 & 372 & 502 & 1.35 & 0.0752 & 0.0075 & 0.0318 & 0.0046 & 0.00296 & 0.00024 & 0.00102 & 0.00015 & 0.095503 & 19 & 1.5 & 31.7 & 4.5 & 1190 & 110 & 19 & 1.5 \\
\hline Zircon_02 & 710 & 986 & 1.39 & 0.0524 & 0.0082 & 0.0218 & 0.004 & 0.00297 & 0.00022 & 0.0009 & 0.00014 & 0.403704 & 19.1 & 1.4 & 21.9 & 3.9 & 340 & 170 & 19.1 & 1.4 \\
\hline Zircon_03 & 710 & 898 & 1.26 & 0.0543 & 0.0043 & 0.0234 & 0.0024 & 0.00301 & 0.00023 & 0.001 & 0.00015 & 0.31871 & 19.4 & 1.5 & 23.5 & 2.4 & 409 & 99 & 19.4 & 1.5 \\
\hline Zircon_04 & 781 & 1081 & 1.38 & 0.0536 & 0.0049 & 0.0221 & 0.0023 & 0.00282 & 0.00022 & 0.00094 & 0.00013 & 0.749615 & 18.2 & 1.4 & 22.2 & 2.3 & 564 & 77 & 18.2 & 1.4 \\
\hline Zircon_05 & 563 & 837 & 1.49 & 0.0492 & 0.0044 & 0.0209 & 0.0024 & 0.00287 & 0.00022 & 0.00093 & 0.00013 & 0.667538 & 18.5 & 1.4 & 20.9 & 2.3 & 520 & 62 & 18.5 & 1.4 \\
\hline Zircon_07 & 1730 & 2642 & 1.53 & 0.1001 & 0.0045 & 0.0398 & 0.0035 & 0.00283 & 0.00023 & 0.00113 & 0.00016 & 0.31648 & 18.2 & 1.5 & 39.7 & 3.4 & 1598 & 67 & 18.2 & 1.5 \\
\hline Zircon_08 & 392 & 324 & 0.83 & 0.0705 & 0.0057 & 0.0287 & 0.0031 & 0.0029 & 0.00022 & 0.00093 & 0.00014 & 0.702336 & 18.7 & 1.4 & 28.7 & 3 & 1039 & 91 & 18.7 & 1.4 \\
\hline Zircon_10 & 1023 & 1698 & 1.66 & 0.0467 & 0.0038 & 0.0186 & 0.0019 & 0.00286 & 0.00021 & 0.00092 & 0.00013 & 0.30108 & 18.4 & 1.4 & 18.7 & 1.9 & 302 & 80 & 18.4 & 1.4 \\
\hline Zircon_11 & 576 & 1068 & 1.85 & 0.0581 & 0.0048 & 0.0231 & 0.0025 & 0.00285 & 0.00021 & 0.00087 & 0.00012 & 0.31248 & 18.3 & 1.4 & 23.1 & 2.5 & 526 & 93 & 18.3 & 1.4 \\
\hline Zircon_12 & 1075 & 1374 & 1.28 & 0.0573 & 0.0033 & 0.023 & 0.0024 & 0.00288 & 0.00024 & 0.00095 & 0.00013 & 0.44845 & 18.6 & 1.6 & 23 & 2.4 & 500 & 70 & 18.6 & 1.6 \\
\hline Zircon_13 & 1778 & 2999 & 1.69 & 0.0551 & 0.0035 & 0.0194 & 0.0017 & 0.00249 & 0.00019 & 0.0008 & 0.00011 & 0.870777 & 16 & 1.2 & 19.5 & 1.7 & 368 & 84 & 16 & 1.2 \\
\hline Zircon_14 & 669 & 655 & 0.98 & 0.0691 & 0.0073 & 0.0276 & 0.0035 & 0.00291 & 0.00023 & 0.00095 & 0.00016 & 0.62327 & 18.7 & 1.5 & 27.7 & 3.5 & 870 & 130 & 18.7 & 1.5 \\
\hline Zircon_15 & 732 & 1085 & 1.48 & 0.0735 & 0.0045 & 0.0308 & 0.0026 & 0.00303 & 0.00023 & 0.00106 & 0.00014 & 0.10238 & 19.5 & 1.4 & 30.8 & 2.6 & 1038 & 67 & 19.5 & 1.4 \\
\hline Zircon_16 & 597 & 539 & 0.90 & 0.0667 & 0.0056 & 0.0277 & 0.0031 & 0.00303 & 0.00023 & 0.00102 & 0.00015 & 0.678271 & 19.5 & 1.5 & 27.7 & 3 & 926 & 99 & 19.5 & 1.5 \\
\hline Zircon_17 & 878 & 1115 & 1.27 & 0.0595 & 0.0043 & 0.0233 & 0.0025 & 0.00282 & 0.00021 & 0.00089 & 0.00012 & 0.2669 & 18.2 & 1.4 & 23.4 & 2.4 & 639 & 85 & 18.2 & 1.4 \\
\hline Zircon_18 & 489 & 364 & 0.74 & 0.071 & 0.0079 & 0.0306 & 0.004 & 0.00313 & 0.00025 & 0.00116 & 0.00018 & 0.611022 & 20.1 & 1.6 & 30.6 & 3.9 & 990 & 120 & 20.1 & 1.6 \\
\hline Zircon_21 & 528 & 475 & 0.90 & 0.0541 & 0.005 & 0.0218 & 0.0024 & 0.00292 & 0.00022 & 0.00093 & 0.00014 & 0.684361 & 18.8 & 1.4 & 21.9 & 2.4 & 460 & 110 & 18.8 & 1.4 \\
\hline Zircon_24 & 605 & 945 & 1.56 & 0.0518 & 0.0041 & 0.0208 & 0.0023 & 0.00288 & 0.00022 & 0.00091 & 0.00013 & 0.690821 & 18.5 & 1.4 & 20.8 & 2.3 & 380 & 91 & 18.5 & 1.4 \\
\hline Zircon_25 & 687 & 584 & 0.85 & 0.0529 & 0.0043 & 0.0217 & 0.0023 & 0.00295 & 0.00022 & 0.00091 & 0.00013 & 0.30006 & 19 & 1.4 & 21.8 & 2.3 & 480 & 110 & 19 & 1.4 \\
\hline Zircon_26 & 488 & 411 & 0.84 & 0.0626 & 0.0069 & 0.0257 & 0.0035 & 0.00297 & 0.00024 & 0.00092 & 0.00015 & 0.22272 & 19.1 & 1.5 & 25.8 & 3.4 & 730 & 120 & 19.1 & 1.5 \\
\hline
\end{tabular}




\begin{tabular}{|c|c|c|c|c|c|c|c|c|c|c|c|c|c|c|c|c|c|c|c|c|}
\hline Zircon_27 & 626 & 918 & 1.47 & 0.0709 & 0.0055 & 0.0256 & 0.0025 & 0.00272 & 0.00021 & 0.00087 & 0.00012 & 0.790588 & 17.5 & 1.3 & 25.7 & 2.5 & 898 & 80 & 17.5 & 1.3 \\
\hline Zircon_29 & 1457 & 2143 & 1.47 & 0.0578 & 0.0036 & 0.0211 & 0.0018 & 0.0026 & 0.0002 & 0.00079 & 0.00012 & 0.19951 & 16.8 & 1.3 & 21.2 & 1.8 & 467 & 83 & 16.8 & 1.3 \\
\hline Zircon_31 & 338 & 290 & 0.86 & 0.0704 & 0.0081 & 0.0279 & 0.0044 & 0.00291 & 0.00023 & 0.00096 & 0.00016 & 0.034163 & 18.7 & 1.5 & 27.9 & 4.3 & 1000 & 130 & 18.7 & 1.5 \\
\hline Zircon_32 & 361 & 342 & 0.95 & 0.0668 & 0.0059 & 0.0262 & 0.0031 & 0.00274 & 0.00022 & 0.00088 & 0.00014 & 0.44202 & 17.6 & 1.4 & 26.3 & 3.1 & 910 & 100 & 17.6 & 1.4 \\
\hline Zircon_33 & 589 & 503 & 0.85 & 0.0727 & 0.0055 & 0.0285 & 0.003 & 0.00287 & 0.00021 & 0.00105 & 0.00016 & 0.695122 & 18.5 & 1.4 & 28.5 & 3 & 1047 & 75 & 18.5 & 1.4 \\
\hline Zircon_34 & 870 & 1371 & 1.58 & 0.0528 & 0.0047 & 0.0195 & 0.0023 & 0.0028 & 0.00021 & 0.0009 & 0.00012 & 0.63587 & 18 & 1.3 & 19.6 & 2.3 & 310 & 120 & 18 & 1.3 \\
\hline
\end{tabular}

CORRECTED RATIOS

CORRECTED AGES (Ma)

\begin{tabular}{|c|c|c|c|c|c|c|c|c|c|c|c|c|c|c|c|c|c|c|c|c|}
\hline \multirow[b]{2}{*}{ Spot Name } & \multirow[b]{2}{*}{$\mathbf{U}(\mathbf{p p m})^{\S}$} & \multirow[b]{2}{*}{ Th (ppm) $)^{\S}$} & \multirow[b]{2}{*}{$\mathbf{T h} / \mathbf{U}$} & & & & & & & & & & & & & & & & \multirow{2}{*}{$\begin{array}{l}\text { Best age } \\
\text { (Ma) }\end{array}$} & \multirow{2}{*}{ $\pm 2 \sigma$} \\
\hline & & & & $207 \mathrm{~Pb} / 206 \mathrm{~Pb}$ & $\pm 2 \sigma^{*}$ & $207 \mathrm{~Pb} / 235 \mathrm{U}$ & $\pm 2 \sigma^{*}$ & $206 \mathrm{~Pb} / 238 \mathrm{U}$ & $\pm 2 \sigma^{*}$ & $208 \mathrm{~Pb} / 232 \mathrm{Th}$ & $\pm 2 \sigma^{*}$ & Rho & $206 \mathrm{~Pb} / 238 \mathrm{U}$ & $\pm 2 \sigma$ & $207 \mathrm{~Pb} / 235 \mathrm{U}$ & $\pm 2 \sigma$ & $207 \mathrm{~Pb} / 206 \mathrm{~Pb}$ & $\pm 2 \sigma$ & & \\
\hline \multicolumn{21}{|l|}{ Sample: Sin 18} \\
\hline 01_SIN_18_009 & 305 & 163 & 0.48 & 0.06041 & 0.015349 & 0.03756 & 0.010103 & 0.00451 & 0.000184 & 0.00139 & 0.00007 & 0.15 & 29 & 1.2 & 37 & 10.0 & 618 & 157.0 & 28.1 & 1.2 \\
\hline Zircon_02_010 & 155 & 84 & 0.49 & 0.07424 & 0.010315 & 0.04648 & 0.007079 & 0.00454 & 0.000184 & 0.00137 & 0.00005 & 0.27 & 29.2 & 1.2 & 46 & 7.0 & 1048 & 145.6 & 27.7 & 1.2 \\
\hline Zircon_03_011 & 348 & 158 & 0.41 & 0.05506 & 0.004786 & 0.03349 & 0.003297 & 0.00441 & 0.000133 & 0.00138 & 0.00005 & 0.31 & 28.4 & 0.9 & 33 & 3.2 & 415 & 36.1 & 27.8 & 0.9 \\
\hline Zircon_04_012 & 464 & 207 & 0.40 & 0.05539 & 0.004788 & 0.03319 & 0.003123 & 0.00435 & 0.000118 & 0.00135 & 0.00003 & 0.29 & 28 & 0.8 & 33 & 3.1 & 428 & 37.0 & 27.4 & 0.8 \\
\hline Zircon_05_013 & 149 & 64 & 0.39 & 0.08205 & 0.016716 & 0.0524 & 0.011389 & 0.00463 & 0.000185 & 0.00138 & 0.00007 & 0.18 & 29.8 & 1.2 & 52 & 11.3 & 1247 & 254.1 & 27.9 & 1.2 \\
\hline Zircon_06_015 & 138 & 91 & 0.59 & 0.06191 & 0.010288 & 0.03828 & 0.006817 & 0.00448 & 0.000166 & 0.00138 & 0.00005 & 0.21 & 28.8 & 1.1 & 38 & 6.8 & 671 & 111.5 & 27.9 & 1.1 \\
\hline Zircon_07_016 & 181 & 137 & 0.68 & 0.06449 & 0.009546 & 0.04191 & 0.007033 & 0.00471 & 0.000203 & 0.00144 & 0.00005 & 0.26 & 30.3 & 1.3 & 42 & 7.0 & 758 & 112.2 & 29.2 & 1.3 \\
\hline Zircon_08_017 & 165 & 94 & 0.51 & 0.05676 & 0.008964 & 0.03556 & 0.006055 & 0.00454 & 0.00015 & 0.00141 & 0.00005 & 0.19 & 29.2 & 1.0 & 35 & 6.0 & 482 & 76.1 & 28.5 & 1.0 \\
\hline Zircon_09_018 & 148 & 94 & 0.57 & 0.07648 & 0.01698 & 0.04959 & 0.011834 & 0.0047 & 0.000186 & 0.00141 & 0.00007 & 0.17 & 30.2 & 1.2 & 49 & 11.7 & 1108 & 246.0 & 28.5 & 1.2 \\
\hline Zircon_10_019 & 171 & 126 & 0.66 & 0.06924 & 0.006457 & 0.04393 & 0.004345 & 0.00459 & 0.000151 & 0.00147 & 0.00009 & 0.33 & 29.5 & 1.0 & 44 & 4.4 & 906 & 84.5 & 29.7 & 1.0 \\
\hline Zircon_11_021 & 434 & 316 & 0.65 & 0.0505 & 0.005145 & 0.0321 & 0.003572 & 0.00461 & 0.000122 & 0.00145 & 0.00004 & 0.24 & 29.7 & 0.8 & 32 & 3.6 & 218 & 22.2 & 29.4 & 0.8 \\
\hline Zircon_12_022 & 264 & 175 & 0.59 & 0.0587 & 0.007062 & 0.03735 & 0.00489 & 0.00462 & 0.000136 & 0.00143 & 0.00005 & 0.23 & 29.7 & 0.9 & 37 & 4.8 & 556 & 66.9 & 28.9 & 0.9 \\
\hline Zircon_13_023 & 204 & 86 & 0.38 & 0.06196 & 0.021272 & 0.04043 & 0.01463 & 0.00473 & 0.000203 & 0.00146 & 0.00014 & 0.12 & 30.4 & 1.3 & 40 & 14.5 & 673 & 231.1 & 29 & 1.3 \\
\hline Zircon_14_024 & 283 & 228 & 0.72 & 0.05864 & 0.009465 & 0.03723 & 0.006694 & 0.00461 & 0.000185 & 0.00143 & 0.00005 & 0.22 & 29.6 & 1.2 & 37 & 6.7 & 554 & 89.4 & 28.8 & 1.2 \\
\hline Zircon_16_027 & 178 & 117 & 0.59 & 0.05886 & 0.007553 & 0.03811 & 0.005051 & 0.00469 & 0.000169 & 0.0015 & 0.00009 & 0.27 & 30.2 & 1.1 & 38 & 5.0 & 562 & 72.1 & 30.3 & 1.1 \\
\hline
\end{tabular}

CORRECTED RATIOS ${ }^{*, *}$

CORRECTED AGES (Ma)

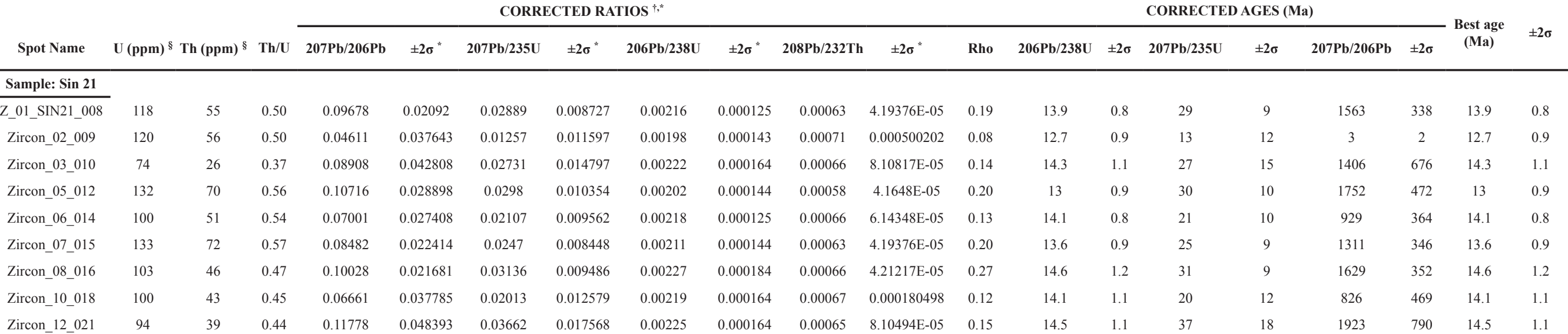




\begin{tabular}{|c|c|c|c|c|c|c|c|c|c|c|c|c|c|c|c|c|c|c|c|c|}
\hline Zircon_14_023 & 127 & 66 & 0.56 & 0.11433 & 0.030019 & 0.03195 & 0.010775 & 0.00203 & 0.000144 & 0.00058 & $4.1648 \mathrm{E}-05$ & 0.21 & 13.1 & 0.9 & 32 & 11 & 1869 & 491 & 13.1 & 0.9 \\
\hline Zircon_15_024 & 128 & 70 & 0.59 & 0.09113 & 0.068625 & 0.02546 & 0.021853 & 0.00203 & 0.000262 & 0.0006 & 0.0001804 & 0.15 & 13 & 1.7 & 26 & 22 & 1449 & 1091 & 13 & 1.7 \\
\hline Zircon_16_026 & 63 & 43 & 0.73 & 0.10996 & 0.038974 & 0.03463 & 0.014435 & 0.00228 & 0.000203 & 0.00066 & $6.14348 \mathrm{E}-05$ & 0.21 & 14.7 & 1.3 & 35 & 15 & 1799 & 638 & 14.7 & 1.3 \\
\hline Zircon_19_029 & 119 & 71 & 0.63 & 0.09098 & 0.030172 & 0.02593 & 0.010163 & 0.00207 & 0.000144 & 0.00061 & $4.18191 \mathrm{E}-05$ & 0.18 & 13.3 & 0.9 & 26 & 10 & 1446 & 480 & 13.3 & 0.9 \\
\hline Zircon_20_030 & 125 & 68 & 0.58 & 0.09166 & 0.026274 & 0.02681 & 0.009447 & 0.00212 & 0.000125 & 0.00063 & $4.19376 \mathrm{E}-05$ & 0.17 & 13.7 & 0.8 & 27 & 10 & 1460 & 419 & 13.7 & 0.8 \\
\hline Zircon_22_033 & 137 & 69 & 0.54 & 0.06973 & 0.02067 & 0.02069 & 0.007362 & 0.00215 & 0.000106 & 0.00065 & $4.20595 \mathrm{E}-05$ & 0.14 & 13.9 & 0.7 & 21 & 7 & 921 & 273 & 13.9 & 0.7 \\
\hline Zircon_04_011 & 121 & 59 & 0.52 & 0.06742 & 0.039845 & 0.01939 & 0.012734 & 0.00209 & 0.000203 & 0.00064 & 0.000180455 & 0.15 & 13.4 & 1.3 & 19 & 12 & 851 & 503 & 13.4 & 1.3 \\
\hline
\end{tabular}

CORRECTED RATIOS

CORRECTED AGES (Ma)

\begin{tabular}{|c|c|c|c|c|c|c|c|c|c|c|c|c|c|c|c|c|c|c|c|c|}
\hline Spot Name & $\mathbf{U}(\mathbf{p p m})^{\S}$ & Th (ppm) $)^{\S}$ & $\mathbf{T h} / \mathbf{U}$ & $207 \mathrm{~Pb} / 206 \mathrm{~Pb}$ & $\pm 2 \sigma^{*}$ & $207 \mathrm{~Pb} / 235 \mathrm{U}$ & $\pm 2 \sigma^{*}$ & $206 \mathrm{~Pb} / 238 \mathrm{U}$ & $\pm 2 \sigma^{*}$ & $208 \mathrm{~Pb} / 232 \mathrm{Th}$ & $\pm 2 \sigma^{*}$ & Rho & $206 \mathrm{~Pb} / 238 \mathrm{U}$ & $\pm 2 \sigma$ & $207 \mathrm{~Pb} / 235 \mathrm{U}$ & $\pm 2 \sigma$ & $207 \mathrm{~Pb} / 206 \mathrm{~Pb}$ & $\pm 2 \sigma$ & Ia) & \\
\hline \multicolumn{21}{|l|}{ Sample: Sin 39} \\
\hline Z_01_SIN39 & 2296 & 4938 & 2.15 & 0.0525 & 0.0026 & 0.0244 & 0.0015 & 0.003401 & 0.000075 & 0.001039 & $5.4 \mathrm{E}-05$ & 0.36 & 21.89 & 0.5 & 24.4 & 1.5 & 324 & 56 & 21.89 & 0.48 \\
\hline Zircon_02 & 1987 & 1840 & 0.93 & 0.0525 & 0.0036 & 0.0249 & 0.0024 & 0.003432 & 0.000083 & 0.001098 & $6.9 \mathrm{E}-05$ & 0.35 & 22.08 & 0.5 & 25 & 2.3 & 330 & 110 & 22.08 & 0.53 \\
\hline Zircon_03 & 1409 & 3430 & 2.43 & 0.0589 & 0.0033 & 0.0281 & 0.0019 & 0.00349 & 0.000087 & 0.001094 & $5.8 \mathrm{E}-05$ & 0.37 & 22.46 & 0.6 & 28.1 & 1.7 & 563 & 74 & 22.46 & 0.56 \\
\hline Zircon_06 & 1361 & 1960 & 1.44 & 0.0627 & 0.0059 & 0.0288 & 0.0043 & 0.003364 & 0.000092 & 0.001176 & 7.1E-05 & 0.18 & 21.65 & 0.6 & 28.8 & 4.2 & 700 & 120 & 21.65 & 0.59 \\
\hline Zircon_07 & 2813 & 4404 & 1.57 & 0.0598 & 0.0032 & 0.0278 & 0.0016 & 0.00334 & 0.00007 & 0.001118 & $5.7 \mathrm{E}-05$ & 0.36 & 21.5 & 0.5 & 27.8 & 1.6 & 600 & 100 & 21.5 & 0.45 \\
\hline Zircon_08 & 2982 & 4327 & 1.45 & 0.0509 & 0.0037 & 0.0227 & 0.0019 & 0.003279 & 0.000067 & 0.001064 & $5.9 \mathrm{E}-05$ & 0.24 & 21.1 & 0.4 & 22.8 & 1.8 & 240 & 120 & 21.1 & 0.43 \\
\hline Zircon_10 & 2230 & 4825 & 2.16 & 0.0474 & 0.0026 & 0.022 & 0.0014 & 0.003349 & 0.000078 & 0.001059 & $5.5 \mathrm{E}-05$ & 0.07 & 21.55 & 0.5 & 22 & 1.3 & 133 & 82 & 21.55 & 0.5 \\
\hline Zircon_11 & 2470 & 4405 & 1.78 & 0.0571 & 0.0029 & 0.0252 & 0.0016 & 0.00327 & 0.000075 & 0.001036 & $5.5 \mathrm{E}-05$ & 0.39 & 21.04 & 0.5 & 25.3 & 1.6 & 459 & 76 & 21.04 & 0.48 \\
\hline Zircon_12 & 4419 & 8919 & 2.02 & 0.0483 & 0.002 & 0.0219 & 0.0012 & 0.003339 & 0.000065 & 0.00103 & $5.2 \mathrm{E}-05$ & 0.23 & 21.49 & 0.4 & 22 & 1.2 & 140 & 50 & 21.49 & 0.42 \\
\hline Zircon_16 & 2137 & 2341 & 1.1 & 0.0502 & 0.0029 & 0.0227 & 0.0018 & 0.003323 & 0.000084 & 0.001102 & $6.2 \mathrm{E}-05$ & 0.15 & 21.38 & 0.5 & 22.8 & 1.8 & 188 & 95 & 21.38 & 0.54 \\
\hline Zircon_17 & 1778 & 4107 & 2.31 & 0.0486 & 0.0031 & 0.0229 & 0.0017 & 0.003425 & 0.000083 & 0.001092 & $5.7 \mathrm{E}-05$ & 0.44 & 22.04 & 0.5 & 23 & 1.6 & 172 & 81 & 22.04 & 0.53 \\
\hline Zircon_18 & 2590 & 3269 & 1.26 & 0.0504 & 0.0025 & 0.0231 & 0.0014 & 0.003327 & 0.000082 & 0.001064 & $5.5 \mathrm{E}-05$ & 0.47 & 21.41 & 0.5 & 23.1 & 1.4 & 244 & 59 & 21.41 & 0.52 \\
\hline Zircon_20 & 994 & 1309 & 1.32 & 0.0508 & 0.0032 & 0.0239 & 0.0017 & 0.003397 & 0.000084 & 0.001064 & $6.2 \mathrm{E}-05$ & 0.27 & 21.86 & 0.5 & 24 & 1.7 & 253 & 58 & 21.86 & 0.54 \\
\hline Zircon_22 & 2998 & 5080 & 1.69 & 0.0481 & 0.0025 & 0.0219 & 0.0014 & 0.003342 & 0.000066 & 0.001102 & $5.5 \mathrm{E}-05$ & 0.31 & 21.51 & 0.4 & 22 & 1.3 & 138 & 69 & 21.51 & 0.42 \\
\hline Zircon_23 & 2299 & 4393 & 1.91 & 0.0554 & 0.0025 & 0.026 & 0.0015 & 0.00335 & 0.000075 & 0.001043 & $5.3 \mathrm{E}-05$ & 0.59 & 21.56 & 0.5 & 26.1 & 1.5 & 423 & 70 & 21.56 & 0.48 \\
\hline Zircon_24 & 1947 & 2463 & 1.27 & 0.0499 & 0.0026 & 0.0235 & 0.0015 & 0.003373 & 0.000067 & 0.00109 & $5.7 \mathrm{E}-05$ & 0.23 & 21.71 & 0.4 & 23.6 & 1.5 & 225 & 64 & 21.71 & 0.43 \\
\hline Zircon_27 & 1610 & 2300 & 1.43 & 0.0546 & 0.0025 & 0.0252 & 0.0013 & 0.003376 & 0.000073 & 0.001059 & $5.4 \mathrm{E}-05$ & 0.64 & 21.73 & 0.5 & 25.3 & 1.3 & 366 & 68 & 21.73 & 0.47 \\
\hline Zircon_28 & 2368 & 3313 & 1.4 & 0.051 & 0.0026 & 0.0237 & 0.0015 & 0.003338 & 0.000076 & 0.001045 & $5.5 \mathrm{E}-05$ & 0.21 & 21.48 & 0.5 & 23.7 & 1.5 & 335 & 65 & 21.48 & 0.49 \\
\hline Zircon_31 & 720 & 914 & 1.27 & 0.0562 & 0.0029 & 0.0265 & 0.0021 & 0.00345 & 0.00024 & 0.001099 & $5.9 \mathrm{E}-05$ & 0.24 & 22.2 & 1.6 & 26.5 & 2.1 & 377 & 59 & 22.2 & 1.6 \\
\hline Zircon_32 & 2172 & 2426 & 1.12 & 0.0517 & 0.0026 & 0.0234 & 0.0015 & 0.003305 & 0.000076 & 0.001092 & $5.8 \mathrm{E}-05$ & 0.33 & 21.27 & 0.5 & 23.5 & 1.4 & 252 & 69 & 21.27 & 0.49 \\
\hline Zircon_33 & 571 & 944 & 1.65 & 0.0549 & 0.0069 & 0.0266 & 0.0043 & 0.0035 & 0.00011 & 0.001167 & $7.7 \mathrm{E}-05$ & 0.75 & 22.53 & 0.7 & 26.6 & 4.2 & 440 & 160 & 22.53 & 0.68 \\
\hline Zircon_34 & 1870 & 2815 & 1.51 & 0.05 & 0.0024 & 0.0231 & 0.0015 & 0.003332 & 0.000076 & 0.001105 & $6.3 \mathrm{E}-05$ & 0.35 & 21.44 & 0.5 & 23.2 & 1.5 & 209 & 66 & 21.44 & 0.49 \\
\hline Zircon_35 & 2495 & 4301 & 1.72 & 0.0513 & 0.0024 & 0.0237 & 0.0013 & 0.00337 & 0.000072 & 0.00105 & $5.3 \mathrm{E}-05$ & 0.39 & 21.69 & 0.5 & 23.8 & 1.2 & 280 & 51 & 21.69 & 0.46 \\
\hline Zircon_36 & 4248 & 9091 & 2.14 & 0.0495 & 0.0021 & 0.0228 & 0.0012 & 0.003337 & 0.000064 & 0.001034 & $5.2 \mathrm{E}-05$ & 0.22 & 21.48 & 0.4 & 22.9 & 1.2 & 194 & 50 & 21.48 & 0.41 \\
\hline Zircon_37 & 1090 & 1912 & 1.75 & 0.0575 & 0.0052 & 0.0266 & 0.004 & 0.003501 & 0.000098 & 0.001181 & $7.1 \mathrm{E}-05$ & 0.3 & 22.53 & 0.6 & 26.6 & 3.9 & 530 & 140 & 22.53 & 0.63 \\
\hline
\end{tabular}


CORRECTED RATIOS ${ }^{\dagger, *}$

CORRECTED AGES (Ma)

\begin{tabular}{|c|c|c|c|c|c|c|c|c|c|c|c|c|c|c|c|c|c|c|c|c|}
\hline \multirow{2}{*}{ Spot Name } & \multirow[b]{2}{*}{$\mathbf{U}(\mathbf{p p m})^{\S}$} & \multirow[b]{2}{*}{ Th (ppm) $)^{\S}$} & \multirow[b]{2}{*}{$\mathrm{Th} / \mathrm{U}$} & & & & & & & & & & & & & & & & \multirow{2}{*}{$\begin{array}{l}\text { Best age } \\
\text { (Ma) }\end{array}$} & \multirow{2}{*}{ $\pm 2 \sigma$} \\
\hline & & & & $207 \mathrm{~Pb} / 206 \mathrm{~Pb}$ & $\pm 2 \sigma^{*}$ & $207 \mathrm{~Pb} / 235 \mathrm{U}$ & $\pm 2 \sigma^{*}$ & $206 \mathrm{~Pb} / 238 \mathrm{U}$ & $\pm 2 \sigma^{*}$ & $208 \mathrm{~Pb} / 232 \mathrm{Th}$ & $\pm 2 \sigma^{*}$ & Rho & $206 \mathrm{~Pb} / 238 \mathrm{U}$ & $\pm 2 \sigma$ & $207 \mathrm{~Pb} / 235 \mathrm{U}$ & $\pm 2 \sigma$ & $207 \mathrm{~Pb} / 206 \mathrm{~Pb}$ & $\pm 2 \sigma$ & & \\
\hline Sample: Sin 05 & & & & & & & & & & & & & & & & & & & & \\
\hline Z_01_SIN-05 & 69 & 31 & 0.45 & 0.089 & 0.013 & 0.0625 & 0.0094 & 0.00523 & 0.00031 & 0.00192 & 0.0002 & 0.24732 & 33.7 & 2 & 61.3 & 9 & 1400 & 170 & 33.7 & 2 \\
\hline Zircon_02 & 150 & 95 & 0.63 & 0.088 & 0.0064 & 0.0611 & 0.0055 & 0.00511 & 0.00026 & 0.00227 & 0.00015 & 0.565238 & 32.9 & 1.7 & 60.2 & 5.3 & 1415 & 96 & 32.9 & 1.7 \\
\hline Zircon_03 & 260 & 118 & 0.45 & 0.061 & 0.02 & 0.041 & 0.018 & 0.00481 & 0.00032 & 0.00199 & 0.00084 & 0.082681 & 30.9 & 2 & 41 & 17 & 720 & 300 & 30.9 & 2 \\
\hline Zircon_04 & 363 & 207 & 0.57 & 0.059 & 0.0037 & 0.0389 & 0.0031 & 0.00482 & 0.00024 & 0.00162 & 0.00011 & 0.624816 & 31 & 1.5 & 38.7 & 3.1 & 626 & 66 & 31 & 1.5 \\
\hline Zircon_05 & 361 & 408 & 1.13 & 0.047 & 0.052 & 0.031 & 0.071 & 0.00481 & 0.00067 & 0.0016 & 0.0018 & 0.15554 & 30.9 & 4.3 & 31 & 56 & 360 & 470 & 30.9 & 4.3 \\
\hline Zircon_06 & 47 & 39 & 0.83 & 0.094 & 0.015 & 0.073 & 0.011 & 0.00531 & 0.00033 & 0.00201 & 0.0002 & 0.412429 & 34.2 & 2.1 & 70 & 11 & 1660 & 210 & 34.2 & 2.1 \\
\hline Zircon_07 & 161 & 79 & 0.49 & 0.057 & 0.0069 & 0.0399 & 0.0058 & 0.0054 & 0.00028 & 0.00176 & 0.00022 & 0.17102 & 34.7 & 1.8 & 39.6 & 5.6 & 600 & 150 & 34.7 & 1.8 \\
\hline Zircon_08 & 179 & 85 & 0.47 & 0.0875 & 0.0069 & 0.059 & 0.0055 & 0.00517 & 0.00028 & 0.00241 & 0.00017 & 0.580974 & 33.2 & 1.8 & 58.2 & 5.2 & 1310 & 100 & 33.2 & 1.8 \\
\hline Zircon_09 & 686 & 286 & 0.42 & 0.051 & 0.0032 & 0.0339 & 0.0028 & 0.00481 & 0.00023 & 0.001496 & 0.000091 & 0.578928 & 30.9 & 1.5 & 33.9 & 2.7 & 356 & 86 & 30.9 & 1.5 \\
\hline Zircon_10 & 140 & 70 & 0.50 & 0.0486 & 0.0064 & 0.0322 & 0.0047 & 0.00476 & 0.00027 & 0.00148 & 0.00013 & 0.388611 & 30.6 & 1.7 & 32.2 & 4.6 & 580 & 140 & 30.6 & 1.7 \\
\hline Zircon_11 & 828 & 702 & 0.85 & 0.0495 & 0.0028 & 0.0333 & 0.0029 & 0.00493 & 0.00025 & 0.00159 & 0.000096 & 0.58229 & 31.7 & 1.6 & 33.2 & 2.8 & 346 & 54 & 31.7 & 1.6 \\
\hline Zircon_12 & 275 & 120 & 0.44 & 0.0664 & 0.0064 & 0.0441 & 0.006 & 0.00473 & 0.00029 & 0.00193 & 0.00018 & 0.450634 & 30.4 & 1.9 & 43.8 & 5.8 & 810 & 140 & 30.4 & 1.9 \\
\hline Zircon_13 & 96 & 63 & 0.66 & 0.0786 & 0.0098 & 0.054 & 0.0076 & 0.00509 & 0.0003 & 0.00182 & 0.00015 & 0.418778 & 32.7 & 1.9 & 53.2 & 7.3 & 1260 & 120 & 32.7 & 1.9 \\
\hline Zircon_14 & 193 & 129 & 0.67 & 0.0731 & 0.0078 & 0.0479 & 0.0061 & 0.00479 & 0.00028 & 0.0015 & 0.00014 & 0.459016 & 30.8 & 1.8 & 47.5 & 5.9 & 1020 & 160 & 30.8 & 1.8 \\
\hline Zircon_15 & 115 & 88 & 0.77 & 0.095 & 0.031 & 0.067 & 0.036 & 0.00511 & 0.00045 & 0.00196 & 0.00091 & 0.163894 & 32.8 & 2.9 & 66 & 30 & 1530 & 240 & 32.8 & 2.9 \\
\hline Zircon_17 & 218 & 100 & 0.46 & 0.0648 & 0.0053 & 0.0419 & 0.0045 & 0.00473 & 0.00025 & 0.0015 & 0.00012 & 0.492131 & 30.4 & 1.6 & 41.7 & 4.4 & 869 & 90 & 30.4 & 1.6 \\
\hline Zircon_18 & 284 & 111 & 0.39 & 0.0745 & 0.005 & 0.0485 & 0.0041 & 0.00481 & 0.00025 & 0.00194 & 0.00014 & 0.614827 & 30.9 & 1.6 & 48 & 4 & 1010 & 100 & 30.9 & 1.6 \\
\hline Zircon_19 & 35 & 20 & 0.57 & 0.106 & 0.055 & 0.073 & 0.068 & 0.00536 & 0.00064 & 0.002 & 0.0014 & 0.50941 & 34.5 & 4.1 & 71 & 54 & 1650 & 420 & 34.5 & 4.1 \\
\hline Zircon_20 & 91 & 43 & 0.47 & 0.064 & 0.017 & 0.043 & 0.016 & 0.00488 & 0.00033 & 0.00171 & 0.00026 & 0.41426 & 31.4 & 2.1 & 42 & 15 & 890 & 280 & 31.4 & 2.1 \\
\hline Zircon_21 & 104 & 24 & 0.23 & 0.069 & 0.0065 & 0.0464 & 0.0053 & 0.00493 & 0.00028 & 0.00166 & 0.00017 & 0.063001 & 31.7 & 1.8 & 46 & 5.2 & 996 & 98 & 31.7 & 1.8 \\
\hline Zircon_22 & 90 & 31 & 0.34 & 0.0674 & 0.0073 & 0.0455 & 0.0059 & 0.00488 & 0.00029 & 0.00163 & 0.00016 & 0.088277 & 31.4 & 1.9 & 45.1 & 5.8 & 940 & 120 & 31.4 & 1.9 \\
\hline Zircon_23 & 120 & 52 & 0.43 & 0.092 & 0.019 & 0.063 & 0.019 & 0.00503 & 0.00032 & 0.00202 & 0.00048 & 0.210945 & 32.4 & 2.1 & 62 & 17 & 1480 & 180 & 32.4 & 2.1 \\
\hline Zircon_24 & 240 & 92 & 0.38 & 0.0618 & 0.0046 & 0.0388 & 0.0039 & 0.00482 & 0.00024 & 0.00156 & 0.00013 & 0.25625 & 31 & 1.6 & 38.7 & 3.8 & 657 & 72 & 31 & 1.6 \\
\hline Zircon_25 & 154 & 54 & 0.35 & 0.106 & 0.035 & 0.074 & 0.039 & 0.00545 & 0.00042 & 0.0028 & 0.0011 & 0.146224 & 35 & 2.7 & 73 & 34 & 1630 & 260 & 35 & 2.7 \\
\hline Zircon_26 & 147 & 73 & 0.50 & 0.0568 & 0.0076 & 0.0386 & 0.0055 & 0.00494 & 0.00025 & 0.00156 & 0.00012 & 0.355171 & 31.8 & 1.6 & 38.4 & 5.3 & 630 & 130 & 31.8 & 1.6 \\
\hline Zircon_27 & 87 & 108 & 1.24 & 0.123 & 0.04 & 0.087 & 0.093 & 0.00491 & 0.00069 & 0.0022 & 0.00045 & 0.34689 & 31.5 & 4.4 & 85 & 57 & 2000 & 120 & 31.5 & 4.4 \\
\hline Zircon_28 & 182 & 105 & 0.58 & 0.0609 & 0.0041 & 0.041 & 0.004 & 0.00485 & 0.00025 & 0.00182 & 0.00013 & 0.24693 & 31.2 & 1.6 & 40.8 & 3.9 & 644 & 85 & 31.2 & 1.6 \\
\hline Zircon_29 & 611 & 401 & 0.66 & 0.0607 & 0.0027 & 0.0399 & 0.0029 & 0.00471 & 0.00023 & 0.00165 & 0.0001 & 0.027431 & 30.3 & 1.5 & 39.7 & 2.9 & 640 & 56 & 30.3 & 1.5 \\
\hline Zircon_30 & 176 & 107 & 0.61 & 0.059 & 0.0057 & 0.0407 & 0.0048 & 0.00496 & 0.00027 & 0.00163 & 0.00013 & 0.461568 & 31.9 & 1.8 & 40.4 & 4.7 & 652 & 73 & 31.9 & 1.8 \\
\hline Zircon_31 & 277 & 191 & 0.69 & 0.0499 & 0.0079 & 0.0326 & 0.0064 & 0.00477 & 0.00025 & 0.00165 & 0.00015 & 0.266968 & 30.7 & 1.6 & 32.6 & 6.2 & 440 & 160 & 30.7 & 1.6 \\
\hline Zircon_32 & 949 & 575 & 0.61 & 0.0581 & 0.0035 & 0.0383 & 0.0034 & 0.00476 & 0.00023 & 0.00165 & 0.00012 & 0.045109 & 30.6 & 1.5 & 38.2 & 3.3 & 486 & 80 & 30.6 & 1.5 \\
\hline Zircon_33 & 7030 & 2905 & 0.41 & 0.0587 & 0.0035 & 0.0451 & 0.0042 & 0.00556 & 0.00027 & 0.00234 & 0.00022 & 0.6066 & 35.7 & 1.7 & 44.8 & 4.1 & 550 & 120 & 35.7 & 1.7 \\
\hline Zircon_34 & 201 & 127 & 0.63 & 0.064 & 0.011 & 0.0434 & 0.0083 & 0.00485 & 0.00029 & 0.00167 & 0.0002 & 0.043091 & 31.2 & 1.8 & 43 & 8 & 1150 & 150 & 31.2 & 1.8 \\
\hline Zircon_35 & 131 & 109 & 0.83 & 0.079 & 0.032 & 0.052 & 0.033 & 0.00487 & 0.00047 & 0.0016 & 0.0012 & 0.24758 & 31.3 & 3 & 52 & 28 & 1330 & 310 & 31.3 & 3 \\
\hline
\end{tabular}


\$: U and Th concentrations are calculated employing the NIST 610 glass as external standard.

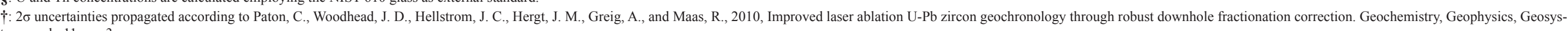

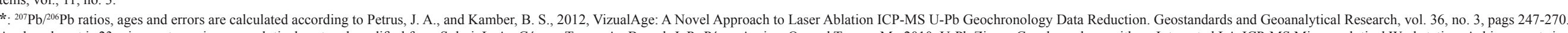

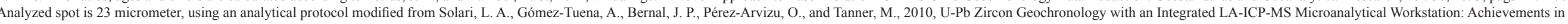
Precision and Accuracy. Geostandards and Geoanalytical Research, vol. 34, no. 1, pags 5-18. Data measured employing a Thermo X-series quadrupole ICPMS coupled to a Resonetics, Resolution M050 excimer laser workstation. N.D.: Not determined. 


\section{Discussion of ${ }^{40} \mathrm{Ar}-{ }^{39} \mathrm{Ar}$ dating experiments}

A brief discussion of the ${ }^{40} \mathrm{Ar}^{39}{ }^{39} \mathrm{Ar}$ results is given here for each simple, providing basis for the selection of the preferred age reported in the text (i.e. isochron or plateau). The samples are reported in the same order as in Table 1 (oldest to newest).

\section{ES 10 basaltic andesite}

Two laser step-heating experiments were performed on plagioclase with 6 and 8 fractions collected. Well defined plateaux are displayed in both experiments. The plateau age of $34.17 \pm 0.31 \mathrm{Ma}$ (MSWD $=0.53$ ) was calculated with 5 consecutive fractions representing $86.66 \%$ of the ${ }^{39} \mathrm{Ar}$ released. The preferred age for this simple is the isochron age $33.82 \pm 0.28$ Ma using the combined fractions of the two experiments $(\mathrm{MSWD}=1.1$ for $\mathrm{n}=14)$. The isochron age is slightly younger than the plateau age because the $\left({ }^{40} \mathrm{Ar}{ }^{36} \mathrm{Ar}\right)_{\mathrm{i}}=298 \pm 2$ is slightly above the atmospheric value; for this reason the isochron age was selected as the best age estimate for sample ES 10.

\section{HUA 1 crystal rich ignimbrite}

Two laser step-heating experiments were performed on biotite. The two experiments yielded reproducible results. The plateau age of $26.48 \pm 0.15 \mathrm{Ma}$ was obtained from the weighted mean of the last four fractions of the second experiment, which gave a MSWD $=0.18$ for $96.61 \%$ of the ${ }^{39} \mathrm{Ar}$ released. The isochron calculated with the fractions representing the bulk of the ${ }^{39} \mathrm{Ar}$ from both experiments $\left(92.19 \%\right.$ and $86.83 \%$ respectively) yields an age of $26.51 \pm 0.69 \mathrm{Ma}$ with a $\left({ }^{40} \mathrm{Ar}{ }^{36} \mathrm{Ar}\right)_{\mathrm{i}}=$ $295 \pm 69$, and an MSWD $=0.32$ for $\mathrm{n}=5$, in very good agreement with the plateau age. We chose the plateau age of $26.48 \pm 0.15 \mathrm{Ma}$ as our best estimate for the age of this sample.

\section{TS 16 basalt}

A plagioclase concentrate was laser step-heated. Six fractions were collected. Because this plagioclase is calcium-rich but has very low $\mathrm{K}$ content we obtained a low precision results. Applying 12 watts of laser power over $50 \%$ of the ${ }^{39} \mathrm{Ar}$ was released in the last fraction collected. The individual ages for the 6 fractions define a plateau age of $24.38 \pm 0.75 \mathrm{Ma}(\mathrm{MSWD}=0.54)$. The plateau age is practically identical to the isochron age of $24.43 \pm 0.87 \mathrm{Ma}$ obtained using all the data points.

\section{HUA 2 lithic ignimbrite}

Two laser step-heating experiments were performed on a biotite concentrate. No age information could be obtained for the last fraction of the first experiment because the signal of mass 36 was not measured correctly. The data of this experiment are presented in the Electronic Supplementary Material, only for illustrative purpose. For the second experiment a broad, well defined plateau age of $22.99 \pm 0.14 \mathrm{Ma}$ $\left(\mathrm{MSWD}=0.63\right.$ ), was obtained for $94 \%$ of the ${ }^{39} \mathrm{Ar}$ released in five steps. Combining the six fractions, released with more than 1.5 Watts of laser power from both experiments, an isochron age of $23.19 \pm$ $0.27 \mathrm{Ma}$ was obtained. The straight line is well defined with $\mathrm{MSWD}=0.35$, but due to the point distribution, the y-intercept is poorly constrained with $\left({ }^{40} \mathrm{Ar}{ }^{36} \mathrm{Ar}\right)_{\mathrm{i}}=282 \pm 21$. The isochron and plateau ages are statistically indistinguishable but we favour the more precise plateau age of $22.99 \pm$ $0.14 \mathrm{Ma}$ for this sample. 


\section{ESC 7 basaltic andesite}

Two step-heating experiments were performed using the temperature controlled Ta-furnace on $\sim 0.2$ and $\sim 0.3$ gr. of plagioclase. The two experiments yielded reproducible results. Because the plagioclase had low K content the ages obtained were not very precise. For the second experiment a well defined plateau age of $22.39 \pm 2.56 \mathrm{Ma}(\mathrm{MSWD}<0.01$ for $\mathrm{n}=5)$ was obtained. This was calculated with the fractions released between 700 and $1350^{\circ} \mathrm{C}$, which represent $97.2 \%$ of the ${ }^{39} \mathrm{Ar}$ released. The plateau age is statistically indistinguishable from the isochron age of $22.35 \pm 2.73 \mathrm{Ma}$ (MSWD $=0.11$ for $\mathrm{n}=$ 11), obtained combining the fractions of the two experiments. We favour the plateau age of $22.39 \pm$ $2.56 \mathrm{Ma}$ because os its slightly lower error.

\section{MdCH10 dacite}

A plagioclase concentrate was laser step-heated. The two experiments performed yielded reproducible results. Because the plagioclase had very low K content, the experiments were not detailed. Due to analytical dificulties, on three of the low temperature fractions, mass 36 was not measured correctly; no age information could be obtained from them. However, since the bulk of the ${ }^{39} \mathrm{Ar}$ was released at high temperature, the low temperature fractions were not significant. With the fractions measured correctly a well-defined plateau age of $22.15 \pm 0.93 \mathrm{Ma}(\mathrm{MSWD}=0.59$ for $\mathrm{n}=3$ ), was obtained for $88.50 \%$ of the ${ }^{39} \mathrm{Ar}$ released. The isochron age of $22.51 \pm 0.90 \mathrm{Ma}$, calculated with the combined fractions of the two experiments $(\mathrm{MSWD}=0.73$ for $n=6)$ is the preferred age for dacite MdCH10.

\section{ORO 2 ignimbrite}

A laser step-heating experiment was performed on a biotite concentrate. The biotite displays a flat age spectrum with a wide plateau of $20.88 \pm 0.12 \mathrm{Ma}\left(\mathrm{MSWD}=0.85\right.$ for $\mathrm{n}=6$ ) for $99.73 \%$ of the ${ }^{39} \mathrm{Ar}$ released. In the ${ }^{36} \mathrm{Ar} /{ }^{40} \mathrm{Ar}$ versus ${ }^{39} \mathrm{Ar} /{ }^{40} \mathrm{Ar}$ correlation diagram, the bulk of the ${ }^{39} \mathrm{Ar}$ released in the last four fractions define a straight line $(\mathrm{MSWD}=0.19)$ with a $\left({ }^{40} \mathrm{Ar} /{ }^{36} \mathrm{Ar}\right)_{\mathrm{i}}=325 \pm 59$, suggesting the presence of some excess argon. Therefore we chose the isochron age of $20.74 \pm 0.44 \mathrm{Ma}$ as our best estimate for this sample.

\section{MdCH 06 rhyolite}

Two laser step-heating experiments were performed on a plagioclase concentrate. During the first experiment, more than $50 \%$ of the ${ }^{39}$ Ar was released in one fraction. To insure that this result was realiable, a different heating schedule was chosen for the second experiment, in which we obtained a wide plateau age of $20.54 \pm 0.79 \mathrm{Ma}(\mathrm{MSWD}=0.45$ for $\mathrm{n}=4)$ representing $78.72 \%$ of the ${ }^{39} \mathrm{Ar}$ released. The age spectra display a small saddle suggesting the presence of excess argon. The combined data of the two experiments constrain an isochron age of $20.25 \pm 0.64 \mathrm{Ma}(\mathrm{MSWD}=0.98$ for $\mathrm{n}=22)$, with $\left({ }^{40} \mathrm{Ar} /{ }^{36} \mathrm{Ar}\right)_{\mathrm{i}}=304 \pm 2$ confirming the presence of excess argon. For this reason, we favour the isochron age of $20.25 \pm 0.64 \mathrm{Ma}$ for this sample.

\section{HUA 5 ash flow tuff}

Two laser step-heating experiments were performed on a plagioclase concentrate with reproducible results. In the two experiments, the bulk of the ${ }^{39} \mathrm{Ar}$ was released in the last fraction. The weighted mean of two fractions from the second experiment yielded an age of $18.99 \pm 0.27 \mathrm{Ma}(\mathrm{MSWD}=0.09$ for $91.75 \%$ of ${ }^{39} \mathrm{Ar}$ released), in complete agreement with the isochron age of $18.98 \pm 0.30 \mathrm{Ma}$ $(\mathrm{MSWD}=1.57$ for $\mathrm{n}=5$ ) obtained with the combined the data of the two experiments. The preferred age is the isochron age because combined data from the two experiments. 


\section{MdCH 09 olivine basalt}

A plagioclase concentrate from this sample was laser step-heated obtaining reproducible results. The plagioclase analyzed is $\mathrm{Ca}$-rich, with $\mathrm{Ca} / \mathrm{K} \sim 30$ and a high temperature release of ${ }^{39} \mathrm{Ar}$. For this reason it needed more than 8 watts of laser power to release the bulk of ${ }^{39} \mathrm{Ar}$. From the second experiment, a plateau age of $18.34 \pm 0.39 \mathrm{Ma}$ is defined by the last five fractions, which represent $72.06 \%$ of the ${ }^{39} \mathrm{Ar}$ released. The same age was obtained plotting the data of the two experiments in the ${ }^{36} \mathrm{Ar} /{ }^{40} \mathrm{Ar}$ versus ${ }^{39} \mathrm{Ar} /{ }^{40} \mathrm{Ar}$ correlation diagram. An isochron age of $18.32 \pm 0.40 \mathrm{Ma}$ was calculated with an MSWD $=$ 1.6 for the 11 points fitted. The plateau and isochron ages obtained are statistically indistinguishable but we favour the isochron age as our best estimate because it incorporates the data of the two experiments.

\section{RUIZ 16 rhyolite}

Two laser step-heating experiments were performed on plagioclase concentrate yielding reproducible results and almost flat age spectra. The 7 fractions collected in the second experiment fullfill the criteria to calculate a plateau age. The weigthted mean of their individual ages yields a plateau age of $17.76 \pm 0.12 \mathrm{Ma}(\mathrm{MSWD}=0.5)$. The plateau age is statistically indistinguishable from the isochron age of $17.91 \pm 0.20 \mathrm{Ma}$, obtained with the combined fractions of the two experiments. The isochron age is taken as the best estimate for this rhyolite since it contains the data of the two experiments.

\section{RUIZ 34B rhyolite}

A biotite concentrate was laser step-heated. The experiment yielded a flat age spectra, with a broad plateau defined by seven fractions with $86.74 \%$ of the ${ }^{39} \mathrm{Ar}$ released and an age of $17.82 \pm 0.07 \mathrm{Ma}$. Because the best straight line (MSWD $=0.79$ ) defined by 9 out of the 12 fractions collected yields a $\left({ }^{40} \mathrm{Ar} /{ }^{36} \mathrm{Ar}\right)_{\mathrm{i}}=357 \pm 40$, the isochron age of $17.57 \pm 0.19 \mathrm{Ma}$ is taken as the preferred age for this sample.

\section{PER 12 basaltic andesite}

One gram of groundmass concentrate was step-heated with the temperature controlled Ta-furnace and the argon isotopes were analized with the MS-10 mass spectrometer. The ${ }^{39} \mathrm{Ar}$ was released evenly over the 10 fractions collected between 500 and $1350^{\circ} \mathrm{C}$. Six fractions representing $62.65 \%$ of the ${ }^{39} \mathrm{Ar}$ released fulfill the criteria to calculate a plateau. The weighted mean of the individual ages yields a plateau of $17.78 \pm 0.37 \mathrm{Ma}$. The age spectrum displays a descending staircase pattern, which may be indicative of excess argon. The data were plotted in the ${ }^{36} \mathrm{Ar} /{ }^{40} \mathrm{Ar}$ versus ${ }^{39} \mathrm{Ar} /{ }^{40} \mathrm{Ar}$ correlation diagram, where

7 consecutive fractions representing more than $88 \%$ of the ${ }^{39} \mathrm{Ar}$ released, define a straight line with a yintercept confirming the presence of excess argon with an $\left({ }^{40} \mathrm{Ar} /{ }^{36} \mathrm{Ar}\right)_{\mathrm{i}}=335 \pm 19$. The $\mathrm{x}$-intercept defines an isochron age of $17.42 \pm 0.77 \mathrm{Ma}$, which is our preferred age given that the presence of excess argon was confirmed.

\section{MdCH 05 rhyolite}

For this sample we carried out a laser step heating experiment on groundmass collecting a total of 19 fractions. The first third of the ${ }^{39}$ Ar released yielded an age spectrum with a decreasing staircase pattern. The remaining fractions define a plateau age of $17.41 \pm 0.07 \mathrm{Ma}$ with the ${ }^{39} \mathrm{Ar}$ evenly distributed in 14 fractions. The plateau fractions (identified with letters $f$ to $s$ ) align in the ${ }^{36} \mathrm{Ar} /{ }^{40} \mathrm{Ar}$ versus ${ }^{39} \mathrm{Ar} /{ }^{40} \mathrm{Ar}$ correlation diagram defining an isochron age of $17.52 \pm 0.24$. However the points are 
narrowly distributed close to the x-axis, so that the y-intercept $(292 \pm 9)$ is not well constrained. Because of this we favour the well-defined plateau age of $17.41 \pm 0.07 \mathrm{Ma}$ for this rhyolite.

\section{SIN 25 basalt}

Twelve fractions were collected in the laser step-heating experiment performed on whole rock. The sample displays an almost flat age spectrum with a slight negative slope. Ten consecutive fractions representing more than $98 \%$ of the ${ }^{39}$ Ar released fulfill the criteria to calculate the plateau age of 14.31 $\pm 0.11 \mathrm{Ma}$. However the plateau segment displays variable composition in the ${ }^{37} \mathrm{Ar}_{\mathrm{Ca}}{ }^{39} \mathrm{Ar}_{\mathrm{K}}$ diagram. An iscohron age of $14.01 \pm 0.23 \mathrm{Ma}$ was calculated with the last 9 fractions $(\mathrm{MSWD}=0.82)$. The $\left({ }^{40} \mathrm{Ar} /{ }^{36} \mathrm{Ar}\right)_{\mathrm{i}}=315 \pm 11$, obtained in the correlation diagram indicates excess argon and explains the subtle negative slope displayed in the age spectrum. Therefore our prefered age for this basalt is the isochron age.

\section{SIN 15 basalt}

A groundmass concentrate was laser step-heated. The weighted mean of the individual ages of 7 consecutive fractions representing $94.03 \%$ of the ${ }^{39}$ Ar released yield a $13.84 \pm 0.15$ Ma plateau age. However, the older ages for the fractions released at low temperature suggest the presence of excess argon. This is confirmed by the $\left({ }^{40} \mathrm{Ar} /{ }^{36} \mathrm{Ar}\right)_{\mathrm{i}}=304 \pm 4$ calculated from the y-intercept of the straight line defined with all the data in the ${ }^{36} \mathrm{Ar} /{ }^{40} \mathrm{Ar}$ vs ${ }^{39} \mathrm{Ar} /{ }^{40} \mathrm{Ar}$ correlation diagram. For this reason we favour the isochron age of $13.62 \pm 0.17 \mathrm{Ma}$ for this basalt.

\section{DANA 46a basalt}

This whole rock sample was analyzed in detail. The first set of experiments, from irradiation labeled CIC 41A, consisted of a step-heating experiment with the temperature controlled Ta-furnace and 8 one-step laser fusion experiments. For the step-heating experiment the argon isotopes were measured with the MS-10 mass spectrometer while the argon isotopes of the laser fusion experiments were measured with the VG5400 mass spectrometer. A flat age spectrum was obtained yielding a plateau age of $11.97 \pm 0.30 \mathrm{Ma}$, for $100 \%$ of the ${ }^{39} \mathrm{Ar}$ released in five fractions. The results from the one-step experiments were remarkably similar and yielded statistically undistinguishable ages. The sample was irradiated again and another set of one-step laser fusion runs was performed plus a laser-step-heating experiment. The second group of experiments yielded reproducible results in agreement with the first data set. The weighted mean of all the one-step laser fusions yields an age of 11.59 $\pm 0.31 \mathrm{Ma}$ (MSWD $=0.89$ for $\mathrm{n}=13$ ). The 21 data points from all the experiments are well distributed and aligned in the ${ }^{36} \mathrm{Ar} /{ }^{40} \mathrm{Ar}$ versus ${ }^{39} \mathrm{Ar} /{ }^{40} \mathrm{Ar}$ correlation diagram. The well defined straight line (MSWD $\left.=0.50\right)$ yields a $\left({ }^{40} \mathrm{Ar} /{ }^{36} \mathrm{Ar}\right)_{\mathrm{i}}=291 \pm 3$ and an isochron age of $11.96 \pm 0.26 \mathrm{Ma}$. This group of experiments is a good example of the reproducibility of results produced at CICESE's Geochronology Laboratory applying different heating techniques and two mass spectrometers. We favour the $11.96 \pm 0.26 \mathrm{Ma}$ isochron age as the best estimate for basalt DANA 46a, because it incorporates the data from all the experiments performed with this sample.

\section{ROCA 3J 5 lithic tuff}

Two laser step-heating experiments were performed on feldspar concentrate yielding reproducible and flat age spectra. The six fractions collected in the second experiment yield individual ages statistically indistinguishable within $1 \sigma$ errors, defining a plateau age of $11.70 \pm 0.07 \mathrm{Ma}$. The same age was obtained combining the data from the two experiments, in the ${ }^{36} \mathrm{Ar} /{ }^{40} \mathrm{Ar}$ versus ${ }^{39} \mathrm{Ar} /{ }^{40} \mathrm{Ar}$ correlation diagram: $11.69 \pm 0.08 \mathrm{Ma}$. We chose the more precise plateau age as the best estimate for this sample. 


\section{LM 2 dacite}

Two laser step-heating experiments were performed on a groundmass concentrate. The pronounced and reproducible saddle shaped age spectra obtained suggests the presence of excess argon. With the purpose of improving the results, a different heating schedule was selected in the second experiment. The weighted mean of the individual ages of the fractions that define the base of the saddle yield an age of $13.38 \pm 0.61 \mathrm{Ma}$. This U-shaped age spectra is also displayed in the ${ }^{37} \mathrm{Ar}_{\mathrm{Ca}}{ }^{39} \mathrm{Ar}_{\mathrm{K}}$ diagram indicating a homogeneous composition in the calcium and potasium composition for the fractions collected at intermediate laser power, that is between 0.5 and 4 Watts. The data of the two experiments were plotted in the ${ }^{36} \mathrm{Ar} /{ }^{40} \mathrm{Ar}$ versus ${ }^{39} \mathrm{Ar} /{ }^{40} \mathrm{Ar}$ correlation diagram. The 8 fractions with ${ }^{37} \mathrm{Ar}_{\mathrm{Ca}} /{ }^{39} \mathrm{Ar}_{\mathrm{K}}$ values $\leq 0.6$ define a straight line with $\left({ }^{40} \mathrm{Ar} /{ }^{36} \mathrm{Ar}\right)_{\mathrm{i}}=400 \pm 10$, corroborating the presence of excess argon. Therefore we prefer the isochron age of $11.34 \pm 0.79 \mathrm{Ma}$ as the best age estimate for this dacite.

\section{ROCA 24J 33 microdiorite}

A hornblende concentrate was laser step-heated. The sample displays a perturbed age spectrum with a pronounced saddle shape, which suggests the presence of excess argon. The weighted mean of the last four fractions yields an age of $12.14 \pm 0.34 \mathrm{Ma}$. These fractions represent $66.40 \%$ of the ${ }^{39} \mathrm{Ar}$ released and have variable values of ${ }^{37} \mathrm{Ar}_{\mathrm{Ca}}{ }^{39} \mathrm{Ar}_{\mathrm{K}}$, suggesting inhomogeneous composition for the hornblende. The data are very well distributed and aligned in the ${ }^{36} \mathrm{Ar} /{ }^{40} \mathrm{Ar}$ versus ${ }^{39} \mathrm{Ar} /{ }^{40} \mathrm{Ar}$ correlation diagram. The straight line defined by the data intercepts the y-axis at $\left({ }^{40} \mathrm{Ar}{ }^{36} \mathrm{Ar}\right)_{\mathrm{i}}=313 \pm 4$, confirming the presence of excess argon. For this reason, the isochron age of $11.29 \pm 0.37 \mathrm{Ma}$ is our preferred age for this sample.

\section{PER 8 basaltic andesite}

One laser step-heating experiment was performed on a groundmass concentrate. Only five fractions were collected, with the bulk of the ${ }^{39} \mathrm{Ar}$ released in the last three fractions. The weighted mean of the individual ages of these three fractions yielded a $10.94 \pm 0.23 \mathrm{Ma}$ age. The data aligns in the ${ }^{36} \mathrm{Ar} /{ }^{40} \mathrm{Ar}$ versus ${ }^{39} \mathrm{Ar}{ }^{40} \mathrm{Ar}$ correlation diagram defining a $\left({ }^{40} \mathrm{Ar} /{ }^{36} \mathrm{Ar}\right)_{\mathrm{i}}$ below the atmospheric value of 295.5. Since the data do not plot close to the $\mathrm{x}$-axis, the abcissa intercept is not well constrained. For this reason we favour the weighted mean age of $10.94 \pm 0.23 \mathrm{Ma}$, calculated with the individual ages of three fractions representing $84.57 \%$ of the ${ }^{39} \mathrm{Ar}$ released.

\section{SC 3 mafic dike}

Two laser step-heating experiments were performed on a plagioclase. The sample yielded a pronounced saddle-shaped age spectra, typical of excess argon and was also characterized by low content of radiogenic ${ }^{40} \mathrm{Ar}$. On the basis of the results of the first experiment, a more suitable heating schedule was selected for the second experiment. This allowed us to obtain an age of $10.89 \pm 0.52 \mathrm{Ma}$, using the two fractions (representing $30.61 \%$ of the ${ }^{39} \mathrm{Ar}$ released) that define the base of the saddle. We have confidence in this age, because the combined data of the two experiments, when plotted in the ${ }^{36} \mathrm{Ar} /{ }^{40} \mathrm{Ar}$ vs ${ }^{39} \mathrm{Ar} /{ }^{40} \mathrm{Ar}$ correlation diagram yielded a not so precise, but similar, age of $10.74 \pm 2.59$ Ma. Additionally the $\left({ }^{40} \mathrm{Ar}{ }^{36} \mathrm{Ar}\right)_{\mathrm{i}}=322 \pm 13$, confirms the presence of excess argon in the sample. We therefore prefer the $10.89 \pm 0.52 \mathrm{Ma}$ age as the best age estimate for this mafic dike.

\section{PER 7 basaltic andesite}

A groundmass concentrate was laser step-heated. With the exception of the first fractions of each experiment, similar ${ }^{40} \mathrm{Ar}-{ }^{39} \mathrm{Ar}$ results were obtained for the remaining part of the experiments. The 
discrepancy observed in the age spectra may be attributed to tiny inhomogeneities undetectable under the binocular microscope. Despite that in the two experiments the first fraction was obtained applying 0.5 Watts of laser power, different amounts of ${ }^{39} \mathrm{Ar}$ were released: $~ 14 \%$ and $22.4 \%$. Furthermore the

${ }^{37} \mathrm{Ar}_{\mathrm{Ca}}{ }^{39} \mathrm{Ar}_{\mathrm{K}}$ diagram displays an ascending staircase pattern, indicating that the composition is variable with respect to the calcium and potassium content or that there is more than one mineral phase releasing the argon isotopes. The data cluster in the central part of the ${ }^{36} \mathrm{Ar} /{ }^{40} \mathrm{Ar}$ versus ${ }^{39} \mathrm{Ar} /{ }^{40} \mathrm{Ar}$ correlation diagram. Their distribution does not define a well-constrained isochron age $\left(\mathrm{t}_{\mathrm{c}}=9.76 \pm\right.$ 0.90 Ma with MSWD $=3.57$ for $n=9$ ). For this reason the plateau age of $10.54 \pm 0.20$ Ma calculated with the weighted mean of three consecutive fractions representing $86.73 \%$ of the ${ }^{39} \mathrm{Ar}$ released is our best estimate for this basaltic andesite.

\section{HUA 6 basaltic andesite}

Two laser step-heating experiments were performed on whole rock. For the first experiment, the three fractions released applying up to 1.7 Watts of laser power yielded discordant ages. For the second experiment a modified heating schedule was chosen and an almost flat age espectrum was obtained. The discrepancies between the two experiments in the ages obtained applying $\leq 1.7$ Watts of laser power can be attributed to the different heating schedules used, because when the amount of argon released in them is added, similar ages are obtained: $11.5 \pm 0.8 \mathrm{Ma}$ and $10.8 \pm 1.2 \mathrm{Ma}$, respectively, for the first and second experiment. Therefore, we focus on the argon isotopes released at higer temperature. The weighted mean of the last four fractions of the second experiment yielded an age of $10.45 \pm 0.15 \mathrm{Ma}$ for $75.13 \%$ of the ${ }^{39} \mathrm{Ar}$ released. This age is statistically indistinguishable from the isochron age of $10.61 \pm 0.15 \mathrm{Ma}$ calculated combining the data of the two experiments. Because the straight line defined by the data in the correlation diagram yields a $\left({ }^{40} \mathrm{Ar} /{ }^{36} \mathrm{Ar}\right)_{\mathrm{i}}$ below the atmospheric value of 295.5, we favour the plateau age of $10.45 \pm 0.15 \mathrm{Ma}$, as our best estimate for basaltic andesite HUA 6.

\section{PER 14 basaltic andesite}

More that one gram of groundmass concentrate was step-heated using the temperature controlled Tafurnace and the argon isotopes were measured with the MS-10 mass spectrometer. The argon was released in nine fractions between 500 to $1350^{\circ} \mathrm{C}$. More than $70 \%$ of the argon was released below $800^{\circ} \mathrm{C}$. The age spectrum is somewhat perturbed and saddle-shaped, suggesting the presence of excess argon. The ages obtained for individual fractions does not fulfill the criteria to calculate a plateau age. When plotted in the ${ }^{36} \mathrm{Ar} /{ }^{40} \mathrm{Ar}$ versus ${ }^{39} \mathrm{Ar} /{ }^{40} \mathrm{Ar}$ correlation diagram, the data are scattered about the best straight line $(\mathrm{MSWD}=28)$ calculated with all the fractions. The y-intercept of $\left({ }^{40} \mathrm{Ar} /{ }^{36} \mathrm{Ar}\right)_{\mathrm{i}}=310$ \pm 4 confirms the presence of excess argon. In spite of the data being scattered, we consider the isochron age $10.33 \pm 0.88 \mathrm{Ma}$ to be reliable. Furthermore, on the basis on field observations and geochemical similarity it is posible to correlate basaltic andesite PER 14 with similar samples in the area.

\section{PER 1 hawaiite}

A groundmass concentrate was laser step-heated in two experiments. Because of the low argon content of the sample, only four fractions were collected on each experiment. $\mathrm{The}^{37} \mathrm{Ar}_{\mathrm{C} a}{ }^{39} \mathrm{Ar}_{\mathrm{K}}$ diagram indicates homogeneous composition with respect to calcium to potassium content for $\sim 60 \%$ of the ${ }^{39} \mathrm{Ar}$ released. The last fractions of each experiment yield younger ages with significantly higher values of ${ }^{37} \mathrm{Ar}_{\mathrm{Ca}}{ }^{39} \mathrm{Ar}_{\mathrm{K}}$ (greater than 2), which can be attributed to a different mineral phase. The weighted mean of two fractions with ${ }^{37} \mathrm{Ar}_{\mathrm{C} a}{ }^{39} \mathrm{Ar}_{\mathrm{K}} \sim 0.6$, representing $54.79 \%$ of the ${ }^{39} \mathrm{Ar}$ released, yielded an age of 
$857 \pm 51 \mathrm{Ka}$. The isochron age of $884 \pm 97 \mathrm{Ka}(\mathrm{MSWD}=0.14)$ was obtained with four fractions, the two intermediate fractions of each experiment with ${ }^{37} \mathrm{Ar}_{\mathrm{Ca}}{ }^{39} \mathrm{Ar}_{\mathrm{K}} \sim 0.6$. Our preferred age for sample PER 1 is $884 \pm 97 \mathrm{Ka}$ because it integrates the results of two experiments and uses fractions that have homogeneous composition with respect to ${ }^{37} \mathrm{Ar}_{\mathrm{Ca}}{ }^{39} \mathrm{Ar}_{\mathrm{K}}$.

\section{PER 4 hawaiite}

A groundmass concentrate was laser step-heated in two experiments yielding reproducible results. To improve the precision of the experiment, in the second experiment a larger amount of sample was used. For the last fraction of the second experiment the signal of mass 36 was not measured correctly, therfore no age information can be reported for this fraction. Because the only information missing for this fraction is mass ${ }^{36} \mathrm{Ar}$, it is possible to draw the age spectrum with the available fractions. For both experiments, the bulk of the ${ }^{39} \mathrm{Ar}$ was released applying more than 2 watts of laser power and these were the fractions with the highest ${ }^{40} \mathrm{Ar} *$ content. With the combined data of the two experiments we were able to construct an isochron with age of $853 \pm 80 \mathrm{Ka}(\mathrm{MSWD}=2.56$ for $\mathrm{n}=7)$. The isochron age is statistically indistinguishable from the plateau age of $843 \pm 74 \mathrm{Ka}$ calculated with the weighted mean of the individual ages of three fractions form the second experiment $\left(72.53 \%\right.$ of the ${ }^{39} \mathrm{Ar}$ released). We favour the plateau age of $843 \pm 74 \mathrm{Ka}$ because it is better constrained as it has a MSWD $=0.68$

\section{PER 6 hawaiite}

A groundmass concentrate was laser step-heated in two experiments. Due to the low content of radiogenic ${ }^{40} \mathrm{Ar}$, the indivual ages obtained are not very precise. For the first experiment the bulk of the ${ }^{39} \mathrm{Ar}$ was released in the last two fractions. The weighted mean of their individual ages yielded a $574 \pm$ $113 \mathrm{Ka}$ age. A diferent heating schedule was selected for the second experiment and in this case the

${ }^{39} \mathrm{Ar}$ was distributed evenly in the five fractions collected. The results from the second experiment are more scattered and less precise than the experiment performed in less detail. The data of the two experiments were combined in the ${ }^{36} \mathrm{Ar} /{ }^{40} \mathrm{Ar}$ versus ${ }^{39} \mathrm{Ar} /{ }^{40} \mathrm{Ar}$ correlation diagram. Due to the low radiogenic ${ }^{40} \mathrm{Ar}$ the data cluster close to the y-axis. Two fractions of the second experiment (in the figure, these are identified with letters $g$ and i) were ignored to calculate the isochron age of $585 \pm 82$ $\mathrm{Ka}$. We favour the isochron age because is well defined (MSWD $=1.36$ for $n=8)$ and it incorportates data from the two experiments performed.

\section{CHO 4 basalt}

One gram of groundmass was step-heated with the Ta-furnace and the argon isotopes were measured with the MS-10 mass spectrometer. Only four fractions were collected. The content of radiogenic ${ }^{40} \mathrm{Ar}$ was very low, with a ${ }^{40} \mathrm{Ar} /{ }^{36} \mathrm{Ar}$ close to atmospheric composition. The bulk of the ${ }^{39} \mathrm{Ar}$ was released in the last two fractions. Due to the lack of detail, no plateau can be defined for this sample. The data cluster very close to the $\mathrm{y}$ - axis in the ${ }^{36} \mathrm{Ar} /{ }^{40} \mathrm{Ar}$ versus ${ }^{39} \mathrm{Ar} /{ }^{40} \mathrm{Ar}$ correlation diagram and display an almost horizontal distribution, yielding an unprecise and unreliable isocrhon age of $29 \pm 181 \mathrm{Ka}$. Therefore the best estimate for the sample is taken from the third fraction released at $1000^{\circ} \mathrm{C}$, which contains the bulk of the ${ }^{39}$ Ar released with an age of $138 \pm 107 \mathrm{Ka}$. 


\section{ES 10}

\section{Basltic andesite}

Long $\mathrm{W}$ Lat $\mathrm{N}$

$-105.60023 .862$

Laser step-heating experiments on plagioclase concentrate

\begin{tabular}{|crccccccccc|}
\multicolumn{1}{r|}{ Pwr } & ${ }^{39} \mathrm{Ar} \times 10^{-6}$ & $\mathrm{~F}^{39} \mathrm{Ar}$ & ${ }^{40} \mathrm{Ar}^{*}{ }^{39} \mathrm{Ar}_{\mathrm{K}}$ & $1 \sigma$ & Age in Ma & $1 \sigma$ & & ${ }^{40} \mathrm{Ar}^{*}$ & ${ }^{40} \mathrm{Ar}^{36} \mathrm{Ar}$ & ${ }^{37} \mathrm{Ar}_{\mathrm{Ca}}{ }^{39} \mathrm{Ar}_{\mathrm{K}}$ \\
\hline 1.00 & 37.752 & 0.1072 & 6.11 & 0.68 & 43.87 & 4.80 & $\mathrm{a}$ & 8.11 & 321.59 & 0.871 \\
2.80 & 57.198 & 0.1620 & 4.72 & 0.22 & 33.95 & 1.60 & $\mathrm{~b}$ & 33.57 & 444.86 & 5.114 \\
5.50 & 52.556 & 0.1487 & 4.37 & 0.15 & 31.46 & 1.05 & $\mathrm{c}$ & 60.66 & 751.08 & 6.692 \\
7.50 & 24.994 & 0.0707 & 4.68 & 0.32 & 33.70 & 2.26 & $\mathrm{~d}$ & 66.18 & 873.77 & 6.867 \\
10.00 & 45.306 & 0.1282 & 4.58 & 0.15 & 32.98 & 1.08 & $\mathrm{e}$ & 73.41 & 1111.22 & 6.868 \\
13.00 & 135.418 & 0.3831 & 4.67 & 0.08 & 33.59 & 0.56 & $\mathrm{f}$ & 83.45 & 1785.19 & 7.308 \\
\hline 0.50 & 6.958 & 0.0128 & 7.31 & 3.35 & 52.36 & 23.61 & $\mathrm{~g}$ & 2.34 & 302.57 & 1.486 \\
1.10 & 27.083 & 0.0498 & 5.10 & 0.54 & 36.71 & 3.86 & $\mathrm{~h}$ & 10.05 & 328.53 & 1.070 \\
2.40 & 38.572 & 0.0708 & 4.24 & 0.40 & 30.53 & 2.83 & $\mathrm{i}$ & 15.76 & 350.80 & 4.383 \\
4.50 & 60.510 & 0.1109 & 4.64 & 0.19 & 33.42 & 1.32 & $\mathrm{j}$ & 52.22 & 618.51 & 7.404 \\
8.00 & 85.797 & 0.1573 & 4.64 & 0.11 & 33.42 & 0.79 & $\mathrm{k}$ & 65.57 & 858.29 & 7.271 \\
12.00 & 90.086 & 0.1652 & 4.66 & 0.10 & 33.54 & 0.75 & 1 & 62.08 & 779.17 & 7.017 \\
13.00 & 106.614 & 0.1955 & 4.79 & 0.07 & 34.50 & 0.49 & $\mathrm{~m}$ & 81.44 & 1591.80 & 7.137 \\
14.00 & 129.701 & 0.2377 & 4.70 & 0.07 & 33.83 & 0.49 & $\mathrm{n}$ & 79.83 & 1465.28 & 7.505 \\
\hline
\end{tabular}

Integrated results

\begin{tabular}{|cccccccc|}
${ }^{39} \mathrm{Ar} \times 10^{-6}$ & ${ }^{40} \mathrm{Ar}^{*} /{ }^{39} \mathrm{Ar}_{\mathrm{K}}$ & $1 \sigma$ & Age in $\mathrm{Ma}$ & $1 \sigma$ & ${ }^{40} \mathrm{Ar}^{*}$ & ${ }^{40} \mathrm{Ar} /{ }^{36} \mathrm{Ar}$ & ${ }^{37} \mathrm{Ar}_{\mathrm{Ca}}{ }^{39} \mathrm{Ar}_{\mathrm{K}}$ \\
\hline 35.18 & 4.78 & 0.10 & 34.37 & 0.71 & 32.11 & 435.28 & 6.083 \\
\hline 54.30 & 4.72 & 0.08 & 33.95 & 0.57 & 32.92 & 440.51 & 6.685 \\
\hline
\end{tabular}

$\mathrm{J}=0.004027 \pm 0.000012$

$\mathrm{t}_{\mathrm{p}}=33.92 \pm 0.30 \mathrm{Ma}$

plateau age was calculated with the weighted mean of fractions $\mathrm{j}$ to $\mathrm{n} ; 86.66 \%$ of ${ }^{39} \mathrm{Ar}$ released in 5 consecutive fractions, MSWD $=0.53$

Preferred age $\mathbf{t}_{\mathbf{c}}=\mathbf{3 3 . 5 8} \pm \mathbf{0 . 2 8 ~ M a},\left({ }^{40} \mathrm{Ar} /{ }^{36} \mathrm{Ar}\right)_{\mathrm{i}}=298 \pm 2, \mathrm{MSWD}=1.1$ for $\mathrm{n}=14$ 


\section{HUA 1}

Crystal rich ignimbrite Huajicori-Picachos road

Long W Lat N

$-105.28105 \quad 22.65721$

laser step-heating experiments on biotite concentrate

\begin{tabular}{|c|c|c|c|c|c|c|c|c|c|c|c|}
\hline Pwr & ${ }^{39} \mathrm{Ar} \times 10^{-6}$ & $\mathrm{~F}^{39} \mathrm{Ar}$ & ${ }^{40} \mathrm{Ar}^{*} /{ }^{39} \mathrm{Ar}_{\mathrm{K}}$ & $1 \sigma$ & Age in $\mathrm{Ma}$ & $1 \sigma$ & & & $\%{ }^{40} \mathrm{Ar}^{*}$ & ${ }^{40} \mathrm{Ar} /{ }^{36} \mathrm{Ar}$ & ${ }^{37} \mathrm{Ar}_{\mathrm{Ca}}{ }^{39} \mathrm{Ar}_{\mathrm{K}}$ \\
\hline 0.30 & 56.99 & 0.0069 & 1.81 & 0.42 & 10.73 & 2.48 & $\mathrm{a}$ & $\ddagger$ & 8.33 & 322.35 & 0.075 \\
\hline 0.80 & 304.36 & 0.0370 & 4.10 & 0.14 & 24.20 & 0.82 & $\mathrm{~b}$ & $\dagger$ & 57.36 & 693.04 & 0.033 \\
\hline 1.60 & 280.72 & 0.0341 & 4.33 & 0.11 & 25.54 & 0.66 & $\mathrm{c}$ & $\ddagger$ & 74.86 & 1175.19 & 0.038 \\
\hline 2.80 & 2015.58 & 0.2450 & 4.53 & 0.06 & 26.70 & 0.36 & d & & 87.00 & 2272.21 & 0.046 \\
\hline 5.00 & 5568.78 & 0.6769 & 4.51 & 0.04 & 26.58 & 0.26 & $\mathrm{e}$ & & 91.06 & 3305.52 & 0.025 \\
\hline 0.50 & 121.61 & 0.0181 & 2.52 & 0.27 & 14.93 & 1.57 & $\mathrm{f}$ & $\ddagger$ & 19.05 & 365.05 & 0.121 \\
\hline 1.00 & 106.50 & 0.0158 & 4.19 & 0.13 & 24.69 & 0.77 & g & $\ddagger$ & 55.28 & 660.72 & 0.049 \\
\hline 2.00 & 657.81 & 0.0978 & 4.46 & 0.09 & 26.28 & 0.54 & $\mathrm{~h}$ & $\ddagger$ & 83.58 & 1799.79 & 0.035 \\
\hline 3.00 & 2008.99 & 0.2988 & 4.50 & 0.08 & 26.52 & 0.44 & $\mathrm{i}$ & & 89.54 & 2824.72 & 0.025 \\
\hline 4.00 & 1695.25 & 0.2522 & 4.49 & 0.02 & 26.45 & 0.12 & $\mathrm{j}$ & & 90.10 & 2983.59 & 0.019 \\
\hline 6.00 & 2132.97 & 0.3173 & 4.53 & 0.07 & 26.73 & 0.38 & $\mathrm{k}$ & & 93.29 & 4400.73 & 0.023 \\
\hline
\end{tabular}

Integrated results

\begin{tabular}{|cccccccc|}
${ }^{39} \mathrm{Ar} \times 10^{-6}$ & ${ }^{40} \mathrm{Ar}^{*} /{ }^{39} \mathrm{Ar}_{\mathrm{K}}$ & $1 \sigma$ & Age in $\mathrm{Ma}$ & $1 \sigma$ & $\%{ }^{40} \mathrm{Ar} *$ & ${ }^{40} \mathrm{Ar} /{ }^{36} \mathrm{Ar}$ & ${ }^{37} \mathrm{Ar}_{\mathrm{Ca}}{ }^{39} \mathrm{Ar}_{\mathrm{K}}$ \\
\hline 8226 & 4.47 & 0.03 & 26.38 & 0.24 & 85.38 & 2021.28 & 0.031 \\
\hline 6723 & 4.46 & 0.03 & 26.31 & 0.23 & 86.14 & 2131.31 & 0.026 \\
\hline
\end{tabular}

$\mathrm{J}=0.0032919 \pm 0.0000168$

\section{Preferred age $t_{p}=26.48 \pm 0.15 \mathrm{Ma}$}

the plateau age was calculated with the weighted mean of fractions $\mathrm{h}$ to $\mathrm{k} ; 96.61 \%$ of ${ }^{39} \mathrm{Ar}$ released in 4 consecutive fractions, MSWD $=0.18$

$\mathrm{t}_{\mathrm{c}}=26.51 \pm 0.69 \mathrm{Ma},\left({ }^{40} \mathrm{Ar}{ }^{36} \mathrm{Ar}\right)_{\mathrm{i}}=295 \pm 69, \mathrm{MSWD}=0.32$ for $\mathrm{n}=5$

$\$$ Fractions ignored in the isochron 


\section{TS 16}

Basaltic lava flow, underlying 23.5 ignimbrite east of Las Canoas, Zacatecas

Long W Lat N

$-104.2322 .63$

laser step-heating experiment on plagioclase concentrate

\begin{tabular}{|ccccccccccc|}
\multicolumn{1}{c}{ Pwr } & ${ }^{39} \mathrm{Ar} \times 10^{-6}$ & $\mathrm{~F}^{39} \mathrm{Ar}$ & ${ }^{40} \mathrm{Ar}^{*}{ }^{39} \mathrm{Ar}_{\mathrm{K}}$ & $1 \sigma$ & Age in Ma & $1 \sigma$ & & ${ }^{0}{ }^{40} \mathrm{Ar}^{*}$ & ${ }^{40} \mathrm{Ar}^{36} \mathrm{Ar}$ & ${ }^{37} \operatorname{Ar}_{\mathrm{Ca}}{ }^{39} \mathrm{Ar}_{\mathrm{K}}$ \\
1.30 & 14.483 & 0.0862 & 3.52 & 1.44 & 23.65 & 9.61 & $\mathrm{a}$ & 14.57 & 345.91 & 16.86 \\
2.70 & 26.421 & 0.1571 & 3.32 & 0.35 & 22.32 & 2.33 & $\mathrm{~b}$ & 70.38 & 997.50 & 17.28 \\
4.70 & 19.403 & 0.1154 & 4.27 & 0.60 & 28.71 & 3.99 & $\mathrm{c}$ & 91.96 & 3676.80 & 17.68 \\
5.85 & 8.413 & 0.0500 & 2.99 & 1.27 & 20.12 & 8.52 & $\mathrm{~d}$ & 61.22 & 761.91 & 18.12 \\
7.95 & 9.761 & 0.0580 & 4.30 & 0.95 & 28.88 & 6.30 & $\mathrm{e}$ & 93.38 & 4465.59 & 18.83 \\
12.00 & 89.875 & 0.5334 & 3.63 & 0.12 & 24.42 & 0.82 & $\mathrm{f}$ & 82.04 & 1645.73 & 20.34 \\
\hline
\end{tabular}

Integrated results

\begin{tabular}{|cccccccc|}
${ }^{39} \mathrm{Ar} \times 10^{-6}$ & ${ }^{40} \mathrm{Ar} * /{ }^{39} \mathrm{Ar}_{\mathrm{K}}$ & $1 \sigma$ & Age in Ma & $1 \sigma$ & ${ }^{40}{ }^{40} \mathrm{Ar} *$ & ${ }^{40} \mathrm{Ar} /{ }^{36} \mathrm{Ar}$ & ${ }^{37} \mathrm{Ar}_{\mathrm{Ca}}{ }^{39} \mathrm{Ar}_{\mathrm{K}}$ \\
\hline 166.30 & 3.65 & 0.19 & 24.56 & 1.25 & 58.66 & 714.78 & 19.055 \\
\hline
\end{tabular}

$\mathrm{J}=0.003753 \pm 0.000011$

Preferred age $t_{p}=24.38 \pm 0.75 \mathrm{Ma}$

Weighted mean of fractions a to f; $100 \%$ of ${ }^{39}$ Ar released in 6 consecutive fractions, MSWD $=0.54$

$\mathrm{t}_{\mathrm{c}}=24.43 \pm 0.87 \mathrm{Ma},\left({ }^{40} \mathrm{Ar} /{ }^{36} \mathrm{Ar}\right)_{\mathrm{i}}=293 \pm 21, \mathrm{MSWD}=0.68$ for $\mathrm{n}=6$ 


\section{HUA 2}

\section{Lithic ignimbrite near Picachos}

Long W Lat N

$-105.19123 \quad 22.70874$

laser step-heating experiments on biotite concentrate

\begin{tabular}{|c|c|c|c|c|c|c|c|c|c|c|c|}
\hline Pwr & ${ }^{39} \mathrm{Ar} \times 10^{-6}$ & $\mathrm{~F}^{39} \mathrm{Ar}$ & ${ }^{40} \mathrm{Ar}^{*} /{ }^{39} \mathrm{Ar}_{\mathrm{K}}$ & $1 \sigma$ & Age in Ma & $1 \sigma$ & & & $\%{ }^{40} \mathrm{Ar}^{*}$ & ${ }^{40} \mathrm{Ar} /{ }^{36} \mathrm{Ar}$ & ${ }^{37} \mathrm{Ar}_{\mathrm{Ca}}{ }^{39} \mathrm{Ar}_{\mathrm{K}}$ \\
\hline 0.25 & 196.65 & 0.0224 & 4.66 & 0.50 & 27.48 & 2.93 & $\mathrm{a}$ & $\ddagger$ & 53.22 & 631.65 & 0.095 \\
\hline 0.80 & 515.45 & 0.0588 & 3.70 & 0.11 & 21.86 & 0.67 & $\mathrm{~b}$ & 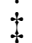 & 60.97 & 757.19 & 0.288 \\
\hline 1.60 & 1359.49 & 0.1551 & 3.88 & 0.05 & 22.89 & 0.27 & $\mathrm{c}$ & & 78.86 & 1397.60 & 0.108 \\
\hline 2.80 & 3722.85 & 0.4246 & 3.92 & 0.04 & 23.12 & 0.22 & d & & 91.41 & 3441.81 & 0.046 \\
\hline 5.00 & 2973.21 & 0.3391 & --- & --- & ---- & ---- & $\mathrm{e}$ & & ---- & ---- & 0.044 \\
\hline 0.50 & 978.41 & 0.0600 & 3.55 & 0.18 & 20.94 & 1.07 & $\mathrm{f}$ & + & 46.63 & 553.73 & 0.128 \\
\hline 1.00 & 1592.75 & 0.0977 & 3.84 & 0.05 & 22.64 & 0.29 & g & & 73.45 & 1113.06 & 0.072 \\
\hline 2.00 & 6321.62 & 0.3879 & 3.90 & 0.04 & 22.99 & 0.22 & $\mathrm{~h}$ & & 87.17 & 2303.44 & 0.053 \\
\hline 2.70 & 3217.38 & 0.1974 & 3.95 & 0.04 & 23.31 & 0.26 & $\mathrm{i}$ & & 89.74 & 2879.45 & 0.063 \\
\hline 4.00 & 3227.12 & 0.1980 & 3.89 & 0.06 & 22.95 & 0.32 & $\mathrm{j}$ & & 93.04 & 4244.08 & 0.054 \\
\hline 6.00 & 960.41 & 0.0589 & 3.89 & 0.06 & 22.97 & 0.38 & $\mathrm{k}$ & & 93.48 & 4534.33 & 0.086 \\
\hline
\end{tabular}

Integrated results

\begin{tabular}{|rccccccc|}
${ }^{39} \mathrm{Ar} \times 10^{-6}$ & ${ }^{40} \mathrm{Ar}^{*}{ }^{39} \mathrm{Ar}_{\mathrm{K}}$ & $1 \sigma$ & $\mathrm{Age}$ in $\mathrm{Ma}$ & $1 \sigma$ & $\%{ }^{40} \mathrm{Ar} *$ & ${ }^{40} \mathrm{Ar} /{ }^{36} \mathrm{Ar}$ & ${ }^{37} \mathrm{Ar}_{\mathrm{Ca}}{ }^{39} \mathrm{Ar}_{\mathrm{K}}$ \\
\hline 8768.0 & ---- & ---- & ---- & --- & ---- & --- & 0.071 \\
\hline 16300.0 & 3.88 & 0.02 & 22.89 & 0.18 & 83.54 & 1794.92 & 0.064 \\
\hline
\end{tabular}

$\mathrm{J}=0.0032919 \pm 0.0000168$

\section{Preferred age $t_{p}=22.99 \pm 0.14$ Ma}

the plateau age was calculated with the weighted mean of fractions $\mathrm{g}$ to $\mathrm{k} ; 94.00 \%$ of ${ }^{39} \mathrm{Ar}$ released in 5 consecutive fractions, MSWD $=0.63$

$\mathrm{t}_{\mathrm{c}}=23.19 \pm 0.27 \mathrm{Ma},\left({ }^{40} \mathrm{Ar} /{ }^{36} \mathrm{Ar}\right)_{\mathrm{i}}=282 \pm 21, \mathrm{MSWD}=0.35$ for $\mathrm{n}=6$

$\$$ Fractions ignored in the isochron

for fraction "e" mass 36 was not tuned properly, no age information can be obtained. 


\section{ESC 7}

Basaltic andesite lava

Escuinapa-Corral de Piedras road, Sinaloa

Long W Lat N

$-105.68 \quad 22.889$

Ta furnace step-heating experiments on plagioclase concentrate

\begin{tabular}{|rcccccccccc|}
\multicolumn{1}{|c}{ Temp ${ }^{\circ} \mathrm{C}$} & ${ }^{39} \mathrm{Arcc} \mathrm{STP} / \mathrm{g}$ & $\mathrm{F}^{39} \mathrm{Ar}$ & ${ }^{40} \mathrm{Ar}^{*}{ }^{39} \mathrm{Ar}_{\mathrm{K}}$ & $1 \sigma$ & $\mathrm{Age}$ in $\mathrm{Ma}$ & $1 \sigma$ & & ${ }^{\circ 0}{ }^{40} \mathrm{Ar}^{*}$ & ${ }^{40} \mathrm{Ar}^{36} \mathrm{Ar}^{37}{ }^{37} \mathrm{Ar}_{\mathrm{Ca}}{ }^{39} \mathrm{Ar}_{\mathrm{K}}$ \\
\hline 600 & $3.20 \mathrm{E}-09$ & 0.0431 & 0.43 & 7.67 & 2.65 & 47.84 & $\mathrm{a}$ & 0.65 & 297.43 & 11.11 \\
800 & $2.35 \mathrm{E}-08$ & 0.3133 & 4.14 & 1.07 & 25.67 & 6.61 & $\mathrm{~b}$ & 19.32 & 366.25 & 43.82 \\
1000 & $2.95 \mathrm{E}-08$ & 0.3932 & 3.26 & 0.80 & 20.24 & 4.97 & $\mathrm{c}$ & 22.65 & 382.02 & 30.79 \\
1100 & $9.60 \mathrm{E}-09$ & 0.1282 & 2.69 & 2.60 & 16.72 & 16.10 & $\mathrm{~d}$ & 6.68 & 316.64 & 28.36 \\
1350 & $9.20 \mathrm{E}-09$ & 0.1221 & 3.80 & 3.02 & 23.61 & 18.62 & $\mathrm{e}$ & 2.93 & 304.41 & 31.80 \\
\hline 550 & $2.30 \mathrm{E}-09$ & 0.0280 & 0.73 & 7.09 & 4.54 & 44.20 & $\mathrm{f}$ & 0.84 & 298.02 & 12.48 \\
700 & $9.70 \mathrm{E}-09$ & 0.1187 & 3.59 & 1.67 & 22.28 & 10.33 & $\mathrm{~g}$ & 17.59 & 358.56 & 34.59 \\
850 & $2.63 \mathrm{E}-08$ & 0.3216 & 3.66 & 0.63 & 22.71 & 3.89 & $\mathrm{~h}$ & 26.33 & 401.11 & 38.52 \\
1000 & $2.32 \mathrm{E}-08$ & 0.2832 & 3.54 & 0.70 & 21.97 & 4.35 & $\mathrm{i}$ & 30.63 & 425.96 & 26.77 \\
1150 & $1.46 \mathrm{E}-08$ & 0.1789 & 3.67 & 1.11 & 22.76 & 6.87 & $\mathrm{j}$ & 22.39 & 380.73 & 22.57 \\
1350 & $5.70 \mathrm{E}-09$ & 0.0696 & 3.32 & 2.95 & 20.62 & 18.23 & $\mathrm{k}$ & 4.30 & 308.77 & 33.05 \\
\hline
\end{tabular}

Integrated results

\begin{tabular}{|cccccccc|}
${ }^{39} \mathrm{ArccSTP} / \mathrm{g}$ & ${ }^{40} \mathrm{Ar}^{*} /{ }^{39} \mathrm{Ar}_{\mathrm{K}}$ & $1 \sigma$ & Age in Ma & $1 \sigma$ & $\%{ }^{40} \mathrm{Ar} *$ & ${ }^{40} \mathrm{Ar} /{ }^{36} \mathrm{Ar}$ & ${ }^{37} \mathrm{Ar}_{\mathrm{Ca}}{ }^{39} \mathrm{Ar}_{\mathrm{K}}$ \\
\hline $7.503 \mathrm{E}-08$ & 3.40 & 0.82 & 21.15 & 5.05 & 9.40 & 326.15 & 33.84 \\
\hline $8.178 \mathrm{E}-08$ & 3.51 & 0.53 & 21.81 & 3.26 & 16.82 & 355.24 & 30.48 \\
\hline
\end{tabular}

$\mathrm{J}=0.0034640 \pm 0.0000196$

$1^{\text {st }}$ exp. sample weight $=0.1987 \mathrm{~g}$

$2^{\text {nd }}$ exp. sample weight $=0.3202 \mathrm{~g}$

Preferred age $t_{p}=22.39 \pm 2.56 \mathrm{Ma}$

the plateau age was calculated with the weighted mean of fractions g to k; $97.20 \%$ of ${ }^{39} \mathrm{Ar}$ released in 5 consecutive fractions, MSWD $<0.01$

$t_{c}=22.35 \pm 2.73 \mathrm{Ma},\left({ }^{40} \mathrm{Ar}{ }^{36}{ }^{A r}\right)_{i}=295 \pm 6, \mathrm{MSWD}=0.10$ for $\mathrm{n}=11$ 


\section{MdCH 10 Dacite}

Nuevo León dome, on top of 21.1 Ma ignimbrite plateau, Nayarit

Long W Lat N

$-105.0622 .652$

laser step-heating experiments on plagioclase sample

\begin{tabular}{|c|c|c|c|c|c|c|c|c|c|c|c|}
\hline Pwr & ${ }^{39} \mathrm{Ar} \times 10^{-6}$ & $\mathrm{~F}^{39} \mathrm{Ar}$ & ${ }^{40} \mathrm{Ar}^{*} /{ }^{39} \mathrm{Ar}_{\mathrm{K}}$ & $1 \sigma$ & Age in $\mathrm{Ma}$ & $1 \sigma$ & & & $\%{ }^{40} \mathrm{Ar}^{*}$ & ${ }^{40} \mathrm{Ar} /{ }^{36} \mathrm{Ar}$ & ${ }^{37} \mathrm{Ar}_{\mathrm{Ca}}{ }^{\beta 9} \mathrm{Ar}_{\mathrm{K}}$ \\
\hline 0.20 & 2.167 & 0.0133 & 12.99 & 9.44 & 79.60 & 56.59 & $\mathrm{a}$ & & 16.81 & 355.19 & 0.466 \\
\hline 0.50 & 7.012 & 0.0431 & ---- & ---- & ---- & ---- & b & $\ddagger$ & ---- & ---- & 2.595 \\
\hline 0.70 & 9.508 & 0.0584 & ---- & ---- & ---- & ---- & $\mathrm{c}$ & $\ddagger$ & ---- & ---- & 3.920 \\
\hline 1.00 & 15.127 & 0.0929 & 3.99 & 0.99 & 24.84 & 6.09 & d & & 93.79 & 4755.49 & 4.985 \\
\hline 2.50 & 37.561 & 0.2307 & 3.32 & 0.27 & 20.69 & 1.70 & $\mathrm{e}$ & & 74.19 & 1144.71 & 4.941 \\
\hline 7.00 & 91.584 & 0.5614 & 3.65 & 0.18 & 22.71 & 1.14 & $\mathrm{f}$ & & 86.37 & 2168.57 & 7.965 \\
\hline 1.00 & 3.395 & 0.0366 & ---- & ---- & ---- & ---- & g & $t$ & ---- & ---- & 3.226 \\
\hline 3.00 & 22.052 & 0.2372 & 3.60 & 0.50 & 22.42 & 3.09 & $\mathrm{~h}$ & & 55.53 & 664.56 & 6.203 \\
\hline 9.00 & 67.594 & 0.7262 & 3.78 & 0.14 & 23.54 & 0.84 & $\mathrm{i}$ & & 87.14 & 2297.16 & 8.362 \\
\hline
\end{tabular}

Integrated results

\begin{tabular}{|rccccccc|}
${ }^{39} \mathrm{Ar} \times 10^{-6}$ & ${ }^{40} \mathrm{Ar}^{*}{ }^{39} \mathrm{Ar}_{\mathrm{K}}$ & $1 \sigma$ & $\mathrm{Age}$ in $\mathrm{Ma}$ & $1 \sigma$ & $\%{ }^{40} \mathrm{Ar}^{*}$ & ${ }^{40} \mathrm{Ar} /{ }^{36} \mathrm{Ar}$ & ${ }^{37} \mathrm{Ar}_{\mathrm{Ca}}{ }^{39} \mathrm{Ar}_{\mathrm{K}}$ \\
\hline 162.3 & ---- & ---- & ---- & --- & ---- & -- & 6.422 \\
\hline 92.58 & ---- & ---- & ---- & ---- & ---- & ---- & 7.662 \\
\hline
\end{tabular}

$\mathrm{J}=0.003473 \pm 0.000013$

$t_{p}=22.15 \pm 0.93 \mathrm{Ma}$; calculated with the weighted mean of fractions $d$ to $f ; 88.50 \%$ of ${ }^{39}$ Ar released in 3 consecutive fractions, MSWD $=0.59$

Preferred age $t_{c}=22.51 \pm 0.90 \mathrm{Ma}$

$\left({ }^{40} \mathrm{Ar} /{ }^{36} \mathrm{Ar}\right)_{\mathrm{i}}=322 \pm 39, \mathrm{MSWD}=0.73$ for $\mathrm{n}=6$

f fraction ignored in the isochron

for fractions “a, b, c, g" mass 36 was not tuned properly, no age information can be obtained and in consequence only ${ }^{39} \mathrm{Ar}$ and ${ }^{37} \mathrm{Ar}$ data can be reported in the integrated results. 


\section{ORO 2}

Ignimbrite, Road to El Cajón Hydro plant

Long W Lat N

$-104.5506721 .45178$

laser step-heating experiments on biotite concentrate

\begin{tabular}{|c|c|c|c|c|c|c|c|c|c|c|c|}
\hline Pwr & ${ }^{39} \mathrm{Ar} \times 10^{-6}$ & $\mathrm{~F}^{39} \mathrm{Ar}$ & ${ }^{40} \mathrm{Ar}^{*} /{ }^{39} \mathrm{Ar}_{\mathrm{K}}$ & $1 \sigma$ & Age in $\mathrm{Ma}$ & $1 \sigma$ & & & $\%{ }^{40} \mathrm{Ar} *$ & ${ }^{40} \mathrm{Ar} /{ }^{36} \mathrm{Ar}$ & ${ }^{37} \mathrm{Ar}_{\mathrm{Ca}}{ }^{39} \mathrm{Ar}_{\mathrm{K}}$ \\
\hline 0.30 & 24.410 & 0.0027 & -1.28 & 2.73 & -7.59 & 16.28 & $\mathrm{a}$ & $\ddagger$ & -6.52 & 277.42 & 0.021 \\
\hline 0.73 & 123.496 & 0.0134 & 3.30 & 0.33 & 19.48 & 1.91 & $\mathrm{~b}$ & $\ddagger$ & 52.31 & 619.56 & 0.289 \\
\hline 1.20 & 500.643 & 0.0544 & 3.43 & 0.06 & 20.26 & 0.37 & $\mathrm{c}$ & \pm & 69.86 & 980.46 & 0.024 \\
\hline 1.80 & 2288.553 & 0.2489 & 3.57 & 0.04 & 21.06 & 0.26 & $\mathrm{~d}$ & & 86.93 & 2261.76 & 0.036 \\
\hline 2.40 & 2928.183 & 0.3185 & 3.56 & 0.04 & 21.02 & 0.22 & $\mathrm{e}$ & & 92.20 & 3790.20 & 0.033 \\
\hline 3.00 & 2105.753 & 0.2290 & 3.53 & 0.04 & 20.85 & 0.24 & $\mathrm{f}$ & & 92.65 & 4020.00 & 0.036 \\
\hline 6.00 & 1223.813 & 0.1331 & 3.53 & 0.04 & 20.87 & 0.24 & g & & 91.53 & 3490.50 & 0.027 \\
\hline
\end{tabular}

Integrated results

\begin{tabular}{|cccccccc|}
${ }^{39} \mathrm{Ar} \times 10^{-6}$ & ${ }^{40} \mathrm{Ar}^{*}{ }^{39} \mathrm{Ar}_{\mathrm{K}}$ & $1 \sigma$ & Age in $\mathrm{Ma}$ & $1 \sigma$ & $\%{ }^{40} \mathrm{Ar}^{*}$ & ${ }^{40} \mathrm{Ar} /{ }^{36} \mathrm{Ar}$ & ${ }^{37} \mathrm{Ar}_{\mathrm{Ca}}{ }^{39} \mathrm{Ar}_{\mathrm{K}}$ \\
\hline 9195.0 & 3.53 & 0.02 & 20.83 & 0.17 & 87.30 & 2326.75 & 0.036 \\
\hline
\end{tabular}

$\mathrm{J}=0.0032919 \pm 0.0000168$

$t_{p}=20.88 \pm 0.12 \mathrm{Ma}$, calculated with the weighted mean of fractions $b$ to $g ; 99.73 \%$ of ${ }^{39}$ Ar released in 6 consecutive fractions, MSWD $=0.85$

Preferred age $\mathbf{t}_{\mathbf{c}}=\mathbf{2 0 . 7 4} \pm \mathbf{0 . 4 4} \mathrm{Ma},\left({ }^{40} \mathrm{Ar} /{ }^{36} \mathrm{Ar}\right)_{\mathrm{i}}=325 \pm 59, \mathrm{MSWD}=0.19$ for $\mathrm{n}=4$

\$ Fractions ignored in the isochron 


\section{MdCH 06}

Rhyolite dome

Laser step-heating experiments on plagioclase concentrate

\begin{tabular}{|rcccccccccc|} 
Pwr & ${ }^{39} \mathrm{Ar} \times 10^{-6}$ & $\mathrm{~F}^{39} \mathrm{Ar}$ & ${ }^{40} \mathrm{Ar}^{*} /{ }^{39} \mathrm{Ar}_{\mathrm{K}}$ & \multicolumn{1}{c}{$1 \sigma$} & Age in Ma & \multicolumn{1}{c}{$1 \sigma$} & & ${ }^{40}{ }^{40} \mathrm{Ar}^{*}$ & ${ }^{40} \mathrm{Ar}^{36} \mathrm{Ar}$ & ${ }^{37} \mathrm{Ar}_{\mathrm{Ca}}{ }^{39} \mathrm{Ar}_{\mathrm{K}}$ \\
0.30 & 0.107 & 0.0009 & 19.38 & 59.05 & 112.68 & 332.74 & $\mathrm{a}$ & 4.43 & 309.21 & 5.48 \\
0.90 & 4.250 & 0.0363 & 15.93 & 5.45 & 93.13 & 31.07 & $\mathrm{~b}$ & 3.22 & 305.34 & 7.14 \\
1.20 & 6.970 & 0.0593 & 2.25 & 1.95 & 13.45 & 11.60 & $\mathrm{c}$ & 3.59 & 306.50 & 14.79 \\
1.70 & 6.838 & 0.0580 & 3.16 & 1.57 & 18.88 & 9.30 & $\mathrm{~d}$ & 7.71 & 320.19 & 18.72 \\
2.30 & 6.870 & 0.0582 & 5.93 & 0.97 & 35.24 & 5.72 & $\mathrm{e}$ & 57.71 & 698.73 & 21.49 \\
4.00 & 9.960 & 0.0852 & 3.74 & 0.73 & 22.30 & 4.32 & $\mathrm{f}$ & 27.87 & 409.68 & 7.18 \\
6.00 & 8.901 & 0.0761 & 3.05 & 0.84 & 18.18 & 4.98 & $\mathrm{~g}$ & 50.57 & 597.83 & 7.25 \\
9.00 & 10.368 & 0.0887 & 2.74 & 0.73 & 16.37 & 4.36 & $\mathrm{~h}$ & 37.84 & 475.36 & 7.02 \\
12.00 & 62.841 & 0.5372 & 3.47 & 0.14 & 20.69 & 0.83 & $\mathrm{i}$ & 49.33 & 583.22 & 7.54 \\
\hline & & & & & & & & & & \\
1.00 & 7.748 & 0.0584 & 14.21 & 3.77 & 83.31 & 21.58 & $\mathrm{j}$ & 4.40 & 309.11 & 16.24 \\
3.00 & 24.290 & 0.1833 & 4.50 & 0.80 & 26.80 & 4.72 & $\mathrm{k}$ & 14.94 & 347.42 & 13.75 \\
6.00 & 10.118 & 0.0766 & 2.95 & 0.74 & 17.58 & 4.41 & 1 & 31.27 & 429.93 & 8.17 \\
7.00 & 7.811 & 0.0592 & 4.33 & 0.64 & 25.81 & 3.77 & $\mathrm{~m}$ & 79.72 & 1457.34 & 7.61 \\
9.50 & 10.937 & 0.0829 & 3.39 & 0.44 & 20.21 & 2.58 & $\mathrm{n}$ & 52.73 & 625.07 & 7.25 \\
10.50 & 22.592 & 0.1711 & 3.60 & 0.33 & 21.45 & 1.96 & $\mathrm{o}$ & 54.89 & 655.11 & 7.97 \\
11.50 & 21.155 & 0.1602 & 3.52 & 0.36 & 21.01 & 2.16 & $\mathrm{p}$ & 42.34 & 512.48 & 7.75 \\
12.50 & 5.023 & 0.0380 & 4.53 & 0.78 & 26.96 & 4.61 & $\mathrm{q}$ & 73.37 & 1109.53 & 8.30 \\
13.00 & 22.487 & 0.1703 & 3.28 & 0.29 & 19.56 & 1.72 & $\mathrm{r}$ & 51.39 & 607.86 & 8.06 \\
\hline
\end{tabular}

Integrated results

\begin{tabular}{|cccccccc|}
${ }^{39} \mathrm{Ar} \times 10^{-6}$ & ${ }^{40} \mathrm{Ar}^{*}{ }^{39} \mathrm{Ar}_{\mathrm{K}}$ & $1 \sigma$ & Age in Ma & $1 \sigma$ & ${ }^{\%}{ }^{40} \mathrm{Ar}^{*}$ & ${ }^{40} \mathrm{Ar} /{ }^{36} \mathrm{Ar}$ & ${ }^{37} \mathrm{Ar}_{\mathrm{Ca}}{ }^{39} \mathrm{Ar}_{\mathrm{K}}$ \\
\hline 116.4 & 3.92 & 0.30 & 23.34 & 1.79 & 12.60 & 338.10 & 9.31 \\
\hline 131.4 & 4.33 & 0.30 & 25.78 & 1.78 & 14.56 & 345.87 & 9.44 \\
\hline
\end{tabular}

$\mathrm{J}=0.003325 \pm 0.000013$

$t_{p}=20.54 \pm 0.79$ Ma, calculated with the weighted mean of fractions $f$ to $i ; 78.72 \%$ of ${ }^{39}$ Ar released in 4 consecutive fractions, MSWD $=0.45$

Preferred age $\mathbf{t}_{\mathbf{c}}=\mathbf{2 0 . 2 5} \pm \mathbf{0 . 6 4} \mathrm{Ma},\left({ }^{40} \mathrm{Ar} /{ }^{36} \mathrm{Ar}\right)_{\mathrm{i}}=304 \pm 2, \mathrm{MSWD}=0.98$ for $\mathrm{n}=18$ 


\section{HUA 5}

\section{Poorly welded ash flow, Huajicori}

Long W Lat N

$-105.3194822 .64773$

laser step-heating experiments on plagioclase concentrate

\begin{tabular}{|rrrrrrrrrrrr|}
\multicolumn{1}{c}{ Pwr } & ${ }^{39} \mathrm{Ar} \times 10^{-6}$ & $\mathrm{~F}^{39} \mathrm{Ar}$ & ${ }^{40} \mathrm{Ar}^{*} /{ }^{39} \mathrm{Ar}_{\mathrm{K}}$ & $1 \sigma$ & Age in Ma & $1 \sigma$ & & ${ }^{40}{ }^{40} \mathrm{Ar}^{*}$ & ${ }^{40} \mathrm{Ar}^{36} \mathrm{Ar}^{37}{ }^{37} \mathrm{Ar}_{\mathrm{Ca}}{ }^{39} \mathrm{Ar}_{\mathrm{K}}$ \\
\hline 1.00 & 16.54 & 0.0069 & 0.40 & 1.69 & 2.37 & 10.00 & $\mathrm{a}$ & $\ddagger$ & 1.02 & 298.53 & 0.345 \\
2.00 & 16.99 & 0.0071 & 2.67 & 0.51 & 15.81 & 3.00 & $\mathrm{~b}$ & $\ddagger$ & 29.99 & 422.07 & 0.619 \\
4.20 & 116.06 & 0.0482 & 3.60 & 0.20 & 21.28 & 1.16 & $\mathrm{c}$ & 82.12 & 1653.06 & 1.674 \\
6.50 & 176.69 & 0.0734 & 3.37 & 0.10 & 19.92 & 0.58 & $\mathrm{~d}$ & 98.71 & 22828.40 & 1.920 \\
8.00 & 2082.41 & 0.8645 & 3.24 & 0.04 & 19.15 & 0.23 & $\mathrm{e}$ & 97.61 & 12350.26 & 2.057 \\
\hline 3.00 & 71.81 & 0.0403 & 2.63 & 0.18 & 15.54 & 1.08 & $\mathrm{f}$ & $\ddagger$ & 17.77 & 359.35 & 0.885 \\
5.00 & 75.00 & 0.0421 & 3.75 & 0.19 & 22.14 & 1.11 & $\mathrm{~g}$ & $\ddagger$ & 48.77 & 2768.21 & 1.688 \\
7.00 & 112.56 & 0.0632 & 3.18 & 0.11 & 18.80 & 0.67 & $\mathrm{~h}$ & 89.26 & 2752.04 & 2.015 \\
8.00 & 1521.12 & 0.8543 & 3.22 & 0.05 & 19.02 & 0.27 & $\mathrm{i}$ & 94.28 & 5165.73 & 2.061 \\
\hline
\end{tabular}

Integrated results

\begin{tabular}{|cccccccc|}
\hline${ }^{39} \mathrm{Ar} \times 10^{-6}$ & ${ }^{40} \mathrm{Ar}^{*}{ }^{39} \mathrm{Ar}_{\mathrm{K}}$ & $1 \sigma$ & Age in Ma & $1 \sigma$ & ${ }^{0}{ }^{40} \mathrm{Ar}^{*}$ & ${ }^{40} \mathrm{Ar} /{ }^{36} \mathrm{Ar}$ & ${ }^{37} \mathrm{Ar}_{\mathrm{Ca}}{ }^{39} \mathrm{Ar}_{\mathrm{K}}$ \\
\hline 2409.0 & 3.25 & 0.04 & 19.17 & 0.24 & 88.51 & 2571.02 & 2.006 \\
\hline 1780.0 & 3.22 & 0.04 & 19.00 & 0.26 & 82.57 & 1695.11 & 1.995 \\
\hline
\end{tabular}

$\mathrm{J}=0.0032919 \pm 0.0000168$

$\mathrm{t}=18.99 \pm 0.27 \mathrm{Ma}$, calculated with the weighted mean of fractions $\mathrm{h}$ and $\mathrm{i} ; 91.75 \%$ of ${ }^{39} \mathrm{Ar}$ released in 2 consecutive fractions, MSWD $=0.09$

Preferred age $\mathbf{t}_{\mathbf{c}}=\mathbf{1 8 . 9 8} \pm \mathbf{0 . 3 0} \mathrm{Ma},\left({ }^{40} \mathrm{Ar} /{ }^{36} \mathrm{Ar}\right)_{\mathrm{i}}=356 \pm 79, \mathrm{MSWD}=1.57$ for $\mathrm{n}=5$

f Fractions ignored in the isochron 


\section{MdCH 09}

Olivine Basalt

El Ryito, Nayarit

Long W Lat N

$-105.17 \quad 22.669$

laser step-heating experiments on plagioclase concentrate

\begin{tabular}{|c|c|c|c|c|c|c|c|c|c|c|c|}
\hline Pwr & ${ }^{39} \mathrm{Ar} \times 10^{-6}$ & $\mathrm{~F}^{39} \mathrm{Ar}$ & ${ }^{40} \mathrm{Ar}^{*}{ }^{39} \mathrm{Ar}_{\mathrm{K}}$ & $1 \sigma$ & Age in $\mathrm{Ma}$ & $1 \sigma$ & & & $\%{ }^{40} \mathrm{Ar}^{*}$ & ${ }^{40} \mathrm{Ar} /{ }^{36} \mathrm{Ar}$ & ${ }^{37} \mathrm{Ar}_{\mathrm{Ca}}{ }^{39} \mathrm{Ar}_{\mathrm{K}}$ \\
\hline 0.50 & 16.481 & 0.0525 & -1.35 & 0.90 & -8.55 & 5.71 & $\mathrm{a}$ & $t$ & -2.07 & 289.51 & 2.241 \\
\hline 1.50 & 32.939 & 0.1043 & 2.63 & 1.19 & 16.52 & 7.43 & $b$ & & 4.55 & 309.59 & 12.793 \\
\hline 3.00 & 38.602 & 0.1218 & 2.68 & 0.32 & 16.85 & 2.02 & $\mathrm{c}$ & & 26.86 & 404.03 & 17.736 \\
\hline 4.50 & 8.219 & 0.0259 & 3.69 & 0.98 & 23.13 & 6.13 & d & & 45.58 & 542.96 & 17.227 \\
\hline 12.00 & 220.482 & 0.6954 & 3.01 & 0.07 & 18.93 & 0.44 & $\mathrm{e}$ & & 65.59 & 858.88 & 18.876 \\
\hline 1.00 & 46.837 & 0.1194 & 4.88 & 0.72 & 30.53 & 4.49 & $\mathrm{f}$ & & 5.42 & 312.45 & 4.704 \\
\hline 3.00 & 63.306 & 0.1600 & 2.18 & 0.33 & 13.70 & 2.06 & $\mathrm{~g}$ & & 18.07 & 360.66 & 17.872 \\
\hline 5.00 & 12.943 & 0.0327 & 3.64 & 0.73 & 22.81 & 4.57 & $\mathrm{~h}$ & & 45.56 & 542.76 & 16.050 \\
\hline 7.00 & 8.890 & 0.0225 & 3.14 & 0.86 & 19.72 & 5.38 & $\mathrm{i}$ & & 41.34 & 503.73 & 14.937 \\
\hline 8.40 & 8.704 & 0.0220 & 2.08 & 0.73 & 13.06 & 4.56 & $\mathrm{j}$ & & 34.64 & 452.11 & 15.960 \\
\hline 10.00 & 40.834 & 0.1032 & 2.82 & 0.18 & 17.71 & 1.15 & $\mathrm{k}$ & & 54.59 & 650.81 & 17.965 \\
\hline 13.00 & 213.901 & 0.5402 & 2.93 & 0.06 & 18.41 & 0.39 & 1 & & 68.02 & 924.02 & 18.551 \\
\hline
\end{tabular}

Integrated results

\begin{tabular}{|cccccccc|}
\hline${ }^{39} \mathrm{Ar} \times 10^{-6}$ & ${ }^{40} \mathrm{Ar}^{*}{ }^{39} \mathrm{Ar}_{\mathrm{K}}$ & $1 \sigma$ & Age in Ma & $1 \sigma$ & ${ }^{0}{ }^{40} \mathrm{Ar} *$ & ${ }^{40} \mathrm{Ar} /{ }^{36} \mathrm{Ar}$ & ${ }^{37} \mathrm{Ar}_{\mathrm{Ca}}{ }^{39} \mathrm{Ar}_{\mathrm{K}}$ \\
\hline 313.2 & 2.72 & 0.15 & 17.10 & 0.97 & 19.33 & 366.32 & 17.186 \\
\hline 391.2 & 3.04 & 0.12 & 19.10 & 0.77 & 18.90 & 364.38 & 16.509 \\
\hline
\end{tabular}

$\mathrm{J}=0.003498 \pm 0.000030$

$\mathrm{t}_{\mathrm{p}}=18.34 \pm 0.39 \mathrm{Ma}$, calculated with the weighted mean of fractions $\mathrm{h}$ to $1 ; 72.06 \%$ of $^{39} \mathrm{Ar}$ released in 5 consecutive fractions, MSWD $=0.67$

Preferred age $\mathbf{t}_{\mathbf{c}}=\mathbf{1 8 . 3 2} \pm \mathbf{0 . 4 0} \mathrm{Ma},\left({ }^{40} \mathrm{Ar} /{ }^{36} \mathrm{Ar}\right)_{\mathrm{i}}=300 \pm 3, \mathrm{MSWD}=1.63$ for $\mathrm{n}=11$

$\$$ fraction ignored in the isochron 


\section{RUIZ 16}

\section{Rhyolitic dome}

Long $\mathrm{W}$ Lat $\mathrm{N}$

$-104.745 \quad 22.155$

Laser step-heating experiments on plagioclase concentrate

\begin{tabular}{|rrrrrrrrrrrr|}
\multicolumn{1}{c}{ Pwr } & ${ }^{39} \mathrm{Ar} \times 10^{-6}$ & $\mathrm{~F}^{39} \mathrm{Ar}$ & ${ }^{40} \mathrm{Ar}^{*}{ }^{39} \mathrm{Ar}_{\mathrm{K}}$ & \multicolumn{1}{c}{$1 \sigma$} & Age in Ma & \multicolumn{1}{c}{$1 \sigma$} & & ${ }^{40} \mathrm{Ar}^{*}$ & ${ }^{40} \mathrm{Ar}^{36} \mathrm{Ar}^{37}{ }^{37} \mathrm{Ar}_{\mathrm{Ca}}{ }^{39} \mathrm{Ar}_{\mathrm{K}}$ \\
0.50 & 0.54 & 0.0009 & -57.13 & 36.53 & -317.69 & 222.12 & $\mathrm{a}$ & + & -10.44 & 267.58 & 0.734 \\
1.20 & 7.68 & 0.0129 & 5.60 & 0.68 & 28.35 & 3.41 & $\mathrm{~b}$ & 30.88 & 427.54 & 0.960 \\
2.40 & 29.89 & 0.0501 & 3.19 & 0.20 & 16.17 & 1.00 & $\mathrm{c}$ & 62.44 & 786.79 & 1.159 \\
3.00 & 22.42 & 0.0376 & 3.45 & 0.25 & 17.51 & 1.25 & $\mathrm{~d}$ & 89.19 & 2734.58 & 1.467 \\
4.00 & 48.58 & 0.0814 & 3.59 & 0.15 & 18.23 & 0.76 & $\mathrm{e}$ & 95.41 & 6436.68 & 1.562 \\
5.00 & 37.37 & 0.0626 & 3.86 & 0.12 & 19.56 & 0.62 & $\mathrm{f}$ & 98.18 & 16260.36 & 1.670 \\
7.00 & 43.73 & 0.0733 & 3.68 & 0.14 & 18.69 & 0.73 & $\mathrm{~g}$ & 90.74 & 3192.09 & 1.691 \\
8.00 & 406.33 & 0.6811 & 3.60 & 0.03 & 18.25 & 0.16 & $\mathrm{~h}$ & 84.67 & 1927.56 & 1.875 \\
\hline 2.00 & 42.96 & 0.0796 & 3.39 & 0.33 & 17.22 & 1.69 & $\mathrm{i}$ & 21.09 & 374.46 & 1.220 \\
5.00 & 103.84 & 0.1923 & 3.53 & 0.07 & 17.90 & 0.36 & $\mathrm{j}$ & 79.15 & 1417.20 & 1.573 \\
6.80 & 54.20 & 0.1003 & 3.63 & 0.12 & 18.42 & 0.60 & $\mathrm{k}$ & 87.76 & 2414.92 & 1.804 \\
7.50 & 30.28 & 0.0561 & 3.60 & 0.22 & 18.27 & 1.10 & 1 & 89.59 & 2838.27 & 1.895 \\
8.50 & 13.18 & 0.0244 & 3.07 & 0.42 & 15.56 & 2.12 & $\mathrm{~m}$ & 81.19 & 1570.64 & 1.806 \\
10.50 & 55.49 & 0.1027 & 3.40 & 0.12 & 17.26 & 0.61 & $\mathrm{n}$ & 78.85 & 1397.34 & 1.896 \\
13.00 & 240.14 & 0.4446 & 3.50 & 0.03 & 17.74 & 0.14 & $\mathrm{o}$ & 83.73 & 1815.85 & 1.893 \\
\hline
\end{tabular}

Integrated results

\begin{tabular}{|cccccccc|}
\hline${ }^{39} \mathrm{Ar} \times 10^{-6}$ & ${ }^{40} \mathrm{Ar}^{*}{ }^{39} \mathrm{Ar}_{\mathrm{K}}$ & $1 \sigma$ & Age in Ma & $1 \sigma$ & ${ }^{40}{ }^{40} \mathrm{Ar}^{*}$ & ${ }^{40} \mathrm{Ar} /{ }^{36} \mathrm{Ar}$ & ${ }^{37} \mathrm{Ar}_{\mathrm{Ca}}{ }^{39} \mathrm{Ar}_{\mathrm{K}}$ \\
\hline 595.9 & 3.56 & 0.05 & 18.08 & 0.24 & 73.09 & 1098.08 & 1.759 \\
\hline 539.5 & 3.49 & 0.04 & 17.73 & 0.21 & 67.57 & 911.24 & 1.767 \\
\hline
\end{tabular}

$\mathrm{J}=0.002826 \pm 0.000003$

the plateau age was calculated with the weighted mean of fractions i to o

$t_{p}=17.76 \pm 0.12 \mathrm{Ma}, 100 \%$ of ${ }^{39} \mathrm{Ar}$ released in 7 consecutive fractions, MSWD $=0.5$

$\mathbf{t}_{\mathbf{c}}=\mathbf{1 7 . 9 1} \pm \mathbf{0 . 2 0} \mathrm{Ma},\left({ }^{40} \mathrm{Ar}{ }^{36} \mathrm{Ar}\right)_{\mathrm{i}}=301 \pm 11, \mathrm{MSWD}=2.4$ for $\mathrm{n}=14$

$\$$ fraction ignored in the isochron given in the figure 


\section{RUIZ 34B}

\section{Rhyolitic dome}

\section{Long $\mathrm{W}$ Lat $\mathrm{N}$}

$-104.822 .059$

Laser step-heating experiments on biotite concentrate

\begin{tabular}{|cccccccccccc|} 
Pwr & ${ }^{39} \mathrm{Ar} \times 10^{-6}$ & $\mathrm{~F}^{39} \mathrm{Ar}$ & ${ }^{40} \mathrm{Ar}^{*}{ }^{39} \mathrm{Ar}_{\mathrm{K}}$ & $1 \sigma$ & Age in Ma & $1 \sigma$ & & ${ }^{40}{ }^{40} \mathrm{Ar}^{*}$ & ${ }^{40} \mathrm{Ar}^{36} \mathrm{Ar}{ }^{37} \mathrm{Ar}_{\mathrm{Ca}}{ }^{39} \mathrm{Ar}_{\mathrm{K}}$ \\
0.30 & 11.533 & 0.0080 & 1.81 & 1.02 & 9.22 & 5.16 & $\mathrm{a}$ & $\ddagger$ & 6.45 & 315.86 & 0.029 \\
0.60 & 32.261 & 0.0225 & 3.48 & 0.28 & 17.67 & 1.42 & $\mathrm{~b}$ & $\ddagger$ & 33.05 & 441.40 & 0.019 \\
0.90 & 32.964 & 0.0230 & 3.08 & 0.23 & 15.62 & 1.14 & $\mathrm{c}$ & $\ddagger$ & 60.85 & 754.87 & 0.012 \\
1.40 & 56.261 & 0.0392 & 3.80 & 0.13 & 19.26 & 0.67 & $\mathrm{~d}$ & 85.25 & 2003.91 & 0.006 \\
1.80 & 57.326 & 0.0399 & 3.46 & 0.10 & 17.55 & 0.51 & $\mathrm{e}$ & 83.73 & 1816.22 & 0.005 \\
2.40 & 124.181 & 0.0865 & 3.62 & 0.06 & 18.35 & 0.31 & $\mathrm{f}$ & 89.85 & 2911.61 & 0.003 \\
3.00 & 133.674 & 0.0931 & 3.51 & 0.05 & 17.81 & 0.26 & $\mathrm{~g}$ & 91.14 & 3334.62 & 0.002 \\
3.80 & 472.930 & 0.3295 & 3.51 & 0.02 & 17.79 & 0.10 & $\mathrm{~h}$ & 93.31 & 4413.78 & 0.001 \\
4.40 & 311.826 & 0.2173 & 3.52 & 0.02 & 17.84 & 0.12 & $\mathrm{i}$ & 95.85 & 7117.52 & 0.001 \\
5.00 & 131.532 & 0.0917 & 3.48 & 0.04 & 17.64 & 0.22 & $\mathrm{j}$ & 95.15 & 6093.75 & 0.001 \\
6.00 & 14.266 & 0.0099 & 3.34 & 0.41 & 16.93 & 2.06 & $\mathrm{k}$ & 84.06 & 1854.36 & 0.000 \\
9.00 & 56.388 & 0.0393 & 3.57 & 0.13 & 18.11 & 0.66 & 1 & 79.35 & 1430.94 & 0.002 \\
\hline
\end{tabular}

Integrated results

\begin{tabular}{|cccccccc|}
${ }^{39} \mathrm{Ar} \times 10^{-6}$ & ${ }^{40} \mathrm{Ar}^{*}{ }^{39} \mathrm{Ar}_{\mathrm{K}}$ & $1 \sigma$ & Age in Ma & $1 \sigma$ & ${ }^{40}{ }^{40} \mathrm{Ar} *$ & ${ }^{40} \mathrm{Ar} /{ }^{36} \mathrm{Ar}$ & ${ }^{37} \mathrm{Ar}_{\mathrm{Ca}}{ }^{39} \mathrm{Ar}_{\mathrm{K}}$ \\
\hline 1435.0 & 3.50 & 0.02 & 17.77 & 0.10 & 83.14 & 1752.75 & 0.002 \\
\hline
\end{tabular}

$\mathrm{J}=0.002826 \pm 0.000003$

the plateau age was calculated with the weighted mean of fractions $\mathrm{f}$ to 1

$\mathrm{t}_{\mathrm{p}}=17.82 \pm 0.07 \mathrm{Ma}, 86.74 \%$ of ${ }^{39}$ Ar released in 7 consecutive fractions, MSWD $=0.68$

$\mathbf{t}_{\mathbf{c}}=\mathbf{1 7 . 5 7} \pm \mathbf{0 . 1 9} \mathrm{Ma},\left({ }^{40} \mathrm{Ar} /{ }^{36} \mathrm{Ar}\right)_{\mathrm{i}}=357 \pm 40, \mathrm{MSWD}=0.79$ for $\mathrm{n}=9$

$\$$ fraction ignored in the isochron given in the figure 


\section{PER 12}

\section{Basaltic andesite, Batequitos-El Divisadero road}

Ta furnace step-heating experiment on groundmass sample

\begin{tabular}{|cccccccccccc|} 
Temp ${ }^{\circ} \mathrm{C}$ & ${ }^{39} \mathrm{Ar} \mathrm{ccSTP} / \mathrm{g}$ & $\mathrm{F}^{39} \mathrm{Ar}$ & ${ }^{40} \mathrm{Ar}^{* 39} \mathrm{Ar}_{\mathrm{K}}$ & $1 \sigma$ & Age in Ma & $1 \sigma$ & & & ${ }^{40}{ }^{40} \mathrm{Ar}^{*}$ & ${ }^{40} \mathrm{Ar}^{36} \mathrm{Ar}^{37}{ }^{37} \operatorname{Ar}_{\mathrm{Ca}^{\beta 9}} \mathrm{Ar}_{\mathrm{K}}$ \\
\hline 500 & $5.257 \mathrm{E}-09$ & 0.0132 & 2.52 & 2.54 & 16.33 & 16.41 & $\mathrm{a}$ & $\ddagger$ & 2.77 & 303.93 & 0.98 \\
600 & $1.170 \mathrm{E}-08$ & 0.0294 & 2.49 & 0.72 & 16.10 & 4.66 & $\mathrm{~b}$ & $\ddagger$ & 10.27 & 329.32 & 1.58 \\
700 & $3.521 \mathrm{E}-08$ & 0.0884 & 3.23 & 0.19 & 20.92 & 1.25 & $\mathrm{c}$ & 42.57 & 514.50 & 1.65 \\
800 & $9.660 \mathrm{E}-08$ & 0.2426 & 2.97 & 0.08 & 19.21 & 0.49 & $\mathrm{~d}$ & 68.15 & 927.77 & 1.66 \\
850 & $6.572 \mathrm{E}-08$ & 0.1651 & 2.84 & 0.08 & 18.38 & 0.54 & $\mathrm{e}$ & 77.35 & 1304.75 & 1.90 \\
900 & $4.805 \mathrm{E}-08$ & 0.1207 & 2.72 & 0.11 & 17.59 & 0.71 & $\mathrm{f}$ & 72.11 & 1059.70 & 2.16 \\
950 & $3.288 \mathrm{E}-08$ & 0.0826 & 2.83 & 0.15 & 18.29 & 0.98 & $\mathrm{~g}$ & 73.63 & 1120.53 & 2.32 \\
1000 & $2.849 \mathrm{E}-08$ & 0.0716 & 2.68 & 0.18 & 17.39 & 1.13 & $\mathrm{~h}$ & 66.33 & 877.55 & 2.36 \\
1100 & $4.610 \mathrm{E}-08$ & 0.1158 & 2.66 & 0.12 & 17.20 & 0.77 & $\mathrm{i}$ & 62.90 & 796.50 & 2.63 \\
1350 & $2.816 \mathrm{E}-08$ & 0.0707 & 2.50 & 0.20 & 16.21 & 1.29 & $\mathrm{j}$ & $\ddagger$ & 48.01 & 568.37 & 13.10 \\
\hline
\end{tabular}

Integrated results

\begin{tabular}{|cccccccc|}
${ }^{39} \mathrm{ArccSTP} / \mathrm{g}$ & ${ }^{40} \mathrm{Ar}^{*}{ }^{39} \mathrm{Ar}_{\mathrm{K}}$ & $1 \sigma$ & Age in Ma & $1 \sigma$ & $\%{ }^{40} \mathrm{Ar}^{*}$ & ${ }^{40} \mathrm{Ar} /{ }^{36} \mathrm{Ar}$ & ${ }^{37} \mathrm{Ar}_{\mathrm{Ca}}{ }^{39} \mathrm{Ar}_{\mathrm{K}}$ \\
\hline $3.982 \mathrm{E}-07$ & 2.82 & 0.12 & 18.25 & 0.85 & 45.73 & 544.48 & 2.78 \\
\hline
\end{tabular}

$\mathrm{J}=0.003608 \pm 0.000078$

sample weight $=1.035 \mathrm{~g}$; estimated $0.58 \% \mathrm{~K}$

$t_{p}=17.78 \pm 0.37 \mathrm{Ma}$; plateau age calculated with the weighted mean of fractions e to $\mathrm{j} ; 62.65 \%$ of

${ }^{39} \mathrm{Ar}$ released in 6 consecutive fractions, $\mathrm{MSWD}=0.75$

Preferred age $\mathbf{t}_{\mathbf{c}}=\mathbf{1 7 . 4 2} \pm \mathbf{0 . 7 7} \mathbf{M a},\left({ }^{40} \mathrm{Ar} /{ }^{36} \mathrm{Ar}\right)_{\mathrm{i}}=335 \pm 19, \mathrm{MSWD}=1.8$ for $\mathrm{n}=7$

$\$$ fractions ignored in the isochron given in the figure 


\section{MDCH 05}

Rhyolite,Cucharas dome

Long W Lat N

$-105.26122 .777$

laser step-heating experiments on groundmass sample

\begin{tabular}{|c|c|c|c|c|c|c|c|c|c|c|c|}
\hline Pwr & ${ }^{39} \mathrm{Ar} \times 10^{-6}$ & $\mathrm{~F}^{39} \mathrm{Ar}$ & ${ }^{40} \mathrm{Ar}^{*} /{ }^{39} \operatorname{Ar}_{\mathrm{K}}$ & $1 \sigma$ & Age in $\mathrm{Ma}$ & $1 \sigma$ & & & $\%{ }^{40} \mathrm{Ar}^{*}$ & ${ }^{40} \mathrm{Ar} /{ }^{36} \mathrm{Ar}$ & ${ }^{37} \mathrm{Ar}_{\mathrm{Ca}}{ }^{39} \mathrm{Ar}_{\mathrm{K}}$ \\
\hline 0.40 & 64.619 & 0.0057 & 11.60 & 1.27 & 75.08 & 8.05 & $\mathrm{a}$ & $t$ & 6.70 & 316.70 & 0.003 \\
\hline 0.84 & 493.293 & 0.0438 & 5.09 & 0.19 & 33.35 & 1.25 & $\mathrm{~b}$ & $\ddagger$ & 16.12 & 352.30 & 0.009 \\
\hline 1.00 & 819.131 & 0.0727 & 3.15 & 0.07 & 20.68 & 0.43 & $\mathrm{c}$ & $\ddagger$ & 36.41 & 464.70 & 0.003 \\
\hline 1.40 & 1308.110 & 0.1160 & 2.98 & 0.03 & 19.58 & 0.22 & d & $\ddagger$ & 48.03 & 568.55 & 0.002 \\
\hline 1.80 & 1774.455 & 0.1574 & 2.80 & 0.02 & 18.42 & 0.12 & e & + & 66.43 & 880.14 & 0.002 \\
\hline 2.22 & 1084.352 & 0.0962 & 2.69 & 0.02 & 17.65 & 0.10 & $\mathrm{f}$ & & 79.48 & 1440.36 & 0.002 \\
\hline 2.40 & 558.693 & 0.0496 & 2.63 & 0.03 & 17.29 & 0.17 & $\mathrm{~g}$ & & 79.16 & 1418.28 & 0.002 \\
\hline 2.90 & 565.139 & 0.0501 & 2.61 & 0.03 & 17.13 & 0.18 & $\mathrm{~h}$ & & 75.53 & 1207.39 & 0.002 \\
\hline 3.50 & 489.728 & 0.0434 & 2.60 & 0.03 & 17.13 & 0.23 & $\mathrm{i}$ & & 71.85 & 1049.80 & 0.002 \\
\hline 4.20 & 525.565 & 0.0466 & 2.61 & 0.03 & 17.19 & 0.19 & $\mathrm{j}$ & & 72.32 & 1067.38 & 0.001 \\
\hline 5.20 & 477.472 & 0.0423 & 2.58 & 0.03 & 16.93 & 0.21 & $\mathrm{k}$ & & 67.27 & 902.98 & 0.001 \\
\hline 6.20 & 509.033 & 0.0451 & 2.67 & 0.03 & 17.57 & 0.20 & 1 & & 66.00 & 869.23 & 0.001 \\
\hline 7.22 & 548.891 & 0.0487 & 2.68 & 0.03 & 17.64 & 0.22 & $\mathrm{~m}$ & & 60.87 & 755.17 & 0.001 \\
\hline 8.20 & 512.379 & 0.0454 & 2.65 & 0.03 & 17.41 & 0.19 & $\mathrm{n}$ & & 62.23 & 782.36 & 0.001 \\
\hline 9.20 & 412.571 & 0.0366 & 2.67 & 0.05 & 17.53 & 0.33 & o & & 62.32 & 784.14 & 0.001 \\
\hline 10.20 & 283.356 & 0.0251 & 2.64 & 0.04 & 17.36 & 0.28 & $\mathrm{p}$ & & 65.12 & 847.30 & 0.001 \\
\hline 12.00 & 243.442 & 0.0216 & 2.60 & 0.05 & 17.08 & 0.32 & $\mathrm{q}$ & & 63.91 & 818.82 & 0.001 \\
\hline 12.50 & 316.356 & 0.0281 & 2.72 & 0.03 & 17.86 & 0.18 & $\mathrm{r}$ & & 74.35 & 1152.26 & 0.001 \\
\hline 13.00 & 288.197 & 0.0256 & 2.59 & 0.05 & 17.01 & 0.36 & $\mathrm{~s}$ & & 58.73 & 715.93 & 0.002 \\
\hline
\end{tabular}

Integrated results

\begin{tabular}{|cccccccc|}
${ }^{39} \mathrm{Ar} \times 10^{-6}$ & ${ }^{40} \mathrm{Ar}^{*}{ }^{39} \mathrm{Ar}_{\mathrm{K}}$ & $1 \sigma$ & Age in Ma & $1 \sigma$ & ${ }^{40}{ }^{40} \mathrm{Ar}^{*}$ & ${ }^{40} \mathrm{Ar} /{ }^{36} \mathrm{Ar}$ & ${ }^{37} \mathrm{Ar}_{\mathrm{Ca}}{ }^{39} \mathrm{Ar}_{\mathrm{K}}$ \\
11270.0 & 2.90 & 0.02 & 19.07 & 0.15 & 43.38 & 521.87 & 0.002 \\
\hline
\end{tabular}

$\mathrm{J}=0.003662 \pm 0.000013$

\section{Preferred age $t_{p}=17.41 \pm 0.07$ Ma}

calculated with the weighted mean of fractions f to s; $60.44 \%$ of ${ }^{39}$ Ar released in 14 consecutive fractions, $\mathrm{MSWD}=1.8$

$\mathrm{t}_{\mathrm{c}}=17.52 \pm 0.24 \mathrm{Ma},\left({ }^{40} \mathrm{Ar} /{ }^{36} \mathrm{Ar}\right)_{\mathrm{i}}=292 \pm 9, \mathrm{MSWD}=2.4$ for $\mathrm{n}=14$

† fraction ignored in the isochron 


\section{SIN 25}

\section{Basalt, El Salto reservoir}

Long $\mathrm{W}$ Lat $\mathrm{N}$

$-106.701 \quad 24.1201$

laser step-heating experiments on whole rock sample

\begin{tabular}{|cccccccccccc|}
$\mathrm{Pwr}$ & ${ }^{39} \mathrm{Ar} \times 10^{-6}$ & $\mathrm{~F}^{39} \mathrm{Ar}$ & ${ }^{40} \mathrm{Ar}^{*}{ }^{39} \mathrm{Ar}_{\mathrm{K}}$ & $1 \sigma$ & Age in Ma & $1 \sigma$ & & ${ }^{40} \mathrm{Ar}^{*}$ & ${ }^{40} \mathrm{Ar}^{36} \mathrm{Ar}^{37}{ }^{37} \mathrm{Ar}_{\mathrm{Ca}}{ }^{39} \mathrm{Ar}_{\mathrm{K}}$ \\
0.20 & 4.403 & 0.0023 & -11.28 & 4.80 & -72.62 & 31.55 & $\mathrm{a}$ & $\ddagger$ & -6.37 & 277.79 & 0.660 \\
0.50 & 29.089 & 0.0150 & 1.58 & 0.49 & 9.97 & 3.08 & $\mathrm{~b}$ & $\ddagger$ & 3.06 & 304.82 & 0.772 \\
0.80 & 88.192 & 0.0456 & 2.17 & 0.22 & 13.62 & 1.40 & $\mathrm{c}$ & $\ddagger$ & 20.25 & 370.53 & 0.825 \\
1.20 & 194.186 & 0.1004 & 2.35 & 0.05 & 14.76 & 0.28 & $\mathrm{~d}$ & 55.85 & 669.30 & 0.815 \\
1.60 & 224.761 & 0.1162 & 2.35 & 0.06 & 14.77 & 0.38 & $\mathrm{e}$ & 67.69 & 914.60 & 0.985 \\
2.00 & 285.318 & 0.1475 & 2.29 & 0.03 & 14.36 & 0.19 & $\mathrm{f}$ & 78.42 & 1369.35 & 1.062 \\
2.50 & 283.876 & 0.1468 & 2.31 & 0.04 & 14.50 & 0.25 & $\mathrm{~g}$ & 85.17 & 1991.96 & 1.087 \\
3.00 & 212.498 & 0.1099 & 2.23 & 0.06 & 14.00 & 0.37 & $\mathrm{~h}$ & 84.09 & 1857.29 & 1.005 \\
3.60 & 198.112 & 0.1025 & 2.20 & 0.05 & 13.84 & 0.30 & $\mathrm{i}$ & 79.26 & 1424.82 & 0.916 \\
5.00 & 220.311 & 0.1139 & 2.25 & 0.04 & 14.14 & 0.25 & $\mathrm{j}$ & 83.13 & 1751.27 & 1.133 \\
6.00 & 76.585 & 0.0396 & 2.24 & 0.11 & 14.10 & 0.69 & $\mathrm{k}$ & 78.86 & 1397.58 & 2.090 \\
8.00 & 116.464 & 0.0602 & 2.17 & 0.10 & 13.65 & 0.65 & 1 & 66.73 & 888.31 & 2.263 \\
\hline
\end{tabular}

Integrated results

\begin{tabular}{|cccccccc|}
${ }^{39} \mathrm{Ar} \times 10^{-6}$ & ${ }^{40} \mathrm{Ar}^{*}{ }^{39} \mathrm{Ar}_{\mathrm{K}}$ & $1 \sigma$ & $\mathrm{Age}$ in $\mathrm{Ma}$ & $1 \sigma$ & $\%{ }^{40} \mathrm{Ar} *$ & ${ }^{40} \mathrm{Ar} /{ }^{36} \mathrm{Ar}$ & ${ }^{37} \mathrm{Ar}_{\mathrm{Ca}}{ }^{39} \mathrm{Ar}_{\mathrm{K}}$ \\
\hline 1932.0 & 2.23 & 0.03 & 14.00 & 0.20 & 49.33 & 583.23 & 1.116 \\
\hline
\end{tabular}

$\mathrm{J}=0.003498 \pm 0.000030$

$\mathrm{t}_{\mathrm{p}}=14.31 \pm 0.11 \mathrm{Ma}$, calculated with the weighted mean of fractions $\mathrm{c}$ to $1 ; 98.27 \%$ of ${ }^{39} \mathrm{Ar}$ released in 10 consecutive fractions, MSWD $=1.07$

Preferred age $\mathbf{t}_{\mathbf{c}}=\mathbf{1 4 . 0 1} \pm \mathbf{0 . 2 3 ~ M a},\left({ }^{40} \mathrm{Ar} /{ }^{36} \mathrm{Ar}\right)_{\mathrm{i}}=315 \pm 11, \mathrm{MSWD}=0.8$ for $\mathrm{n}=9$

$\$$ Fractions ignored in the isochron 


\section{SIN15}

\section{Basaltic lava}

Long $\mathrm{W}$ Lat $\mathrm{N}$

$-107.001 \quad 24.269$

Laser step-heating experiments on groundmass concentrate

\begin{tabular}{|rrrrrrrrrrr|}
\multicolumn{1}{c}{ Pwr } & ${ }^{39} \mathrm{Ar} \times 10^{-6}$ & $\mathrm{~F}^{39} \mathrm{Ar}$ & ${ }^{40} \mathrm{Ar}^{*}{ }^{39} \mathrm{Ar}_{\mathrm{K}}$ & $1 \sigma$ & $\mathrm{Age}$ in $\mathrm{Ma}$ & $1 \sigma$ & & ${ }^{0}{ }^{40} \mathrm{Ar}^{*}$ & ${ }^{40} \mathrm{Ar}^{36} \mathrm{Ar}^{37} \mathrm{Ar}_{\mathrm{Ca}}{ }^{39} \mathrm{Ar}_{\mathrm{K}}$ \\
\hline 0.2 & 7.342 & 0.0088 & 6.37 & 1.95 & 32.18 & 9.77 & $\mathrm{a}$ & 6.56 & 316.24 & 1.11 \\
0.5 & 42.597 & 0.0509 & 3.72 & 0.47 & 18.89 & 2.36 & $\mathrm{~b}$ & 8.60 & 323.31 & 0.46 \\
1.0 & 167.794 & 0.2004 & 2.83 & 0.09 & 14.38 & 0.47 & $\mathrm{c}$ & 41.64 & 506.34 & 0.42 \\
1.3 & 84.817 & 0.1013 & 2.61 & 0.09 & 13.27 & 0.44 & $\mathrm{~d}$ & 62.07 & 778.96 & 0.61 \\
1.6 & 58.648 & 0.0700 & 2.82 & 0.12 & 14.33 & 0.61 & $\mathrm{e}$ & 64.30 & 827.74 & 0.91 \\
2.1 & 50.021 & 0.0597 & 2.84 & 0.13 & 14.42 & 0.64 & $\mathrm{f}$ & 71.49 & 1036.48 & 0.97 \\
3.2 & 98.767 & 0.1179 & 2.76 & 0.09 & 13.99 & 0.44 & $\mathrm{~g}$ & 76.24 & 1243.43 & 0.69 \\
4.8 & 178.338 & 0.2129 & 2.70 & 0.05 & 13.70 & 0.26 & $\mathrm{~h}$ & 75.93 & 1227.69 & 1.06 \\
8.0 & 149.327 & 0.1781 & 2.71 & 0.06 & 13.75 & 0.29 & $\mathrm{i}$ & 69.46 & 967.66 & 2.15 \\
\hline
\end{tabular}

Integrated results

\begin{tabular}{|rccccccc|}
${ }^{39} \mathrm{Ar} \times 10^{-6}$ & ${ }^{40} \mathrm{Ar}^{*}{ }^{39} \mathrm{Ar}_{\mathrm{K}}$ & $1 \sigma$ & Age in Ma & $1 \sigma$ & ${ }^{40}{ }^{40} \mathrm{Ar} *$ & ${ }^{40} \mathrm{Ar} /{ }^{36} \mathrm{Ar}$ & ${ }^{37} \mathrm{Ar}_{\mathrm{Ca}}{ }^{39} \mathrm{Ar}_{\mathrm{K}}$ \\
837.1 & 2.83 & 0.04 & 14.35 & 0.23 & 38.91 & 483.69 & 0.98 \\
\hline
\end{tabular}

$\mathrm{J}=0.002826 \pm 0.000003$

the plateau age was calculated with the weighted mean of fractions $\mathrm{c}$ to $\mathrm{i}$

$t_{p}=13.84 \pm 0.15 \mathrm{Ma}, 94.03 \%$ of ${ }^{39}$ Ar released in 7 consecutive fractions, MSWD $=0.82$

$\mathbf{t}_{\mathbf{c}}=\mathbf{1 3 . 6 2} \pm \mathbf{0 . 1 7} \mathrm{Ma},\left({ }^{40} \mathrm{Ar} /{ }^{36} \mathrm{Ar}\right)_{\mathrm{i}}=304 \pm 3, \mathrm{MSWD}=0.56$ for $\mathrm{n}=9$ 


\section{DANA 46a}

Basalt, Nayarit scarp

Long W Lat N

$-106.63 \quad 22.44$

laser one-step fusion experiments on whole rock, irradiation CIC 41

\begin{tabular}{|c|c|c|c|c|c|c|c|c|c|c|}
\hline Pwr & ${ }^{39} \mathrm{Ar} \times 10^{-6}$ & ${ }^{40} \mathrm{Ar}^{*} /{ }^{39} \mathrm{Ar}_{\mathrm{K}}$ & $1 \sigma$ & Age in $\mathrm{Ma}$ & $1 \sigma$ & & & $\%{ }^{40} \mathrm{Ar}^{*}$ & ${ }^{40} \mathrm{Ar} /{ }^{36} \mathrm{Ar}$ & ${ }^{37} \mathrm{Ar}_{\mathrm{Ca}}{ }^{39} \mathrm{Ar}_{\mathrm{K}}$ \\
\hline 3.00 & 506.308 & 1.06 & 0.17 & 9.26 & 1.44 & $\mathrm{a}$ & $t$ & 22.20 & 379.83 & 8.619 \\
\hline 3.00 & 238.204 & 1.44 & 0.20 & 12.58 & 1.76 & $\mathrm{~b}$ & & 38.98 & 484.24 & 3.569 \\
\hline 3.00 & 284.893 & 1.29 & 0.24 & 11.27 & 2.13 & c & & 20.29 & 370.73 & 4.879 \\
\hline 5.00 & 2289.683 & 1.40 & 0.04 & 12.18 & 0.33 & $\mathrm{~d}$ & & 55.37 & 662.06 & 0.543 \\
\hline 4.00 & 1228.054 & 1.26 & 0.13 & 11.02 & 1.16 & e & & 24.22 & 389.96 & 3.499 \\
\hline 4.00 & 1454.440 & 1.37 & 0.09 & 11.91 & 0.82 & $\mathrm{f}$ & & 29.92 & 421.64 & 2.287 \\
\hline 3.00 & 3417.317 & 1.35 & 0.03 & 11.82 & 0.29 & g & & 39.90 & 491.71 & 1.265 \\
\hline 3.00 & 1613.156 & 1.26 & 0.12 & 10.96 & 1.01 & $\mathrm{~h}$ & & 21.34 & 375.66 & 3.386 \\
\hline
\end{tabular}

$\mathrm{J}=0.004851 \pm 0.000007$

laser one-step fusion experiments on whole rock, irradiation CIC 44

\begin{tabular}{|lcccccccccc|}
\multicolumn{1}{c}{ Pwr } & ${ }^{39} \mathrm{Ar} \times 10^{-6}$ & $\mathrm{~F}^{39} \mathrm{Ar}$ & ${ }^{40} \mathrm{Ar}^{*} /{ }^{39} \mathrm{Ar}_{\mathrm{K}}$ & $1 \sigma$ & Age in Ma & $1 \sigma$ & & ${ }^{40} \mathrm{Ar}^{*}$ & ${ }^{40} \mathrm{Ar}^{36} \mathrm{Ar}$ & ${ }^{37} \operatorname{Ar}_{\mathrm{Ca}}{ }^{39} \mathrm{Ar}_{\mathrm{K}}$ \\
\hline 5.00 & 2023.918 & 0.1819 & 1.41 & 0.07 & 11.16 & 0.57 & $\mathrm{i}$ & 26.95 & 404.50 & 2.274 \\
6.00 & 2638.512 & 0.2372 & 1.39 & 0.05 & 10.99 & 0.42 & $\mathrm{j}$ & 32.72 & 439.21 & 1.690 \\
6.13 & 2212.066 & 0.1989 & 1.43 & 0.07 & 11.28 & 0.53 & $\mathrm{k}$ & 32.26 & 436.26 & 1.359 \\
4.07 & 2181.523 & 0.1960 & 1.45 & 0.07 & 11.43 & 0.55 & 1 & 32.84 & 440.02 & 2.467 \\
6.00 & 2069.463 & 0.1860 & 1.45 & 0.08 & 11.45 & 0.63 & $\mathrm{~m}$ & 26.32 & 401.05 & 2.275 \\
\hline
\end{tabular}

$\mathrm{J}=0.004389 \pm 0.000015$

Weighted mean of fractions a to $\mathrm{m} ; \mathrm{t}=11.59 \pm 0.31 \mathrm{Ma} ; \mathrm{MSWD}=0.89$

laser step-heating experiments on whole rock, irradiation CIC 44

\begin{tabular}{|c|c|c|c|c|c|c|c|c|c|c|c|}
\hline Pwr & ${ }^{39} \mathrm{Ar} \times 10^{-6}$ & $\mathrm{~F}^{39} \mathrm{Ar}$ & ${ }^{40} \mathrm{Ar}^{*} /{ }^{39} \mathrm{Ar}_{\mathrm{K}}$ & $1 \sigma$ & Age in $\mathrm{Ma}$ & $1 \sigma$ & & & $\%{ }^{40} \mathrm{Ar} *$ & ${ }^{40} \mathrm{Ar} /{ }^{36} \mathrm{Ar}$ & ${ }^{37} \mathrm{Ar}_{\mathrm{Ca}}{ }^{39} \mathrm{Ar}_{\mathrm{K}}$ \\
\hline 0.18 & 102.766 & 0.0252 & 0.87 & 1.43 & 6.91 & 11.25 & $\mathrm{n}$ & & 1.29 & 299.36 & 2.698 \\
\hline 0.41 & 263.267 & 0.0646 & 1.55 & 0.32 & 12.20 & 2.52 & o & & 12.43 & 337.43 & 1.352 \\
\hline 0.77 & 432.804 & 0.1061 & 0.97 & 0.13 & 7.68 & 1.05 & $\mathrm{p}$ & $\ddagger$ & 16.75 & 354.94 & 1.333 \\
\hline 1.10 & 555.221 & 0.1362 & 1.48 & 0.10 & 11.68 & 0.81 & $\mathrm{q}$ & & 48.92 & 578.49 & 1.032 \\
\hline 6.00 & 2723.589 & 0.6679 & 1.44 & 0.05 & 11.34 & 0.36 & $\mathrm{r}$ & & 46.87 & 556.14 & 1.483 \\
\hline
\end{tabular}

Integrated results

\begin{tabular}{|cccccccc|}
${ }^{39} \mathrm{Ar} \times 10^{-6}$ & ${ }^{40} \mathrm{Ar}^{*} /{ }^{39} \mathrm{Ar}_{\mathrm{K}}$ & $1 \sigma$ & Age in Ma & $1 \sigma$ & $\%{ }^{40} \mathrm{Ar} *$ & ${ }^{40} \mathrm{Ar} /{ }^{36} \mathrm{Ar}$ & ${ }^{37} \mathrm{Ar}_{\mathrm{Ca}}{ }^{39} \mathrm{Ar}_{\mathrm{K}}$ \\
4074.0 & 1.39 & 0.06 & 10.94 & 0.45 & 24.83 & 393.09 & 1.428 \\
\hline
\end{tabular}

$\mathrm{J}=0.004389 \pm 0.000015$ 


\section{DANA 46a}

Basalt, Nayarit scarp

Long W Lat $\mathrm{N}$

$-106.6322 .44$

Ta furnace step-heating experiment on whole rock sample, irradiation CIC 41

\begin{tabular}{|c|c|c|c|c|c|c|c|c|c|c|}
\hline Temp ${ }^{\circ} \mathrm{C}$ & ${ }^{39} \mathrm{Ar} \mathrm{cc} \mathrm{STP} / \mathrm{g}$ & $\mathrm{F}^{39} \mathrm{Ar}$ & ${ }^{40} \mathrm{Ar}^{*}{ }^{\beta 9} \mathrm{Ar}_{\mathrm{K}}$ & $1 \sigma$ & Age in $\mathrm{Ma}$ & $1 \sigma$ & & $\%{ }^{40} \mathrm{Ar}^{*}$ & ${ }^{40} \mathrm{Ar} /{ }^{36} \mathrm{Ar}$ & ${ }^{37} \mathrm{Ar}_{\mathrm{Ca}}{ }^{39} \mathrm{Ar}_{\mathrm{K}}$ \\
\hline 600 & $4.454 \mathrm{E}-08$ & 0.0429 & 1.23 & 0.41 & 10.71 & 3.55 & $\mathrm{~s}$ & 6.83 & 317.17 & 0.97 \\
\hline 700 & $1.085 \mathrm{E}-07$ & 0.1044 & 1.40 & 0.17 & 12.25 & 1.43 & $\mathrm{t}$ & 26.47 & 401.87 & 0.50 \\
\hline 900 & $2.104 \mathrm{E}-07$ & 0.2025 & 1.37 & 0.09 & 11.98 & 0.74 & $\mathrm{u}$ & 39.17 & 485.82 & 1.01 \\
\hline 1000 & $2.448 \mathrm{E}-07$ & 0.2356 & 1.36 & 0.07 & 11.86 & 0.63 & $\mathrm{v}$ & 56.49 & 679.09 & 0.64 \\
\hline 1350 & $4.308 \mathrm{E}-07$ & 0.4146 & 1.39 & 0.04 & 12.10 & 0.38 & $\mathrm{w}$ & 37.50 & 472.80 & 2.74 \\
\hline
\end{tabular}

Integrated results

\begin{tabular}{|cccccccc|}
${ }^{39} \mathrm{Arcc}$ STP $/ \mathrm{g}$ & ${ }^{40} \mathrm{Ar}^{*} /{ }^{39} \mathrm{Ar}_{\mathrm{K}}$ & $1 \sigma$ & Age in Ma & $1 \sigma$ & ${ }^{\%}{ }^{40} \mathrm{Ar}^{*}$ & ${ }^{40} \mathrm{Ar} /{ }^{36} \mathrm{Ar}$ & ${ }^{37} \mathrm{Ar}_{\mathrm{Ca}}{ }^{39} \mathrm{Ar}_{\mathrm{K}}$ \\
\hline $1.040 \mathrm{E}-06$ & 1.37 & 0.04 & 11.98 & 0.36 & 33.20 & 442.38 & 1.59 \\
\hline
\end{tabular}

$\mathrm{J}=0.004851 \pm 0.000007$

sample weight $=0.3107 \mathrm{~g}$; estimated $1.12 \% \mathrm{~K}$

$t_{p}=11.97 \pm 0.30 \mathrm{Ma}$, calculated with the weighted mean of fractions $\mathrm{s}$ to $\mathrm{w} ; 100 \%$ of ${ }^{39} \mathrm{Ar}$ released in 5 consecutive fractions, MSWD $=0.08$

Preferred age $t_{\mathbf{c}}=\mathbf{1 1 . 9 6} \pm \mathbf{0 . 2 6} \mathrm{Ma},\left({ }^{40} \mathrm{Ar}{ }^{\beta 6} \mathrm{Ar}\right)_{\mathrm{i}}=291 \pm 3, \mathrm{MSWD}=0.50$ for $\mathrm{n}=21$

$\$$ Fractions ignored in the isochron 


\section{Roca 3J 5}

Lithic crystal tuff, Tamayo Dome

Long $\mathrm{W}$ Lat $\mathrm{N}$

$-107.34 \quad 23.06$

laser step-heating experiments on feldspar concentrate

\begin{tabular}{|rrrrrrrrrrr|}
\multicolumn{1}{r}{ Pwr } & ${ }^{39} \mathrm{Ar} \times 10^{-6}$ & $\mathrm{~F}^{39} \mathrm{Ar}$ & ${ }^{40} \mathrm{Ar}^{*}{ }^{39} \mathrm{Ar}_{\mathrm{K}}$ & $1 \sigma$ & Age in Ma & $1 \sigma$ & & ${ }^{40}{ }^{40} \mathrm{Ar}^{*}$ & ${ }^{40} \mathrm{Ar}^{36} \mathrm{Ar}^{37}{ }^{37} \mathrm{Ar}_{\mathrm{Ca}}{ }^{39} \mathrm{Ar}_{\mathrm{K}}$ \\
\hline 2.00 & 165.055 & 0.0769 & 1.68 & 0.10 & 11.14 & 0.66 & $\mathrm{a}$ & 46.75 & 554.93 & 0.192 \\
3.70 & 289.830 & 0.1350 & 1.69 & 0.04 & 11.22 & 0.26 & $\mathrm{~b}$ & 75.28 & 1195.18 & 0.159 \\
5.20 & 271.057 & 0.1262 & 1.73 & 0.05 & 11.49 & 0.35 & $\mathrm{c}$ & 87.31 & 2328.46 & 0.142 \\
6.70 & 247.692 & 0.1154 & 1.83 & 0.05 & 12.15 & 0.34 & $\mathrm{~d}$ & 97.72 & 12955.00 & 0.126 \\
12.00 & 1173.434 & 0.5465 & 1.74 & 0.01 & 11.54 & 0.09 & $\mathrm{e}$ & 95.77 & 6993.56 & 0.127 \\
\hline 3.00 & 415.658 & 0.1507 & 1.71 & 0.03 & 11.36 & 0.23 & $\mathrm{f}$ & 57.08 & 688.57 & 0.213 \\
4.50 & 398.701 & 0.1446 & 1.75 & 0.03 & 11.60 & 0.21 & $\mathrm{~g}$ & 82.56 & 1694.40 & 0.154 \\
6.00 & 500.087 & 0.1813 & 1.78 & 0.02 & 11.79 & 0.11 & $\mathrm{~h}$ & 89.32 & 2766.32 & 0.144 \\
7.50 & 293.994 & 0.1066 & 1.83 & 0.03 & 12.12 & 0.22 & $\mathrm{i}$ & 99.13 & 33993.77 & 0.145 \\
9.80 & 292.731 & 0.1061 & 1.78 & 0.03 & 11.79 & 0.18 & $\mathrm{j}$ & 99.05 & 31065.67 & 0.136 \\
13.00 & 856.988 & 0.3107 & 1.75 & 0.01 & 11.63 & 0.08 & $\mathrm{k}$ & 96.18 & 7728.28 & 0.132 \\
\hline
\end{tabular}

Integrated results

\begin{tabular}{|cccccccc|}
${ }^{39} \mathrm{Ar} \times 10^{-6}$ & ${ }^{40} \mathrm{Ar}^{*} /{ }^{39} \mathrm{Ar}_{\mathrm{K}}$ & $1 \sigma$ & Age in Ma & $1 \sigma$ & ${ }^{0}{ }^{40} \mathrm{Ar} *$ & ${ }^{40} \mathrm{Ar} /{ }^{36} \mathrm{Ar}$ & ${ }^{37} \mathrm{Ar}_{\mathrm{Ca}}{ }^{39} \mathrm{Ar}_{\mathrm{K}}$ \\
\hline 2147.0 & 1.74 & 0.02 & 11.53 & 0.12 & 85.25 & 2003.76 & 0.138 \\
\hline 2758.0 & 1.76 & 0.01 & 11.68 & 0.09 & 84.99 & 1968.15 & 0.152 \\
\hline
\end{tabular}

$\mathrm{J}=0.003689 \pm 0.000018$

\section{Preferred age $t_{p}=11.70 \pm 0.07 \mathrm{Ma}$,}

calculated with the weighted mean of fractions $\mathrm{f}$ to $\mathrm{k} ; 100 \%$ of ${ }^{39} \mathrm{Ar}$ released in 6 consecutive fractions, MSWD = 1.5

$\mathrm{t}_{\mathrm{c}}=11.69 \pm 0.08 \mathrm{Ma},\left({ }^{40} \mathrm{Ar} /{ }^{36} \mathrm{Ar}\right)_{\mathrm{i}}=284 \pm 8, \mathrm{MSWD}=1.5$ for $\mathrm{n}=11$ 


\section{LM 2 \\ Dacite, Sierra Navachiste, El Muellecito}

$\begin{array}{ll}\text { Long W } & \text { Lat N } \\ -108.98 & 25.58\end{array}$

laser step-heating experiments on groundmass sample

\begin{tabular}{|cccccccccccc|} 
Pwr & ${ }^{39} \mathrm{Ar} \times 10^{-6}$ & $\mathrm{~F}^{39} \mathrm{Ar}$ & ${ }^{40} \mathrm{Ar}^{*} /{ }^{39} \mathrm{Ar}_{\mathrm{K}}$ & $1 \sigma$ & $\mathrm{Age}$ in Ma & $1 \sigma$ & & ${ }^{40}{ }^{40} \mathrm{Ar}^{*}$ & ${ }^{40} \mathrm{Ar} /{ }^{36} \mathrm{Ar}$ & ${ }^{37} \mathrm{Ar}_{\mathrm{Ca}}{ }^{39} \mathrm{Ar}_{\mathrm{K}}$ \\
0.50 & 71.743 & 0.0365 & 66.04 & 3.60 & 298.84 & 15.00 & $\mathrm{a}$ & $\ddagger$ & 34.52 & 451.27 & 1.201 \\
0.90 & 208.660 & 0.1061 & 9.45 & 0.65 & 45.92 & 3.10 & $\mathrm{~b}$ & 31.49 & 431.32 & 0.644 \\
1.70 & 534.693 & 0.2719 & 3.35 & 0.22 & 16.41 & 1.08 & $\mathrm{c}$ & 51.75 & 612.40 & 0.455 \\
3.50 & 764.359 & 0.3887 & 3.03 & 0.19 & 14.85 & 0.94 & $\mathrm{~d}$ & 57.79 & 700.06 & 0.503 \\
6.00 & 387.392 & 0.1969 & 5.39 & 0.70 & 26.33 & 3.41 & $\mathrm{e}$ & $\ddagger$ & 51.88 & 614.06 & 1.046 \\
\hline 0.30 & 87.116 & 0.0297 & 58.41 & 8.66 & 266.73 & 36.77 & $\mathrm{f}$ & $\ddagger$ & 24.50 & 391.40 & 3.793 \\
0.60 & 217.370 & 0.0743 & 17.84 & 2.16 & 85.73 & 10.14 & $\mathrm{~g}$ & 32.30 & 436.48 & 0.695 \\
1.20 & 519.138 & 0.1775 & 3.97 & 0.56 & 19.43 & 2.70 & $\mathrm{~h}$ & 49.25 & 582.31 & 0.291 \\
2.00 & 976.263 & 0.3338 & 2.89 & 0.16 & 14.16 & 0.80 & $\mathrm{i}$ & 75.91 & 1226.58 & 0.311 \\
3.00 & 532.749 & 0.1821 & 2.72 & 0.21 & 13.34 & 1.01 & $\mathrm{j}$ & 53.68 & 638.00 & 0.448 \\
4.00 & 196.403 & 0.0671 & 4.12 & 0.51 & 20.16 & 2.50 & $\mathrm{k}$ & 64.97 & 843.66 & 0.460 \\
5.00 & 194.278 & 0.0664 & 5.51 & 0.69 & 26.89 & 3.35 & 1 & $\ddagger$ & 73.79 & 1127.49 & 0.730 \\
7.00 & 201.825 & 0.0690 & 6.39 & 0.81 & 31.20 & 3.94 & $\mathrm{~m}$ & $\ddagger$ & 65.51 & 856.69 & 1.401 \\
\hline
\end{tabular}

Integrated results

\begin{tabular}{|cccccccc|}
${ }^{39} \mathrm{Ar} \times 10^{-6}$ & ${ }^{40} \mathrm{Ar}^{*} /{ }^{39} \mathrm{Ar}_{\mathrm{K}}$ & $1 \sigma$ & Age in Ma & $1 \sigma$ & ${ }^{0}{ }^{40} \mathrm{Ar}^{*}$ & ${ }^{40} \mathrm{Ar} /{ }^{36} \mathrm{Ar}$ & ${ }^{37} \mathrm{Ar}_{\mathrm{Ca}}{ }^{39} \mathrm{Ar}_{\mathrm{K}}$ \\
\hline 1966.0 & 6.56 & 0.31 & 32.00 & 1.55 & 40.99 & 500.78 & 0.637 \\
\hline 2924.0 & 6.31 & 0.40 & 30.79 & 1.96 & 38.45 & 480.12 & 0.577 \\
\hline
\end{tabular}

$\mathrm{J}=0.002728 \pm 0.000040$

$\mathrm{t}=13.84 \pm 0.64 \mathrm{Ma}$,

calculated with the weighted mean of fractions $i$ and $j ; 51.59 \%$ of ${ }^{39}$ Ar released in 2 consecutive fractions, MSWD $=0.4$

Preferred age $\left.\mathbf{t}_{\mathbf{c}}=\mathbf{1 1 . 3 4} \pm \mathbf{0 . 7 9} \mathrm{Ma},\left({ }^{40} \mathrm{Ar} /{ }^{36} \mathrm{Ar}\right)\right)_{\mathrm{i}}=400 \pm 10, \mathrm{MSWD}=1.22$ for $\mathrm{n}=8$

+ Fractions ignored in the isochron 


\section{Roca 24J 33}

Microdiorite, S of Pescadero transform

Long W Lat $\mathrm{N}$

$-109.0424 .02$

laser step-heating experiments on hornblende concentrate

\begin{tabular}{|rrrcccccccc|}
\multicolumn{1}{c}{ Pwr } & ${ }^{39} \mathrm{Ar} \times 10^{-6}$ & $\mathrm{~F}^{39} \mathrm{Ar}$ & ${ }^{40} \mathrm{Ar}^{*}{ }^{39} \mathrm{Ar}_{\mathrm{K}}$ & $1 \sigma$ & Age in Ma & $1 \sigma$ & & ${ }^{40} \mathrm{Ar}^{*}$ & ${ }^{40} \mathrm{Ar}^{36} \mathrm{Ar}$ & ${ }^{37} \mathrm{Ar}_{\mathrm{Ca}}{ }^{39} \mathrm{Ar}_{\mathrm{K}}$ \\
\hline 0.20 & 3.467 & 0.0033 & 31.33 & 7.76 & 185.17 & 43.60 & $\mathrm{a}$ & 7.16 & 318.30 & 2.118 \\
0.80 & 83.358 & 0.0791 & 4.83 & 0.95 & 29.80 & 5.79 & $\mathrm{~b}$ & 8.78 & 323.94 & 1.635 \\
1.40 & 267.240 & 0.2536 & 2.36 & 0.15 & 14.63 & 0.95 & $\mathrm{c}$ & 19.68 & 367.90 & 1.952 \\
2.00 & 278.722 & 0.2643 & 1.88 & 0.06 & 11.65 & 0.37 & $\mathrm{~d}$ & 47.13 & 558.91 & 2.757 \\
2.80 & 232.719 & 0.2208 & 2.05 & 0.08 & 12.74 & 0.47 & $\mathrm{e}$ & 59.69 & 733.03 & 2.030 \\
4.00 & 94.286 & 0.0894 & 1.86 & 0.18 & 11.52 & 1.14 & $\mathrm{f}$ & 40.90 & 499.96 & 3.114 \\
6.00 & 94.584 & 0.0895 & 2.11 & 0.15 & 13.08 & 0.91 & $\mathrm{~g}$ & 28.83 & 415.19 & 6.289 \\
\hline
\end{tabular}

Integrated results

\begin{tabular}{|cccccccc|}
${ }^{39} \mathrm{Ar} \times 10^{-6}$ & ${ }^{40} \mathrm{Ar}^{*} /{ }^{39} \mathrm{Ar}_{\mathrm{K}}$ & $1 \sigma$ & Age in Ma & $1 \sigma$ & ${ }^{0}{ }^{40} \mathrm{Ar}^{*}$ & ${ }^{40} \mathrm{Ar} /{ }^{36} \mathrm{Ar}$ & ${ }^{37} \mathrm{Ar}_{\mathrm{Ca}}{ }^{39} \mathrm{Ar}_{\mathrm{K}}$ \\
\hline 1053.0 & 2.39 & 0.10 & 14.80 & 0.62 & 20.41 & 371.26 & 2.649 \\
\hline
\end{tabular}

$\mathrm{J}=0.00345 \pm 0.000025$

Preferred age $t_{c}=11.29 \pm 0.37 \mathrm{Ma}$

$\left({ }^{40} \mathrm{Ar} /{ }^{36} \mathrm{Ar}\right)_{\mathrm{i}}=313 \pm 4, \mathrm{MSWD}=1.12$ for $\mathrm{n}=7$

$\mathrm{W}_{\mathrm{m}}=12.14 \pm 0.34 \mathrm{Ma}$, calculated with the weighted mean of fractions $\mathrm{d}$ to $\mathrm{g} ; 66.40 \%$ of ${ }^{39} \mathrm{Ar}$ released in 4 consecutive fractions, MSWD $=1.53$ 


\section{PER 8}

\section{Basaltic andesite, S of Culiacán}

Long W Lat N

$-107.12 \quad 24.38$

laser step-heating experiments on groundmass sample

\begin{tabular}{|ccccccccccc|} 
Pwr & ${ }^{39} \mathrm{Ar} \times 10^{-6}$ & $\mathrm{~F}^{39} \mathrm{Ar}$ & ${ }^{40} \mathrm{Ar}^{*}{ }^{39} \mathrm{Ar}_{\mathrm{K}}$ & $1 \sigma$ & $\mathrm{Age}$ in $\mathrm{Ma}$ & $1 \sigma$ & & ${ }^{40}{ }^{40} \mathrm{Ar}^{*}$ & ${ }^{40} \mathrm{Ar}^{36} \mathrm{Ar}$ & ${ }^{37} \mathrm{Ar}_{\mathrm{Ca}}{ }^{39} \mathrm{Ar}_{\mathrm{K}}$ \\
\hline 0.40 & 412.642 & 0.0542 & 0.95 & 0.35 & 5.65 & 2.10 & $\mathrm{a}$ & 5.67 & 313.28 & 0.602 \\
0.80 & 762.979 & 0.1001 & 1.60 & 0.14 & 9.50 & 0.85 & $\mathrm{~b}$ & 21.27 & 375.31 & 1.173 \\
1.40 & 1705.234 & 0.2236 & 1.85 & 0.05 & 10.93 & 0.29 & $\mathrm{c}$ & 48.82 & 577.36 & 1.679 \\
3.00 & 2431.496 & 0.3188 & 1.90 & 0.03 & 11.25 & 0.17 & $\mathrm{~d}$ & 66.10 & 871.64 & 2.095 \\
7.00 & 2316.772 & 0.3033 & 1.78 & 0.03 & 10.52 & 0.20 & $\mathrm{e}$ & 54.41 & 648.11 & 4.195 \\
\hline
\end{tabular}

Integrated results

\begin{tabular}{|cccccccc|}
${ }^{39} \mathrm{Ar} \times 10^{-6}$ & ${ }^{40} \mathrm{Ar}^{*} /{ }^{39} \mathrm{Ar}_{\mathrm{K}}$ & $1 \sigma$ & Age in Ma & $1 \sigma$ & $\%{ }^{40} \mathrm{Ar}^{*}$ & ${ }^{40} \mathrm{Ar} /{ }^{36} \mathrm{Ar}$ & ${ }^{37} \mathrm{Ar}_{\mathrm{Ca}}{ }^{39} \mathrm{Ar}_{\mathrm{K}}$ \\
\hline 7617.0 & 1.77 & 0.03 & 10.48 & 0.19 & 40.07 & 493.07 & 2.466 \\
\hline
\end{tabular}

$\mathrm{J}=0.0032919 \pm 0.0000168$

Preferred age $t=10.94 \pm 0.23 \mathrm{Ma}$

calculated with the weighted mean of fractions c to e; $84.57 \%$ of ${ }^{39}$ Ar released in 3 consecutive fractions, MSWD = 3.74

$\mathrm{t}_{\mathrm{c}}=11.44 \pm 0.28 \mathrm{Ma},\left({ }^{40} \mathrm{Ar} /{ }^{36} \mathrm{Ar}\right)_{\mathrm{i}}=276 \pm 8, \mathrm{MSWD}=2.26$ for $\mathrm{n}=5$ 
SC 3

Santa Catalina Island andesite

Long W Lat N

$-110.79725 .683$

laser step-heating experiments on plagioclase concentrate

\begin{tabular}{|ccccccccccc|} 
Pwr & ${ }^{39} \mathrm{Ar} \times 10^{-6}$ & $\mathrm{~F}^{39} \mathrm{Ar}$ & ${ }^{40} \mathrm{Ar}^{*}{ }^{39} \mathrm{Ar}_{\mathrm{K}}$ & $1 \sigma$ & $\mathrm{Age}$ in Ma & $1 \sigma$ & & ${ }^{40} \mathrm{Ar}^{*}$ & ${ }^{40} \mathrm{Ar} /{ }^{36} \mathrm{Ar}$ & ${ }^{37} \mathrm{Ar}_{\mathrm{Ca}}{ }^{39} \mathrm{Ar}_{\mathrm{K}}$ \\
\hline 1.00 & 222.636 & 0.1262 & 5.83 & 0.70 & 33.19 & 3.92 & $\mathrm{a}$ & 13.21 & 340.47 & 4.168 \\
1.90 & 446.253 & 0.2530 & 2.84 & 0.19 & 16.22 & 1.10 & $\mathrm{~b}$ & 16.56 & 354.14 & 4.509 \\
3.00 & 565.409 & 0.3205 & 2.70 & 0.12 & 15.44 & 0.67 & $\mathrm{c}$ & 24.59 & 391.88 & 4.689 \\
4.00 & 234.360 & 0.1327 & 2.93 & 0.14 & 16.74 & 0.77 & $\mathrm{~d}$ & 28.60 & 413.86 & 6.739 \\
6.00 & 296.281 & 0.1676 & 3.42 & 0.13 & 19.52 & 0.75 & $\mathrm{e}$ & 23.45 & 386.03 & 7.915 \\
\hline 0.50 & 60.708 & 0.0845 & 4.41 & 0.74 & 25.17 & 4.22 & $\mathrm{f}$ & 8.60 & 323.29 & 2.785 \\
1.00 & 127.514 & 0.1772 & 2.39 & 0.35 & 13.66 & 1.98 & $\mathrm{~g}$ & 9.86 & 327.83 & 5.729 \\
1.60 & 101.989 & 0.1419 & 1.90 & 0.12 & 10.88 & 0.67 & $\mathrm{~h}$ & 19.96 & 369.21 & 4.011 \\
2.50 & 117.970 & 0.1642 & 1.90 & 0.14 & 10.90 & 0.82 & $\mathrm{i}$ & 18.08 & 360.71 & 3.623 \\
4.00 & 145.792 & 0.2025 & 2.95 & 0.13 & 16.84 & 0.76 & $\mathrm{j}$ & 21.92 & 378.44 & 7.077 \\
6.00 & 165.553 & 0.2297 & 3.92 & 0.28 & 22.38 & 1.56 & $\mathrm{k}$ & 22.93 & 383.41 & 8.488 \\
\hline
\end{tabular}

Integrated results

\begin{tabular}{|cccccccc|}
${ }^{39} \mathrm{Ar} \times 10^{-6}$ & ${ }^{40} \mathrm{Ar}^{*} /{ }^{39} \mathrm{Ar}_{\mathrm{K}}$ & $1 \sigma$ & Age in Ma & $1 \sigma$ & ${ }^{40}{ }^{40} \mathrm{Ar}^{*}$ & ${ }^{40} \mathrm{Ar}^{36} \mathrm{Ar}$ & ${ }^{37} \mathrm{Ar}_{\mathrm{Ca}}{ }^{39} \mathrm{Ar}_{\mathrm{K}}$ \\
\hline 1759.0 & 3.28 & 0.12 & 18.74 & 0.69 & 19.04 & 365.00 & 5.390 \\
\hline 716.8 & 2.87 & 0.13 & 16.44 & 0.72 & 15.66 & 350.38 & 5.797 \\
\hline
\end{tabular}

$\mathrm{J}=0.003185 \pm 0.000011$

\section{Preferred age $t=10.89 \pm 0.52 \mathrm{Ma}$}

Weighted mean age calculated with fractions $h$ and $i$, which define the base of the saddle. $30.61 \%$ of ${ }^{39} \mathrm{Ar}$ released in 2 consecutive fractions, MSWD $<0.01$

$\mathrm{t}_{\mathrm{c}}=10.14 \pm 2.59 \mathrm{Ma},\left({ }^{40} \mathrm{Ar} /{ }^{36} \mathrm{Ar}\right)_{\mathrm{i}}=322 \pm 13, \mathrm{MSWD}=8.4$ for $\mathrm{n}=11$ 


\section{PER 7}

\section{Basaltic andesite, $\mathrm{S}$ of Culiacán}

Long W Lat N

$-107.35 \quad 24.71$

laser step-heating experiments on groundmass sample

\begin{tabular}{|ccccccccccc|} 
Pwr & ${ }^{39} \mathrm{Ar} \times 10^{-6}$ & $\mathrm{~F}^{39} \mathrm{Ar}$ & ${ }^{40} \mathrm{Ar}^{*}{ }^{39} \mathrm{Ar}_{\mathrm{K}}$ & $1 \sigma$ & $\mathrm{Age}$ in Ma & $1 \sigma$ & & ${ }^{40} \mathrm{Ar}^{*}$ & ${ }^{40} \mathrm{Ar} /{ }^{36} \mathrm{Ar}$ & ${ }^{37} \mathrm{Ar}_{\mathrm{Ca}}{ }^{39} \mathrm{Ar}_{\mathrm{K}}$ \\
\hline 0.50 & 1037.845 & 0.1427 & 1.41 & 0.16 & 8.38 & 0.95 & $\mathrm{a}$ & 13.05 & 339.84 & 0.535 \\
1.20 & 1846.448 & 0.2538 & 1.79 & 0.07 & 10.57 & 0.44 & $\mathrm{~b}$ & 29.13 & 416.97 & 1.309 \\
2.40 & 2286.918 & 0.3142 & 1.86 & 0.07 & 11.03 & 0.43 & $\mathrm{c}$ & 37.79 & 474.97 & 1.963 \\
7.00 & 2108.358 & 0.2892 & 1.75 & 0.04 & 10.35 & 0.25 & $\mathrm{~d}$ & 36.66 & 466.55 & 4.387 \\
\hline 0.50 & 1346.814 & 0.2240 & 2.35 & 0.17 & 13.92 & 1.03 & $\mathrm{e}$ & 24.72 & 392.52 & 0.720 \\
1.10 & 2102.762 & 0.3495 & 1.78 & 0.05 & 10.55 & 0.30 & $\mathrm{f}$ & 33.23 & 442.57 & 1.540 \\
1.80 & 1258.608 & 0.2091 & 1.72 & 0.05 & 10.21 & 0.30 & $\mathrm{~g}$ & 38.75 & 482.48 & 2.516 \\
3.80 & 922.477 & 0.1530 & 1.58 & 0.06 & 9.33 & 0.34 & $\mathrm{~h}$ & 35.11 & 455.38 & 5.201 \\
7.00 & 388.459 & 0.0644 & 1.88 & 0.14 & 11.10 & 0.81 & $\mathrm{i}$ & 40.51 & 496.76 & 6.323 \\
\hline
\end{tabular}

Integrated results

\begin{tabular}{|cccccccc|}
${ }^{39} \mathrm{Ar} \times 10^{-6}$ & ${ }^{40} \mathrm{Ar}^{*} /{ }^{39} \mathrm{Ar}_{\mathrm{K}}$ & $1 \sigma$ & Age in $\mathrm{Ma}$ & $1 \sigma$ & $\%{ }^{40} \mathrm{Ar} *$ & ${ }^{40} \mathrm{Ar} /{ }^{36} \mathrm{Ar}$ & ${ }^{37} \mathrm{Ar}_{\mathrm{Ca}}{ }^{39} \mathrm{Ar}_{\mathrm{K}}$ \\
\hline 7269.0 & 1.75 & 0.04 & 10.34 & 0.25 & 28.95 & 415.91 & 2.294 \\
\hline 6010.0 & 1.87 & 0.05 & 11.08 & 0.29 & 31.62 & 432.12 & 2.428 \\
\hline
\end{tabular}

$\mathrm{J}=0.0032919 \pm 0.0000168$

\section{Preferred age $t_{p}=10.54 \pm 0.20 \mathrm{Ma}$}

the plateau age was calculated with the weighted mean of fractions b to d; $86.73 \%$ of ${ }^{39} \mathrm{Ar}$ released in 3 consecutive fractions, MSWD $=0.93$

$\mathrm{t}_{\mathrm{c}}=9.76 \pm 0.90 \mathrm{Ma},\left({ }^{40} \mathrm{Ar} /{ }^{36} \mathrm{Ar}\right)_{\mathrm{i}}=304 \pm 13, \mathrm{MSWD}=3.57$ for $\mathrm{n}=9$ 


\section{HUA 6}

\section{Basaltic andesite, Road to Las Peñitas microwave st}

\section{Long W Lat N}

$-105.22 \quad 21.94$

laser step-heating experiments on whole rock sample

\begin{tabular}{|c|c|c|c|c|c|c|c|c|c|c|c|}
\hline Pwr & ${ }^{39} \mathrm{Ar} \times 10^{-6}$ & $\mathrm{~F}^{39} \mathrm{Ar}$ & ${ }^{40} \mathrm{Ar}^{*} /{ }^{39} \mathrm{Ar}_{\mathrm{K}}$ & $1 \sigma$ & Age in $\mathrm{Ma}$ & $1 \sigma$ & & & ${ }^{0}{ }^{40} \mathrm{Ar} *$ & ${ }^{40} \mathrm{Ar} /{ }^{36} \mathrm{Ar}$ & ${ }^{37} \mathrm{Ar}_{\mathrm{Ca}}{ }^{39} \mathrm{Ar}_{\mathrm{K}}$ \\
\hline 0.40 & 39.84 & 0.0107 & -1.74 & 3.46 & -10.39 & 20.69 & $\mathrm{a}$ & & -1.77 & 290.36 & 5.387 \\
\hline 1.10 & 266.93 & 0.0717 & 1.18 & 0.16 & 6.98 & 0.97 & $\mathrm{~b}$ & & 18.84 & 364.08 & 4.968 \\
\hline 1.70 & 746.60 & 0.2005 & 2.45 & 0.08 & 14.52 & 0.48 & $\mathrm{c}$ & $t$ & 95.33 & 6323.27 & 3.051 \\
\hline 2.70 & 1302.03 & 0.3497 & 1.76 & 0.03 & 10.43 & 0.18 & d & & 78.42 & 1369.52 & 2.148 \\
\hline 3.90 & 738.48 & 0.1983 & 1.72 & 0.03 & 10.18 & 0.20 & $\mathrm{e}$ & & 80.75 & 1535.19 & 2.939 \\
\hline 6.00 & 629.82 & 0.1691 & 1.75 & 0.04 & 10.39 & 0.26 & $\mathrm{f}$ & & 80.71 & 1532.25 & 4.111 \\
\hline 1.00 & 402.09 & 0.0719 & 2.11 & 0.59 & 12.49 & 3.46 & $\mathrm{~g}$ & & 10.73 & 331.02 & 5.033 \\
\hline 1.50 & 752.26 & 0.1344 & 1.71 & 0.04 & 10.10 & 0.23 & $\mathrm{~h}$ & & 59.36 & 727.06 & 3.511 \\
\hline 2.50 & 2004.26 & 0.3582 & 1.77 & 0.03 & 10.49 & 0.16 & $\mathrm{i}$ & & 75.84 & 1222.90 & 2.355 \\
\hline 3.50 & 972.17 & 0.1737 & 1.84 & 0.04 & 10.90 & 0.25 & $\mathrm{j}$ & & 87.00 & 2273.12 & 2.416 \\
\hline 4.50 & 475.07 & 0.0849 & 1.74 & 0.05 & 10.33 & 0.28 & $\mathrm{k}$ & & 84.18 & 1868.06 & 2.776 \\
\hline 6.00 & 989.38 & 0.1768 & 1.89 & 0.06 & 11.21 & 0.35 & 1 & & 91.12 & 3328.69 & 3.650 \\
\hline
\end{tabular}

Integrated results

\begin{tabular}{|cccccccc|}
${ }^{39} \mathrm{Ar} \times 10^{-6}$ & ${ }^{40} \mathrm{Ar}^{*} /{ }^{39} \mathrm{Ar}_{\mathrm{K}}$ & $1 \sigma$ & Age in $\mathrm{Ma}$ & $1 \sigma$ & ${ }^{0}{ }^{40} \mathrm{Ar}^{*}$ & ${ }^{40} \mathrm{Ar} /{ }^{36} \mathrm{Ar}$ & ${ }^{37} \mathrm{Ar}_{\mathrm{Ca}}{ }^{39} \mathrm{Ar}_{\mathrm{K}}$ \\
\hline 3724.0 & 1.81 & 0.05 & 10.73 & 0.27 & 50.39 & 595.68 & 3.055 \\
\hline 5595.0 & 1.82 & 0.05 & 10.77 & 0.28 & 51.26 & 606.30 & 2.978 \\
\hline
\end{tabular}

$\mathrm{J}=0.0032919 \pm 0.0000168$

\section{Preferred age $t_{p}=10.45 \pm 0.15 \mathrm{Ma}$,}

calculated with the weighted mean of fractions $\mathrm{h}$ to $\mathrm{k} ; 75.13 \%$ of ${ }^{39}$ Ar released in 4 consecutive fractions, MSWD = 1.95

$\mathrm{t}_{\mathrm{c}}=10.61 \pm 0.15 \mathrm{Ma},\left({ }^{40} \mathrm{Ar} /{ }^{36} \mathrm{Ar}\right)_{\mathrm{i}}=279 \pm 10, \mathrm{MSWD}=2.85$ for $\mathrm{n}=11$

$\$$ Fraction ignored in the isochron 


\section{PER 14}

\section{Basaltic andesite, $\mathrm{W}$ of Pericos VF}

Long W Lat N

$-108.00 \quad 25.15$

Ta furnace step-heating experiment on groundmass sample

\begin{tabular}{|c|c|c|c|c|c|c|c|c|c|c|}
\hline Temp ${ }^{\circ} \mathrm{C}$ & ${ }^{39} \mathrm{Ar} \mathrm{cc} \mathrm{STP} / \mathrm{g}$ & $\mathrm{F}^{39} \mathrm{Ar}$ & ${ }^{40} \mathrm{Ar}^{*} /{ }^{39} \mathrm{Ar}_{\mathrm{K}}$ & $1 \sigma$ & Age in $\mathrm{Ma}$ & $1 \sigma$ & & $\%{ }^{40} \mathrm{Ar}^{*}$ & ${ }^{40} \mathrm{Ar} /{ }^{36} \mathrm{Ar}$ & ${ }^{37} \mathrm{Ar}_{\mathrm{Ca}}{ }^{39} \mathrm{Ar}_{\mathrm{K}}$ \\
\hline 500 & $9.555 \mathrm{E}-09$ & 0.0208 & 9.50 & 3.92 & 60.83 & 24.68 & $\mathrm{a}$ & 4.29 & 308.75 & 0.489 \\
\hline 600 & $1.015 \mathrm{E}-07$ & 0.2205 & 2.47 & 0.20 & 16.00 & 1.30 & $\mathrm{~b}$ & 19.37 & 366.50 & 0.269 \\
\hline 700 & $1.103 \mathrm{E}-07$ & 0.2396 & 1.71 & 0.08 & 11.09 & 0.50 & $\mathrm{c}$ & 34.11 & 448.50 & 0.774 \\
\hline 800 & 1.094E-07 & 0.2377 & 1.37 & 0.06 & 8.91 & 0.39 & d & 38.78 & 482.65 & 2.287 \\
\hline 850 & 4.690E-07 & 0.1019 & 1.76 & 0.09 & 11.44 & 0.60 & e & 51.46 & 608.75 & 2.877 \\
\hline 900 & $1.815 \mathrm{E}-08$ & 0.0394 & 1.57 & 0.23 & 10.21 & 1.47 & $\mathrm{f}$ & 32.45 & 437.47 & 3.260 \\
\hline 1000 & $2.418 \mathrm{E}-08$ & 0.0525 & 1.55 & 0.19 & 10.08 & 1.24 & $\mathrm{~g}$ & 23.60 & 386.81 & 3.173 \\
\hline 1100 & 2.982E-08 & 0.0648 & 1.85 & 0.18 & 12.00 & 1.14 & $\mathrm{~h}$ & 23.96 & 388.63 & 3.524 \\
\hline 1350 & $1.051 \mathrm{E}-08$ & 0.0228 & 1.23 & 0.43 & 7.97 & 2.78 & $\mathrm{i}$ & 10.65 & 330.71 & 0.188 \\
\hline
\end{tabular}

Integrated results

\begin{tabular}{|cccccccc|}
${ }^{39} \mathrm{ArccSTP} / \mathrm{g}$ & ${ }^{40} \mathrm{Ar}^{*} /{ }^{39} \mathrm{Ar}_{\mathrm{K}}$ & $1 \sigma$ & Age in Ma & $1 \sigma$ & $\%{ }^{40} \mathrm{Ar}^{*}$ & ${ }^{40} \mathrm{Ar} /{ }^{36} \mathrm{Ar}$ & ${ }^{37} \mathrm{Ar}_{\mathrm{Ca}}{ }^{39} \mathrm{Ar}_{\mathrm{K}}$ \\
\hline $4.604 \mathrm{E}-07$ & 1.95 & 0.18 & 12.64 & 1.19 & 17.56 & 358.44 & 2.076 \\
\hline
\end{tabular}

$\mathrm{J}=0.0035828 \pm 0.0000775$

sample weight $=1.1410 \mathrm{~g} ;$ estimated $0.67 \% \mathrm{~K}$

Preferred age $\mathbf{t}_{\mathbf{c}}=\mathbf{1 0 . 3 3} \pm \mathbf{0 . 8 8} \mathrm{Ma},\left({ }^{40} \mathrm{Ar} /{ }^{36} \mathrm{Ar}\right)_{\mathrm{i}}=310 \pm 4, \mathrm{MSWD}=28$ for $\mathrm{n}=9$ 


\section{PER 1}

Hawaiite lava, Pericos Volcanic field

Long W Lat N

$-107.52 \quad 25.04$

laser step-heating experiments on groundmass sample

\begin{tabular}{|c|c|c|c|c|c|c|c|c|c|c|c|}
\hline Pwr & ${ }^{39} \mathrm{Ar} \times 10^{-6}$ & $\mathrm{~F}^{39} \mathrm{Ar}$ & ${ }^{40} \mathrm{Ar}^{*} /{ }^{39} \mathrm{Ar}_{\mathrm{K}}$ & $1 \sigma$ & Age in $\mathrm{Ka}$ & $1 \sigma$ & & & $\%{ }^{40} \mathrm{Ar}^{*}$ & ${ }^{40} \mathrm{Ar} /{ }^{36} \mathrm{Ar}$ & ${ }^{37} \mathrm{Ar}_{\mathrm{Ca}}{ }^{39} \mathrm{Ar}_{\mathrm{K}}$ \\
\hline 0.80 & 379.17 & 0.0606 & -0.08 & 0.15 & -434 & 839 & $\mathrm{a}$ & 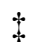 & -1.86 & 290.12 & 0.804 \\
\hline 1.50 & 1502.84 & 0.2401 & 0.16 & 0.02 & 890 & 122 & $\mathrm{~b}$ & & 34.98 & 454.50 & 0.585 \\
\hline 2.50 & 2436.37 & 0.3893 & 0.15 & 0.02 & 844 & 90 & $\mathrm{c}$ & & 36.85 & 467.92 & 0.760 \\
\hline 8.00 & 1939.88 & 0.3100 & 0.07 & 0.02 & 418 & 131 & d & $\ddagger$ & 8.17 & 321.78 & 4.138 \\
\hline 0.80 & 589.67 & 0.0410 & 4.52 & 0.36 & 25091 & 1974 & $\mathrm{e}$ & $t$ & 82.92 & 1730.48 & 0.926 \\
\hline 1.50 & 2814.98 & 0.1955 & 0.15 & 0.02 & 827 & 132 & $\mathrm{f}$ & & 32.22 & 436.00 & 0.623 \\
\hline 2.50 & 5074.98 & 0.3524 & 0.15 & 0.01 & 863 & 55 & $\mathrm{~g}$ & & 46.94 & 556.97 & 0.640 \\
\hline 8.05 & 5919.98 & 0.4111 & 0.12 & 0.02 & 656 & 107 & $\mathrm{~h}$ & $t$ & 21.62 & 377.01 & 2.188 \\
\hline
\end{tabular}

Integrated results

\begin{tabular}{|rccccccc|}
${ }^{39} \mathrm{Ar} \times 10^{-6}$ & ${ }^{40} \mathrm{Ar}^{*}{ }^{39} \mathrm{Ar}_{\mathrm{K}}$ & $1 \sigma$ & Age in $\mathrm{Ka}$ & $1 \sigma$ & ${ }^{0}{ }^{40} \mathrm{Ar}^{*}$ & ${ }^{40} \mathrm{Ar} /{ }^{36} \mathrm{Ar}$ & ${ }^{37} \mathrm{Ar}_{\mathrm{Ca}}{ }^{39} \mathrm{Ar}_{\mathrm{K}}$ \\
\hline 6258.0 & 0.12 & 0.01 & 646 & 80 & 14.33 & 344.94 & 1.768 \\
\hline 14400.0 & 0.32 & 0.02 & 1769 & 102 & 48.57 & 574.52 & 1.285 \\
\hline
\end{tabular}

$\mathrm{J}=0.0030998 \pm 0.000017$

$\mathrm{t}=857 \pm 51 \mathrm{Ka}$, calculated with the weighted mean of fractions $\mathrm{f}$ and $\mathrm{g} ; 54.79 \%$ of ${ }^{39} \mathrm{Ar}$ released in 2 consecutive fractions, MSWD $=0.06$

Preferred age $\mathbf{t}_{\mathbf{c}}=\mathbf{8 8 4} \pm \mathbf{9 7} \mathbf{K a},\left({ }^{40} \mathrm{Ar} /{ }^{36} \mathrm{Ar}\right)_{\mathrm{i}}=288 \pm 23, \mathrm{MSWD}=0.14$ for $\mathrm{n}=4$

\$ Fractions ignored in the isochron 


\section{PER 4}

Hawaiite lava, Pericos Volcanic field

Long W Lat N

$-107.54 \quad 25.11$

laser step-heating experiments on groundmass sample

\begin{tabular}{|c|c|c|c|c|c|c|c|c|c|c|c|}
\hline Pwr & ${ }^{39} \mathrm{Ar} \times 10^{-6}$ & $\mathrm{~F}^{39} \mathrm{Ar}$ & ${ }^{40} \mathrm{Ar}^{*} /{ }^{39} \mathrm{Ar}_{\mathrm{K}}$ & $1 \sigma$ & Age in $\mathrm{Ka}$ & $1 \sigma$ & & & $\%{ }^{40} \mathrm{Ar} *$ & ${ }^{40} \mathrm{Ar} /{ }^{36} \mathrm{Ar}$ & ${ }^{37} \mathrm{Ar}_{\mathrm{Ca}}{ }^{39} \mathrm{Ar}_{\mathrm{K}}$ \\
\hline 0.70 & 299.98 & 0.0642 & -0.42 & 0.14 & -2334 & 780 & $\mathrm{a}$ & 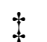 & -10.23 & 268.08 & 1.511 \\
\hline 1.20 & 671.32 & 0.1438 & 0.17 & 0.04 & 926 & 219 & $\mathrm{~b}$ & & 30.91 & 427.68 & 1.135 \\
\hline 2.00 & 420.10 & 0.0900 & 0.26 & 0.04 & 1447 & 207 & $\mathrm{c}$ & & 59.36 & 727.06 & 0.874 \\
\hline 5.00 & 2701.74 & 0.5782 & 0.17 & 0.04 & 936 & 201 & $\mathrm{~d}$ & & 34.90 & 453.94 & 2.394 \\
\hline 8.00 & 578.43 & 0.1238 & 0.34 & 0.22 & 1913 & 1234 & $\mathrm{e}$ & & 1.98 & 301.46 & 2.591 \\
\hline 0.50 & 308.14 & 0.0245 & -0.45 & 0.14 & -2537 & 762 & $\mathrm{f}$ & $\ddagger$ & -5.54 & 279.99 & 1.716 \\
\hline 1.00 & 942.28 & 0.0750 & 0.06 & 0.04 & 332 & 238 & $\mathrm{~g}$ & 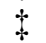 & 8.07 & 321.44 & 1.132 \\
\hline 2.00 & 1871.69 & 0.1490 & 0.13 & 0.03 & 754 & 164 & $\mathrm{~h}$ & & 31.04 & 428.50 & 1.032 \\
\hline 4.00 & 2487.30 & 0.1980 & 0.17 & 0.02 & 971 & 135 & $\mathrm{i}$ & & 50.62 & 598.43 & 1.624 \\
\hline 6.00 & 4754.56 & 0.3783 & 0.14 & 0.02 & 801 & 105 & $\mathrm{j}$ & & 43.35 & 521.60 & 2.298 \\
\hline 8.00 & 2202.06 & 0.1752 & ---- & ---- & ---- & ---- & $\mathrm{k}$ & & ---- & ---- & 2.501 \\
\hline
\end{tabular}

Integrated results

\begin{tabular}{|cccccccc|}
${ }^{39} \mathrm{Ar} \times 10^{-6}$ & ${ }^{40} \mathrm{Ar}^{*} /{ }^{39} \mathrm{Ar}_{\mathrm{K}}$ & $1 \sigma$ & $\mathrm{Age}$ in $\mathrm{Ka}$ & $1 \sigma$ & ${ }^{\%}{ }^{40} \mathrm{Ar} *$ & ${ }^{40} \mathrm{Ar}{ }^{36} \mathrm{Ar}$ & ${ }^{37} \mathrm{Ar}_{\mathrm{Ca}}{ }^{39} \mathrm{Ar}_{\mathrm{K}}$ \\
\hline 4665.0 & 0.16 & 0.04 & 891 & 209 & 5.70 & 313.36 & 2.04 \\
\hline 12550.0 & ---- & ---- & ---- & ---- & ---- & ---- & 1.91 \\
\hline
\end{tabular}

$\mathrm{J}=0.0030998 \pm 0.000017$

\section{Preferred age $t_{p}=843 \pm 74 \mathrm{Ka}$}

calculated with the weighted mean of fractions $h$ to $j ; 72.53 \%$ of ${ }^{39}$ Ar released in 3 consecutive fractions, MSWD $=0.68$

$\mathrm{t}_{\mathrm{c}}=853 \pm 80 \mathrm{Ka},\left({ }^{40} \mathrm{Ar} /{ }^{36} \mathrm{Ar}\right)_{\mathrm{i}}=300 \pm 9, \mathrm{MSWD}=2.56$ for $\mathrm{n}=7$

\$ Fractions ignored in the isochron

for fraction $\mathrm{k}$ mass 36 was not tuned properly, no age information can be obtained. 


\section{PER 6}

\section{Hawaiite lava, Pericos Volcanic field}

laser step-heating experiments on groundmass sample

\begin{tabular}{|c|c|c|c|c|c|c|c|c|c|c|c|}
\hline Pwr & ${ }^{39} \mathrm{Ar} \times 10^{-6}$ & $\mathrm{~F}^{39} \mathrm{Ar}$ & ${ }^{40} \mathrm{Ar}^{*}{ }^{39} \mathrm{Ar}_{\mathrm{K}}$ & $1 \sigma$ & Age in $\mathrm{Ka}$ & $1 \sigma$ & & & $\%{ }^{40} \mathrm{Ar}^{*}$ & ${ }^{40} \mathrm{Ar} /{ }^{36} \mathrm{Ar}$ & ${ }^{37} \mathrm{Ar}_{\mathrm{Ca}}{ }^{39} \mathrm{Ar}_{\mathrm{K}}$ \\
\hline 0.10 & 17.47 & 0.0020 & 2.52 & 2.09 & 14025 & 11606 & $\mathrm{a}$ & & 1.57 & 300.21 & 1.724 \\
\hline 0.25 & 51.28 & 0.0059 & -2.05 & 1.14 & -11521 & 6417 & $\mathrm{~b}$ & & -3.16 & 286.44 & 1.159 \\
\hline 0.80 & 1159.98 & 0.1330 & -0.02 & 0.07 & -92 & 412 & c & & -0.29 & 294.64 & 0.875 \\
\hline 1.50 & 3851.78 & 0.4417 & 0.12 & 0.02 & 694 & 102 & d & & 14.22 & 344.47 & 0.740 \\
\hline 8.00 & 3645.13 & 0.4174 & 0.08 & 0.02 & 467 & 97 & $\mathrm{e}$ & & 7.91 & 320.90 & 2.959 \\
\hline 0.55 & 560.595 & 0.0555 & -0.03 & 0.24 & -172 & 1369 & $\mathrm{f}$ & & -0.12 & 295.15 & 0.972 \\
\hline 1.00 & 2278.277 & 0.2256 & 0.45 & 0.10 & 2496 & 574 & $\mathrm{~g}$ & \$ & 32.44 & 437.42 & 0.871 \\
\hline 1.70 & 2575.058 & 0.2550 & 0.08 & 0.03 & 442 & 166 & $\mathrm{~h}$ & & 10.72 & 331.00 & 0.825 \\
\hline 3.00 & 2553.623 & 0.2529 & 0.18 & 0.05 & 1021 & 256 & $\mathrm{i}$ & $t$ & 31.10 & 428.85 & 0.865 \\
\hline 7.00 & 2137.154 & 0.2111 & 0.06 & 0.04 & 363 & 204 & $\mathrm{j}$ & & 8.73 & 323.77 & 4.743 \\
\hline
\end{tabular}

Integrated results

\begin{tabular}{|cccccccc|}
${ }^{39} \mathrm{Ar} \times 10^{-6}$ & ${ }^{40} \mathrm{Ar}^{*}{ }^{39} \mathrm{Ar}_{\mathrm{K}}$ & $1 \sigma$ & Age in Ka & $1 \sigma$ & ${ }^{0}{ }^{40} \mathrm{Ar}^{*}$ & ${ }^{40} \mathrm{Ar} /{ }^{36} \mathrm{Ar}$ & ${ }^{37} \mathrm{Ar}_{\mathrm{Ca}}{ }^{39} \mathrm{Ar}_{\mathrm{K}}$ \\
\hline 8716.0 & 0.08 & 0.02 & 450 & 99 & 3.52 & 306.30 & 1.688 \\
\hline 10090.0 & 0.18 & 0.03 & 1001 & 176 & 7.99 & 321.16 & 1.680 \\
\hline
\end{tabular}

$\mathrm{J}=0.0030998 \pm 0.000017$

$\mathrm{t}=574 \pm 113 \mathrm{Ka}$

calculated with the weighted mean of fractions $d$ and e; $85.91 \%$ of ${ }^{39}$ Ar released in 2 consecutive fractions, MSWD $=2.59$

Preferred age $\mathbf{t}_{\mathbf{c}}=\mathbf{5 8 5} \pm \mathbf{8 2} \mathbf{K a},\left({ }^{40} \mathrm{Ar} /{ }^{36} \mathrm{Ar}\right)_{\mathrm{i}}=292 \pm 3, \mathrm{MSWD}=1.36$ for $\mathrm{n}=8$

\$ Fractions ignored in the isochron 


\section{$\mathrm{CHO} 4$}

Volcanic field Los Choix

Long $\mathrm{W}$ Lat $\mathrm{N}$

$-108.38926 .825$

Ta furnace step-heating experiment on groundmass sample

\begin{tabular}{|c|c|c|c|c|c|c|c|c|c|c|}
\hline Temp ${ }^{\circ} \mathrm{C}$ & ${ }^{39} \mathrm{Ar} \mathrm{cc}$ STP $/ \mathrm{g}$ & $\mathrm{F}^{39} \mathrm{Ar}$ & ${ }^{40} \mathrm{Ar}^{*} /{ }^{39} \mathrm{Ar}_{\mathrm{K}}$ & $1 \sigma$ & Age in $\mathrm{Ka}$ & $1 \sigma$ & & $\%{ }^{40} \mathrm{Ar}^{*}$ & ${ }^{40} \mathrm{Ar} /{ }^{36} \mathrm{Ar}$ & ${ }^{37} \mathrm{Ar}_{\mathrm{Ca}}{ }^{39} \mathrm{Ar}_{\mathrm{K}}$ \\
\hline 350 & $3.5668 \mathrm{E}-09$ & 0.0041 & 5.59 & 2.78 & 36044 & 17741 & $\mathrm{a}$ & 6.05 & 314.52 & 0.931 \\
\hline 600 & $4.2325 \mathrm{E}-08$ & 0.0487 & -0.06 & 0.18 & -368 & 1172 & $\mathrm{~b}$ & -1.18 & 292.07 & 0.625 \\
\hline 1000 & $5.4710 \mathrm{E}-07$ & 0.6289 & 0.02 & 0.02 & 138 & 107 & $\mathrm{c}$ & 4.44 & 309.02 & 0.599 \\
\hline 1350 & $2.7698 \mathrm{E}-07$ & 0.3184 & 0.09 & 0.03 & 563 & 225 & $\mathrm{~d}$ & 9.11 & 325.11 & 3.600 \\
\hline
\end{tabular}

Integrated results

\begin{tabular}{|cccccccc|}
${ }^{39} \mathrm{Ar} \operatorname{ccSTP} / \mathrm{g}$ & ${ }^{40} \mathrm{Ar}^{*} /{ }^{39} \mathrm{Ar}_{\mathrm{K}}$ & $1 \sigma$ & Age in $\mathrm{Ka}$ & $1 \sigma$ & $\%{ }^{40} \mathrm{Ar} *$ & ${ }^{40} \mathrm{Ar} /{ }^{36} \mathrm{Ar}$ & ${ }^{37} \mathrm{Ar}_{\mathrm{Ca}}{ }^{39} \mathrm{Ar}_{\mathrm{K}}$ \\
\hline $8.700 \mathrm{E}-07$ & 0.06 & 0.04 & 397 & 232 & 5.02 & 311.13 & 1.557 \\
\hline
\end{tabular}

$\mathrm{J}=0.003608 \pm 0.000078$

sample weight $=0.9834 \mathrm{~g}$; estimated $1.27 \% \mathrm{~K}$

$\mathrm{t}_{\mathrm{c}}=29 \pm 181 \mathrm{Ka},\left({ }^{40} \mathrm{Ar} /{ }^{36} \mathrm{Ar}\right)_{\mathrm{i}}=311 \pm 10, \mathrm{MSWD}=5.0$ for $\mathrm{n}=4$

Preferred age: $t=138 \pm 107 \mathrm{Ka}$ 

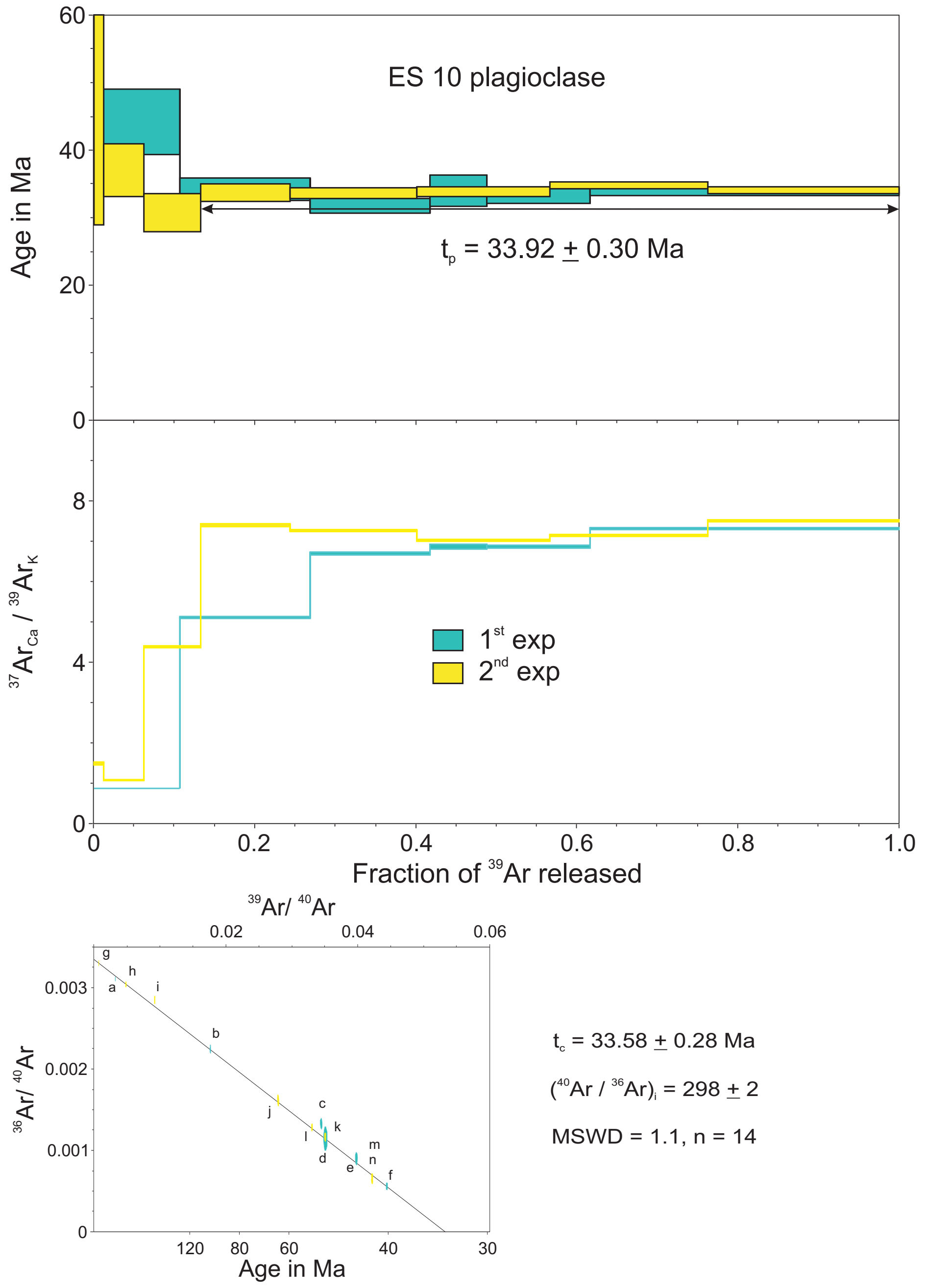

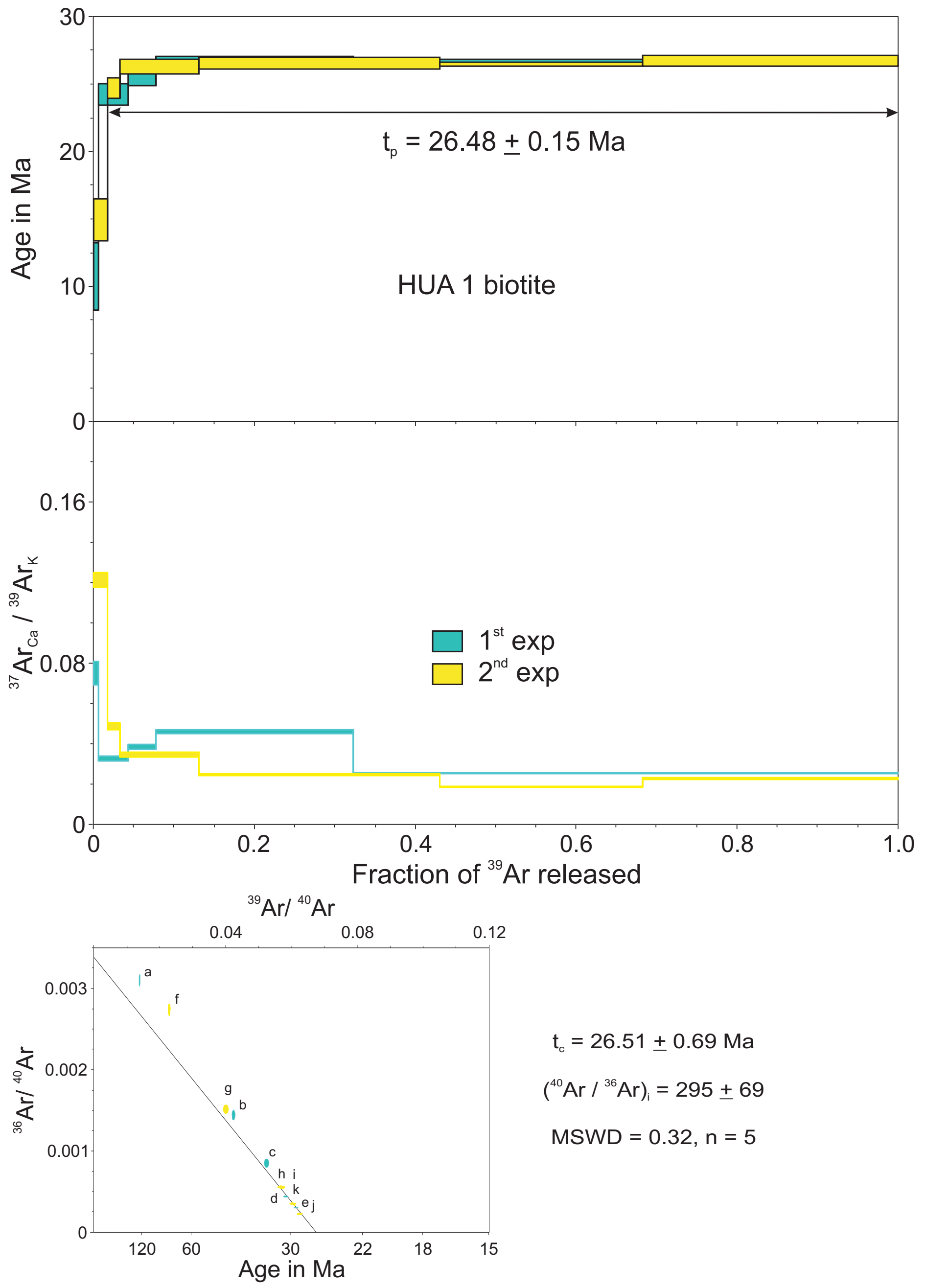


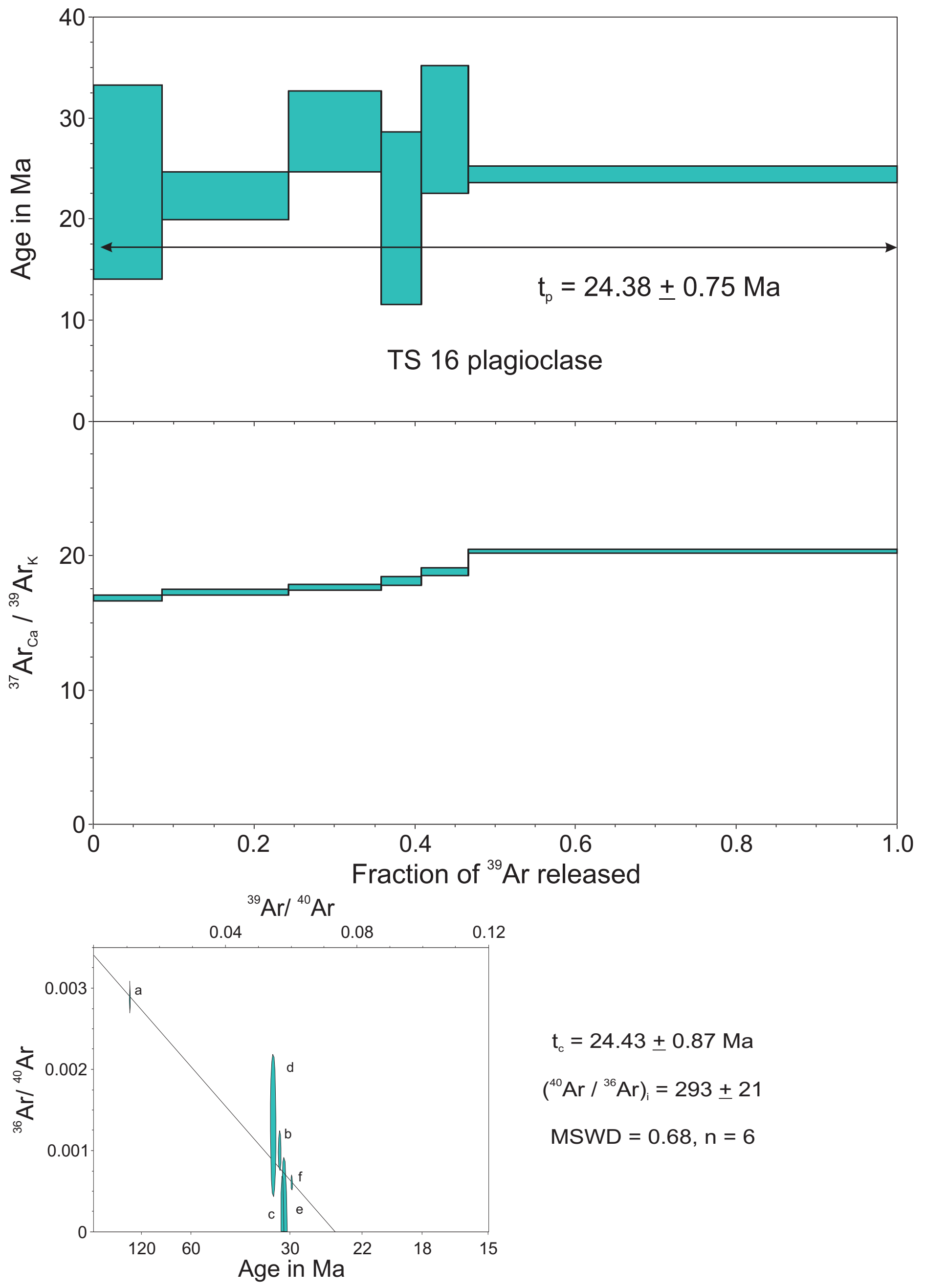




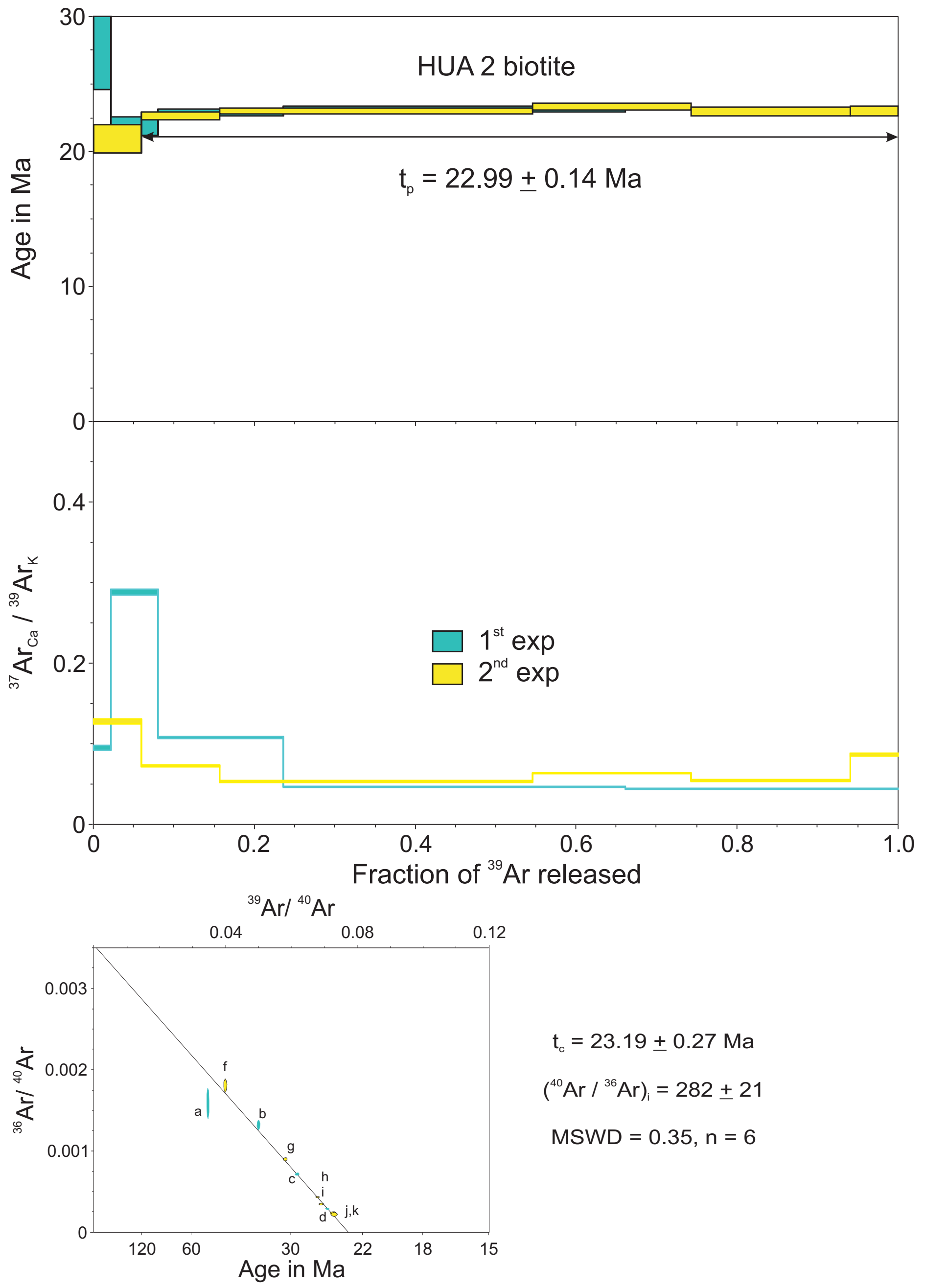



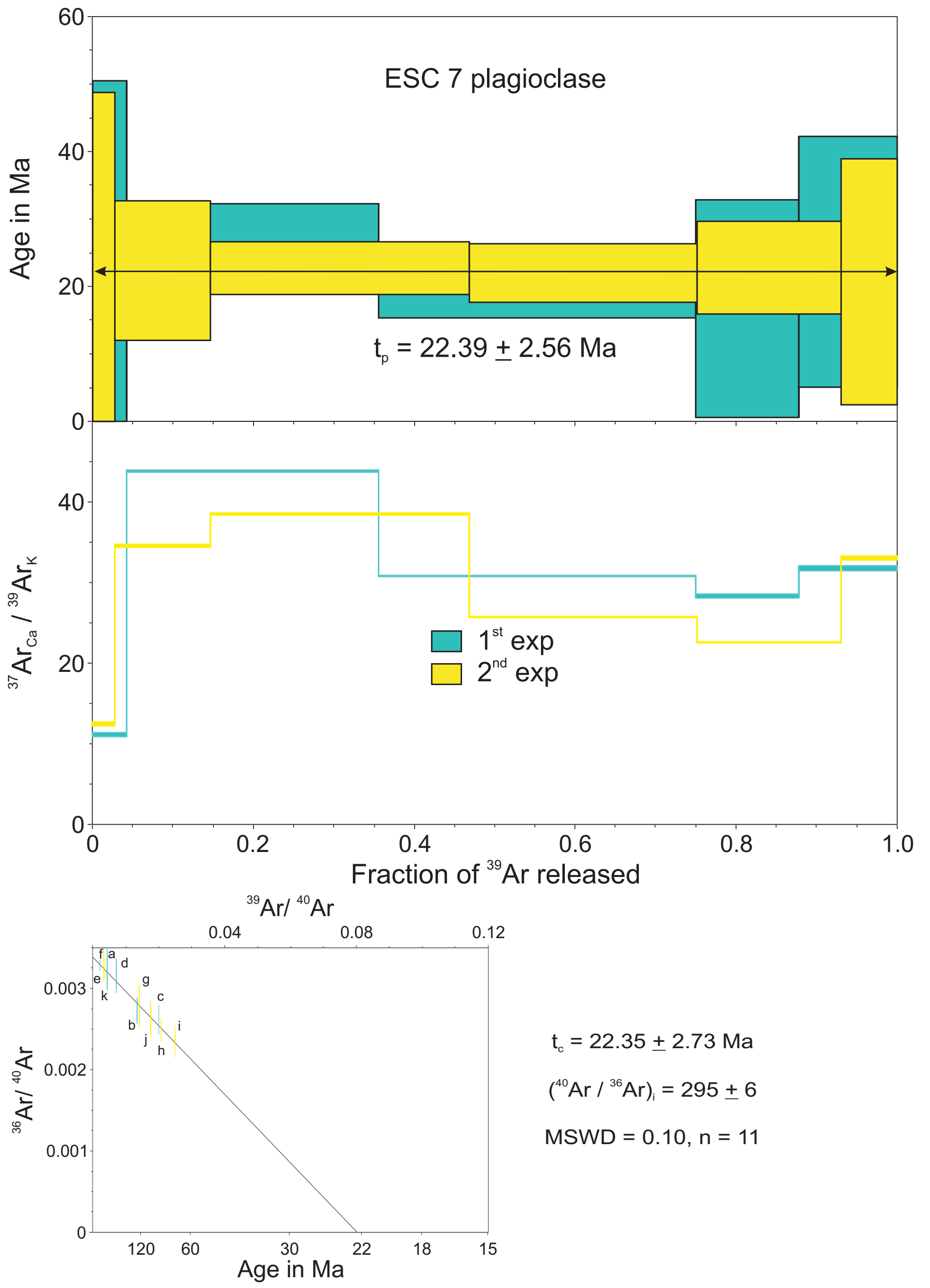

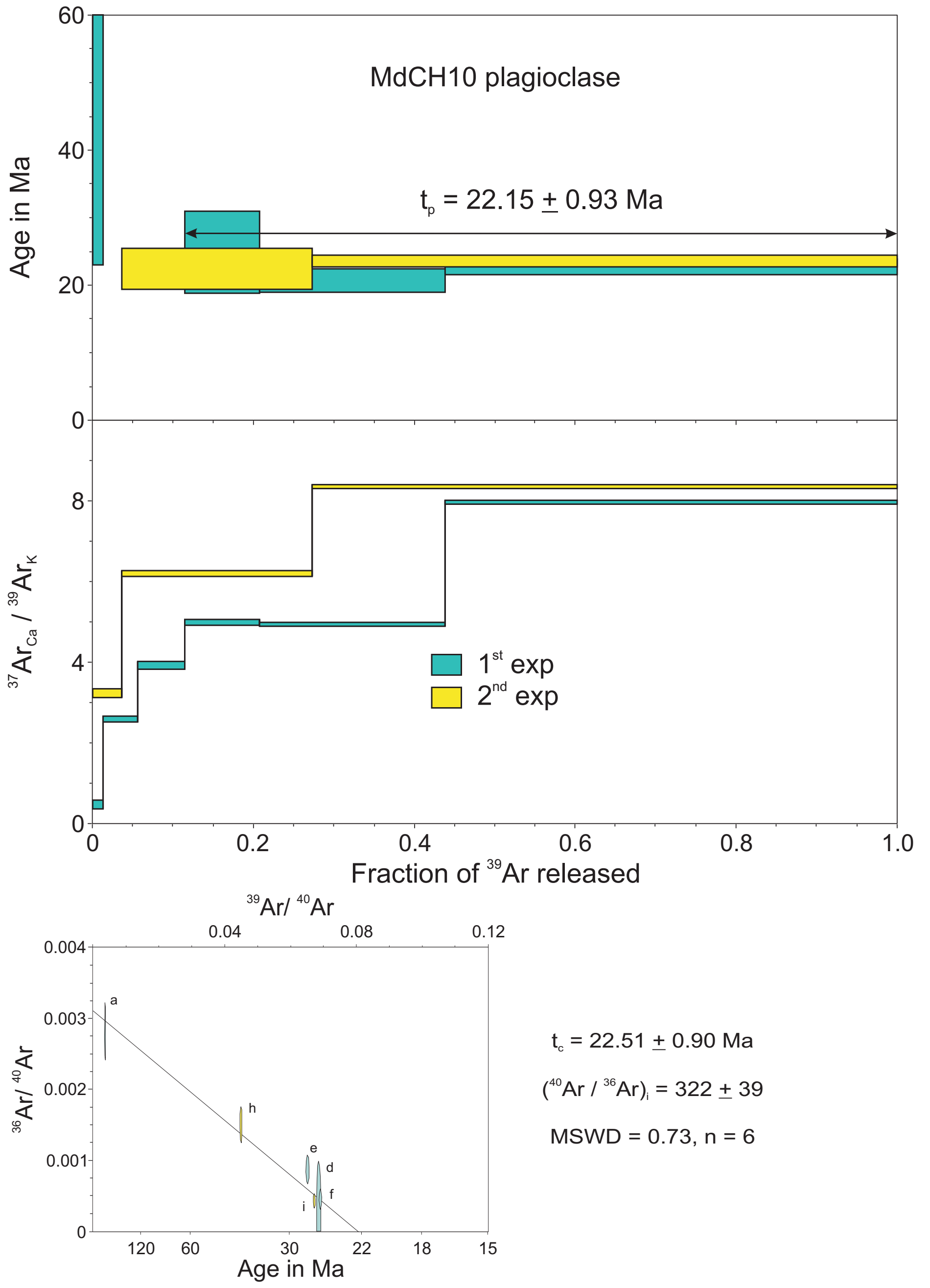

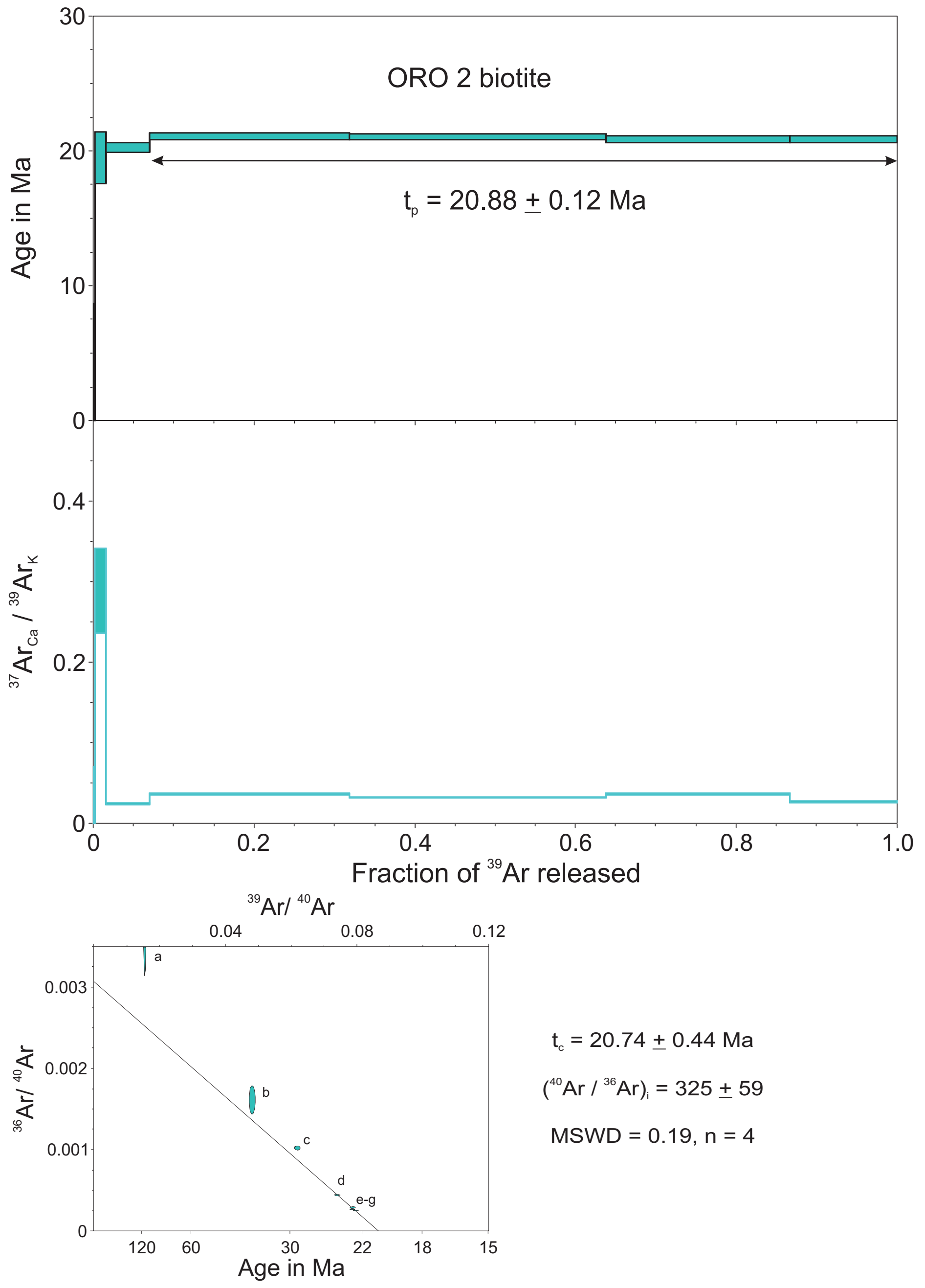

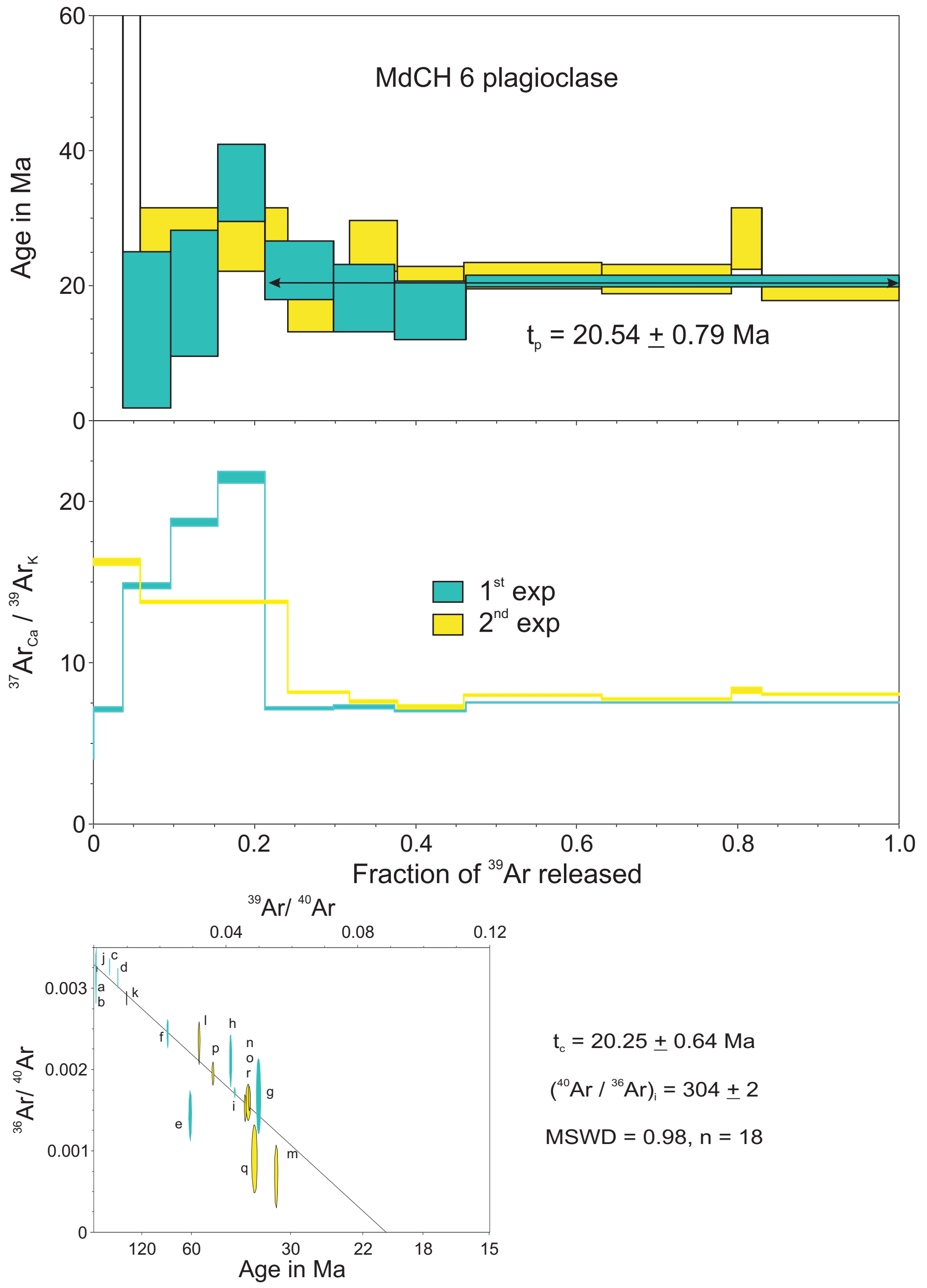

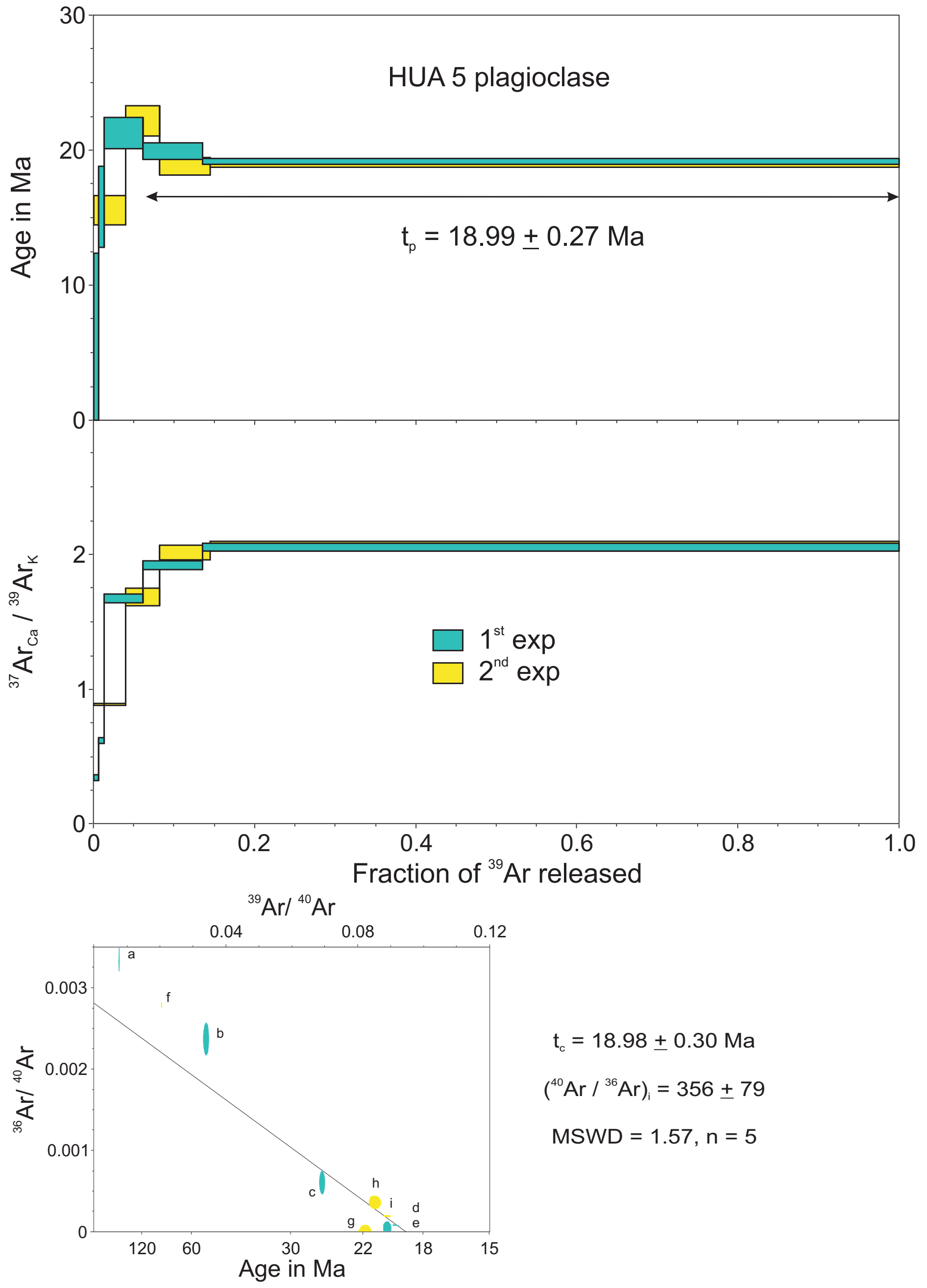

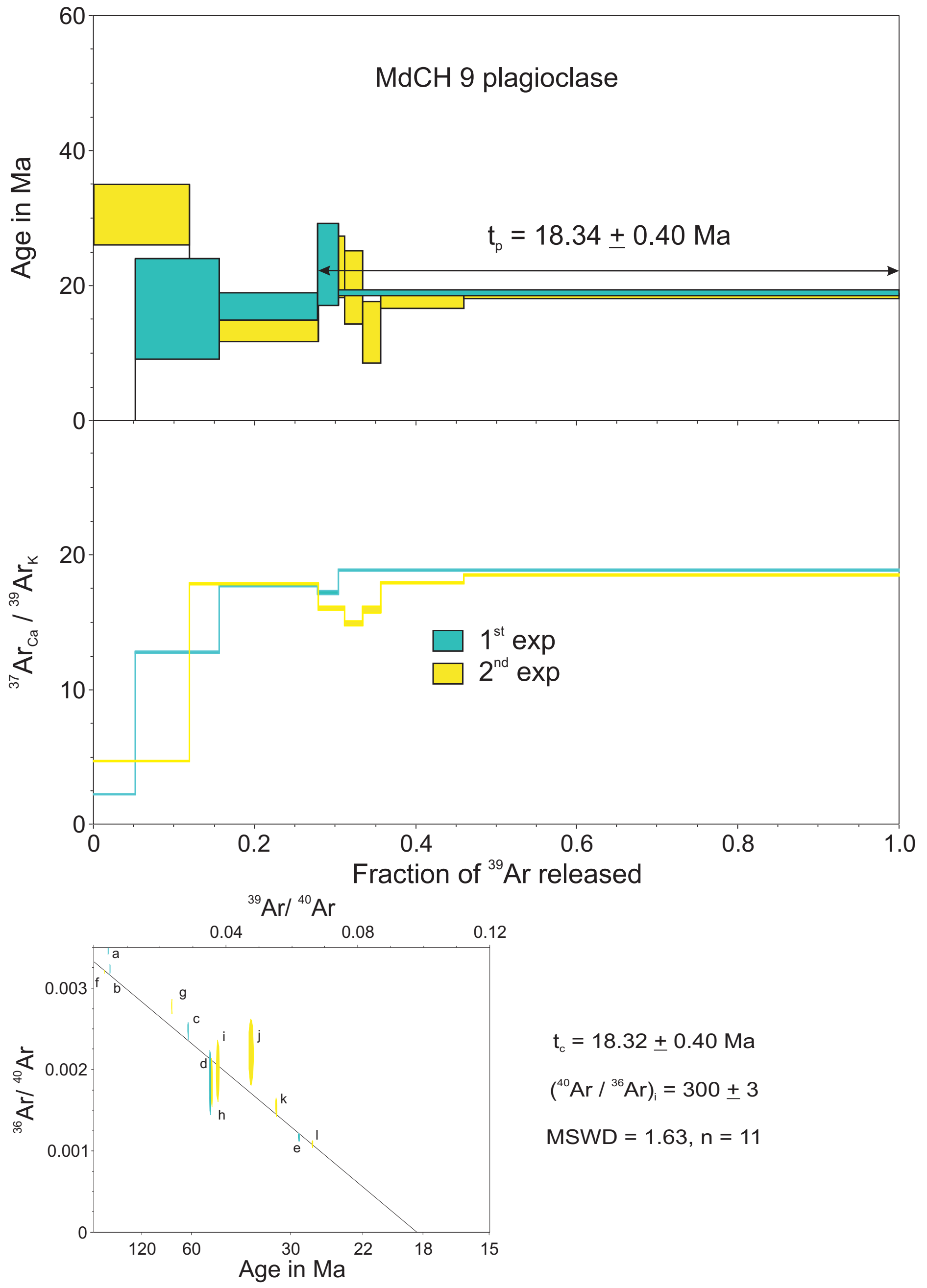


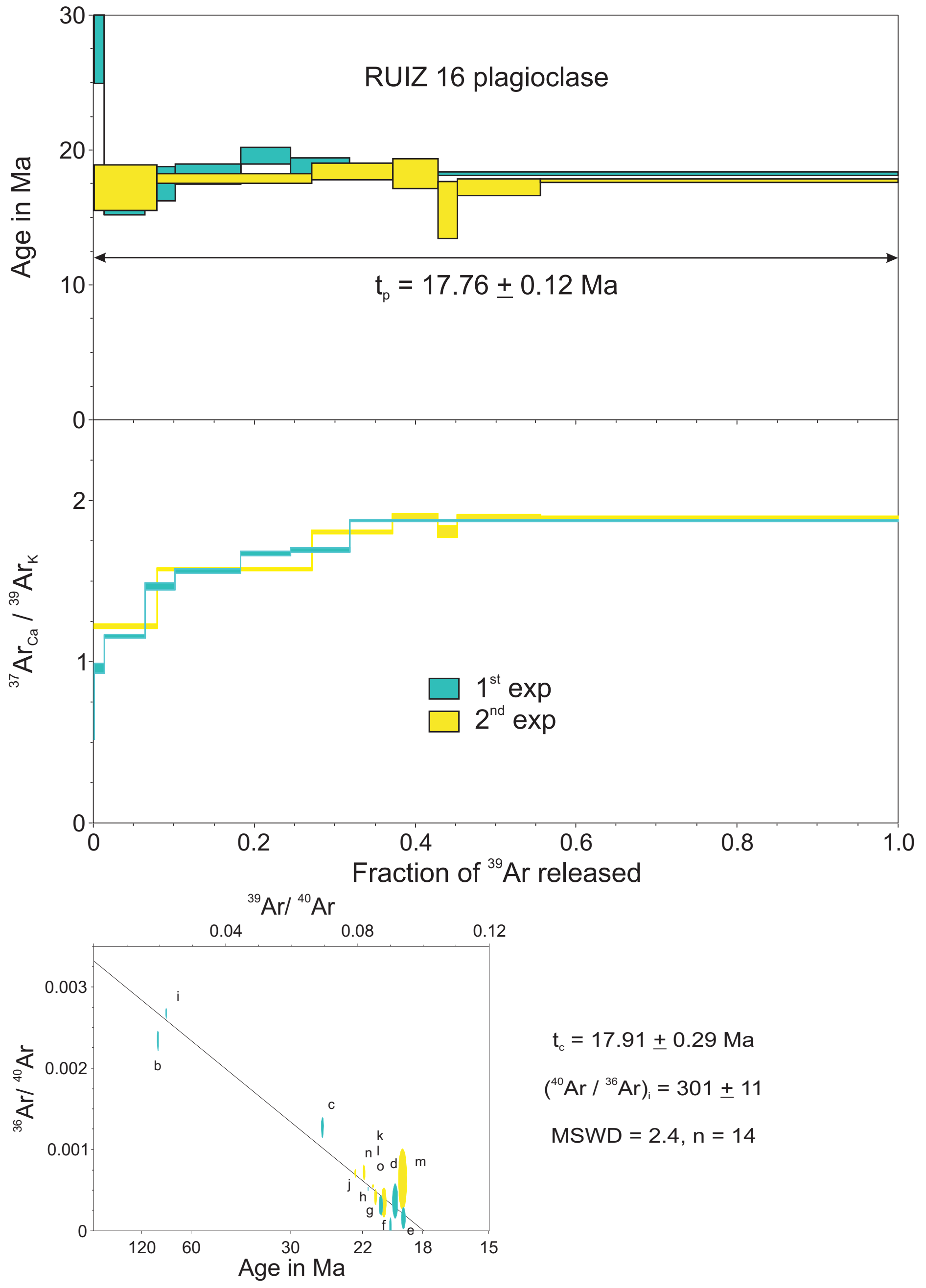




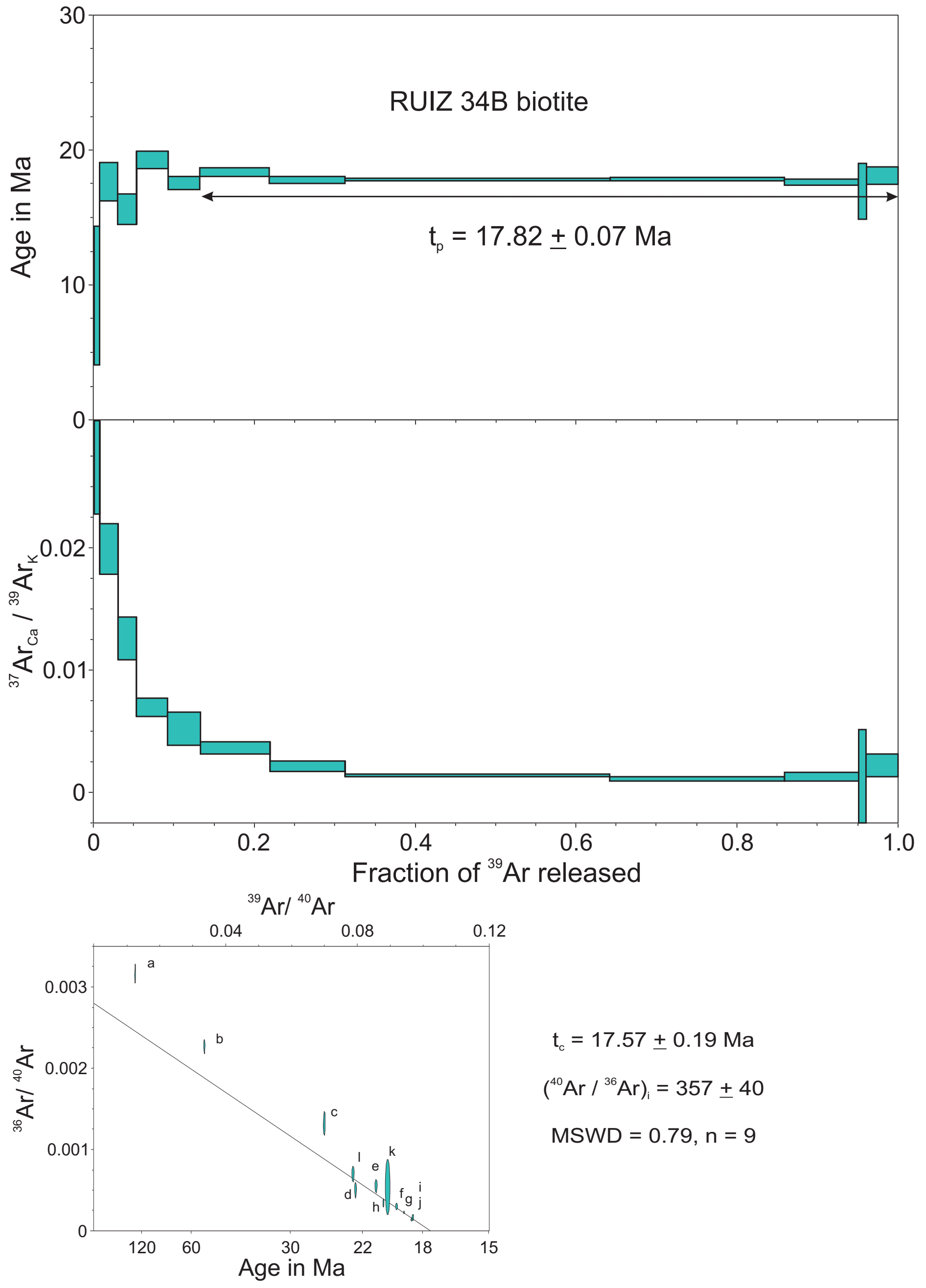



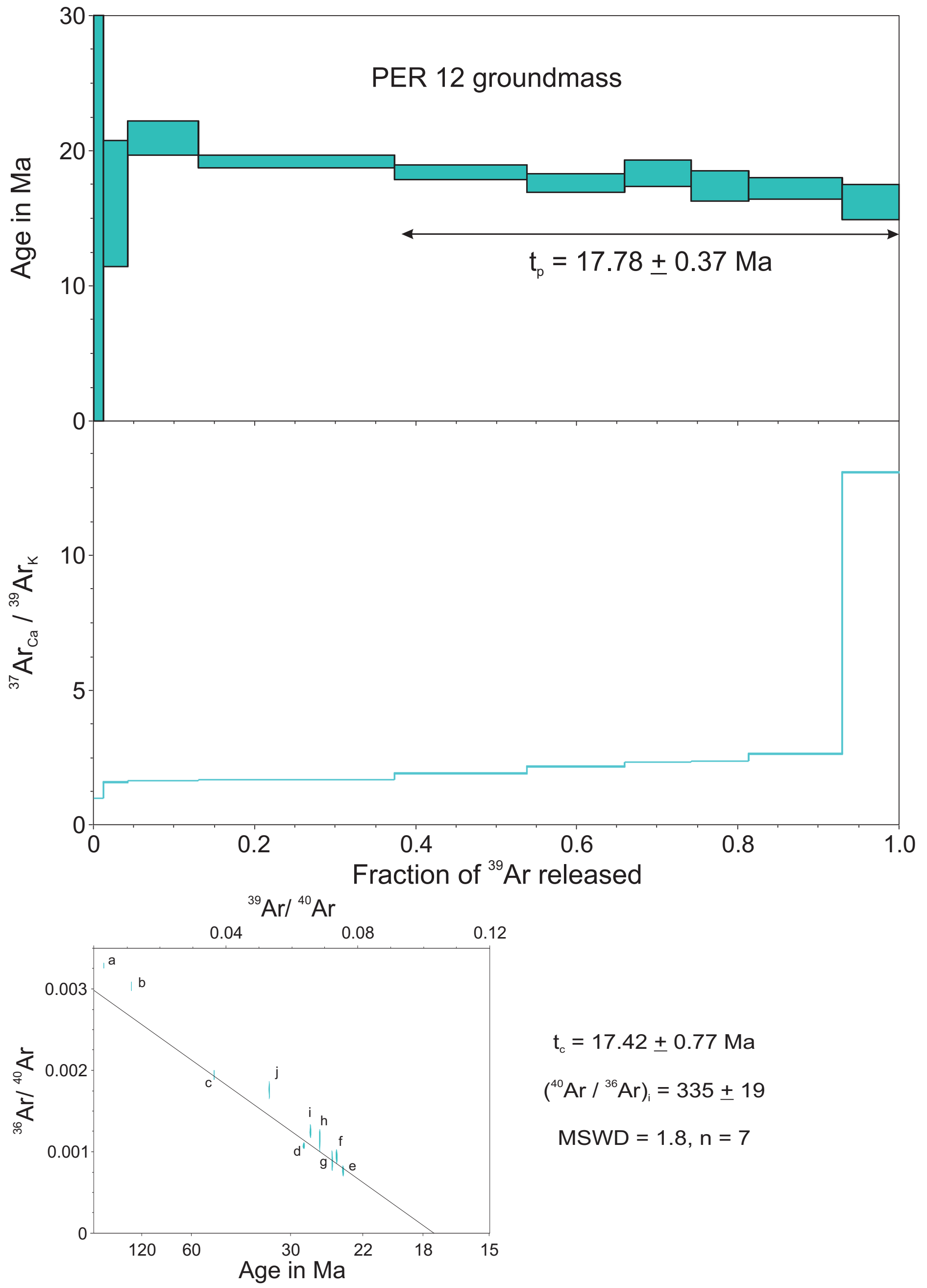


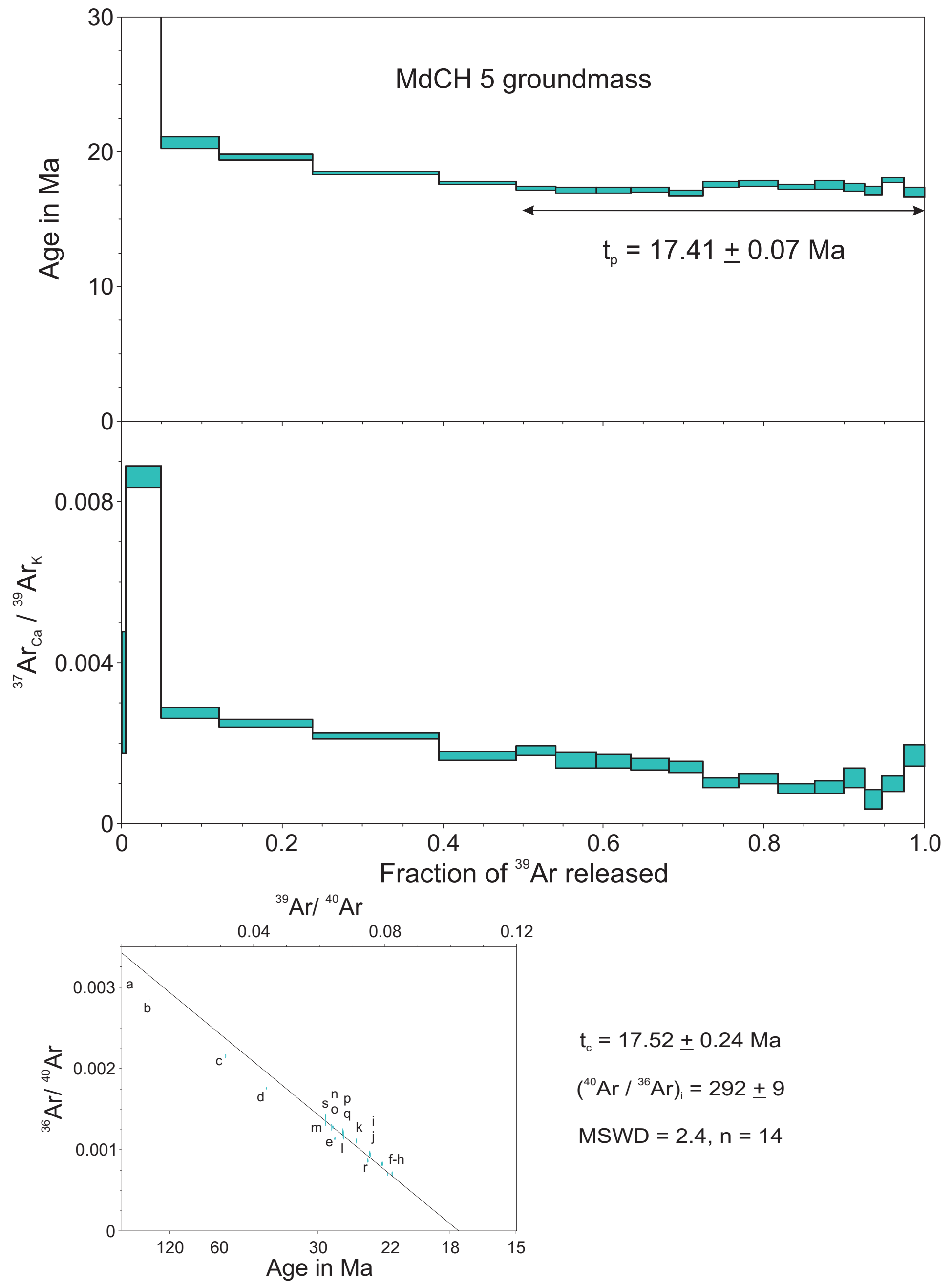



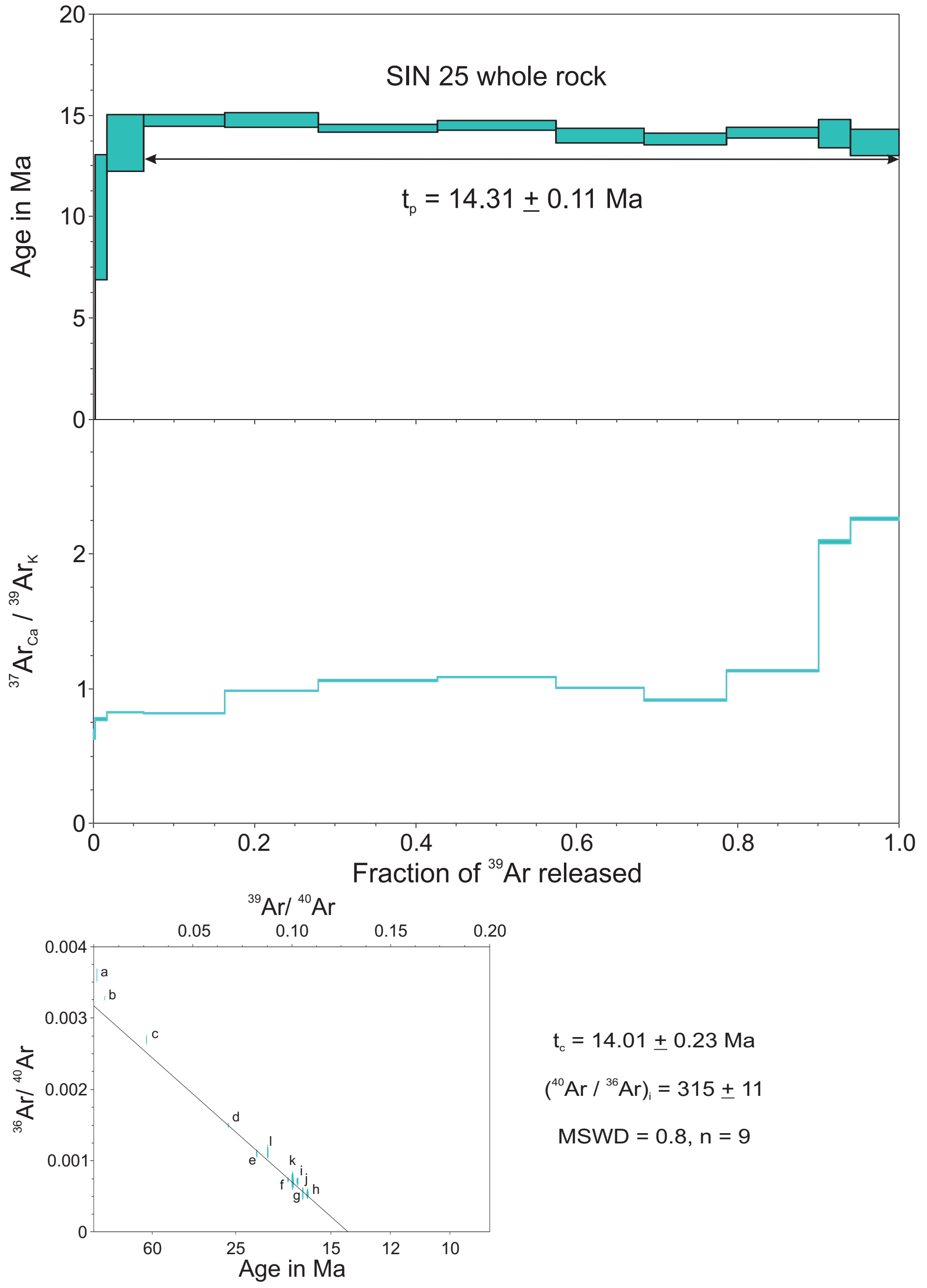

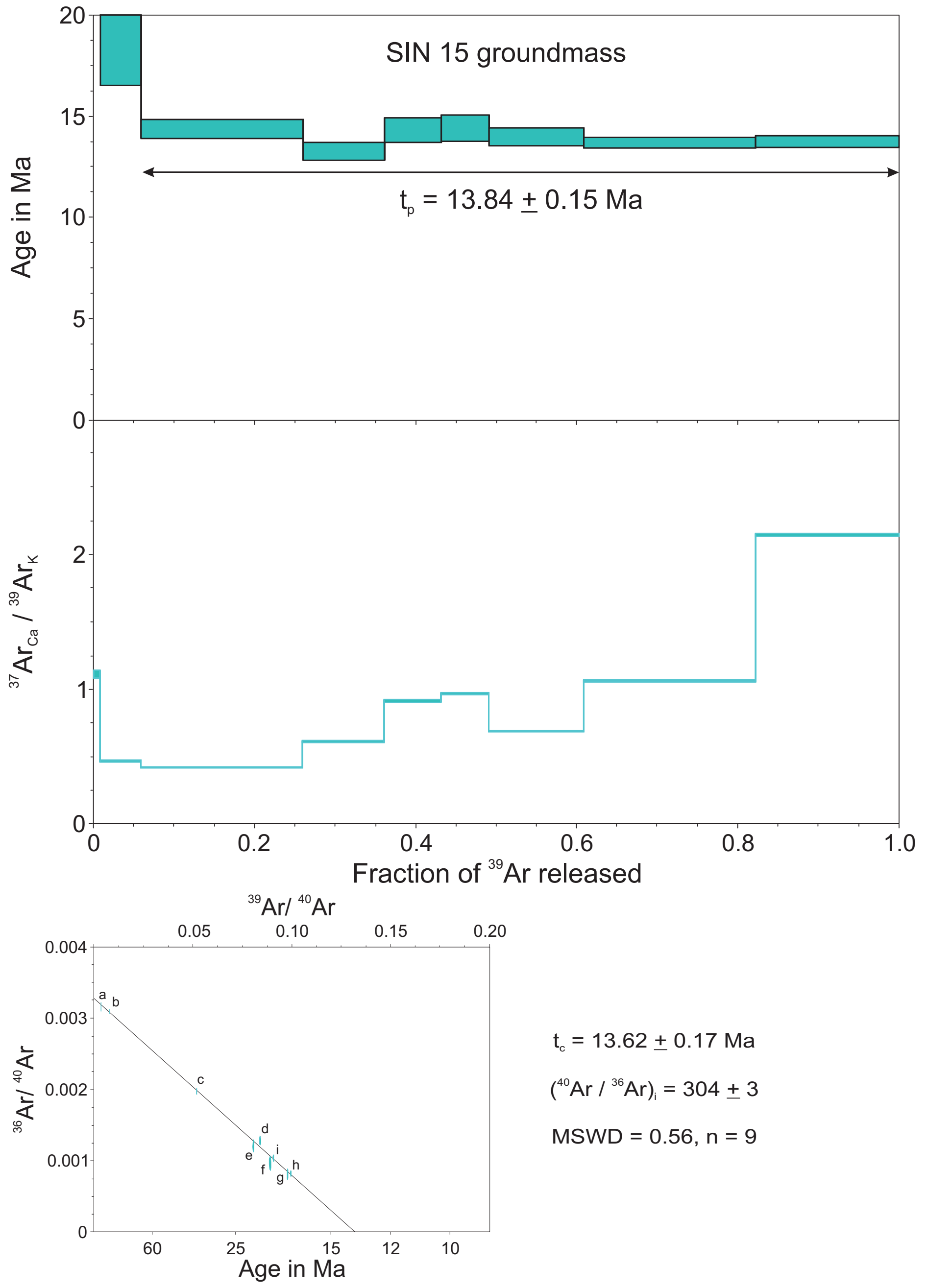


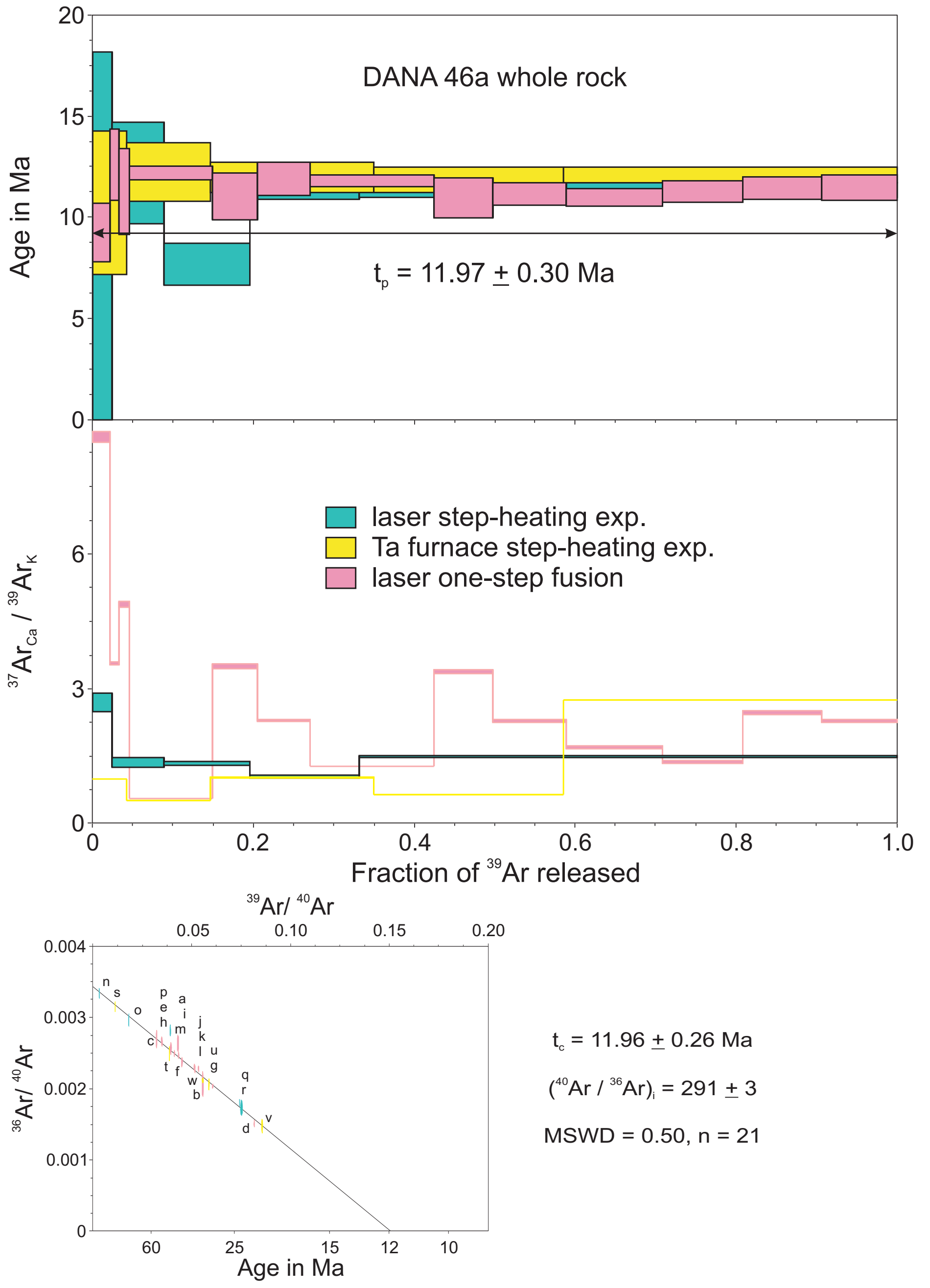




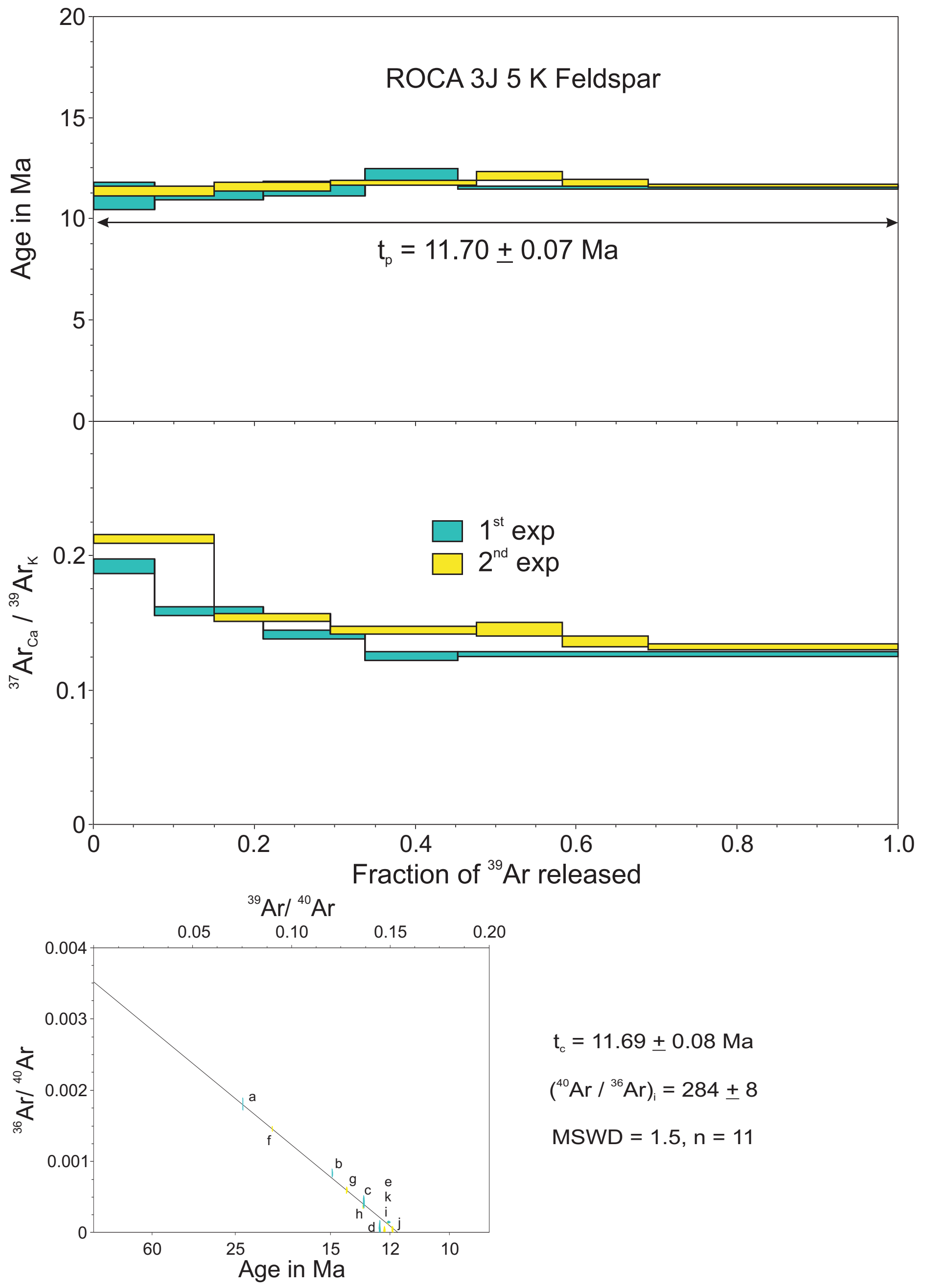



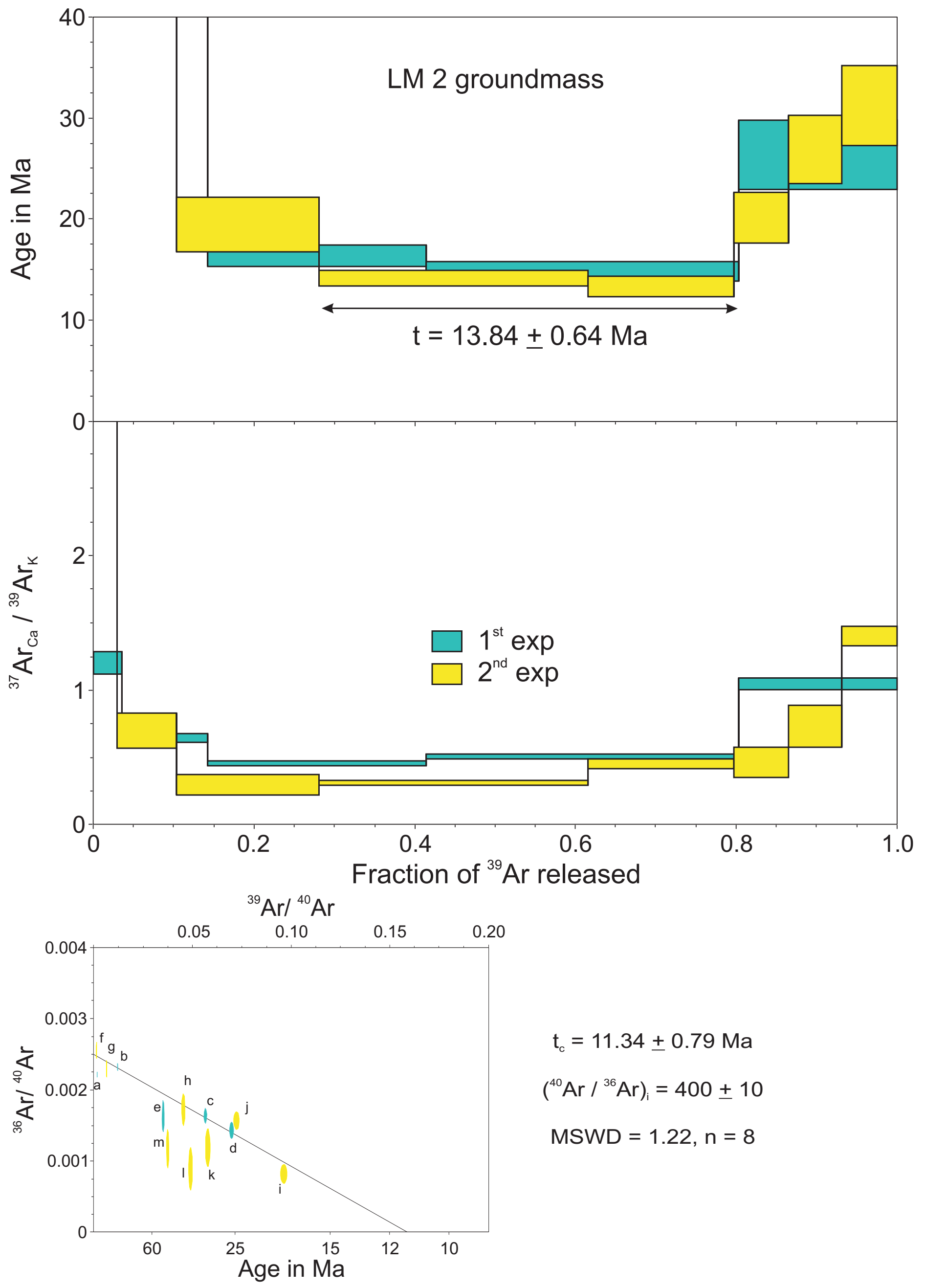

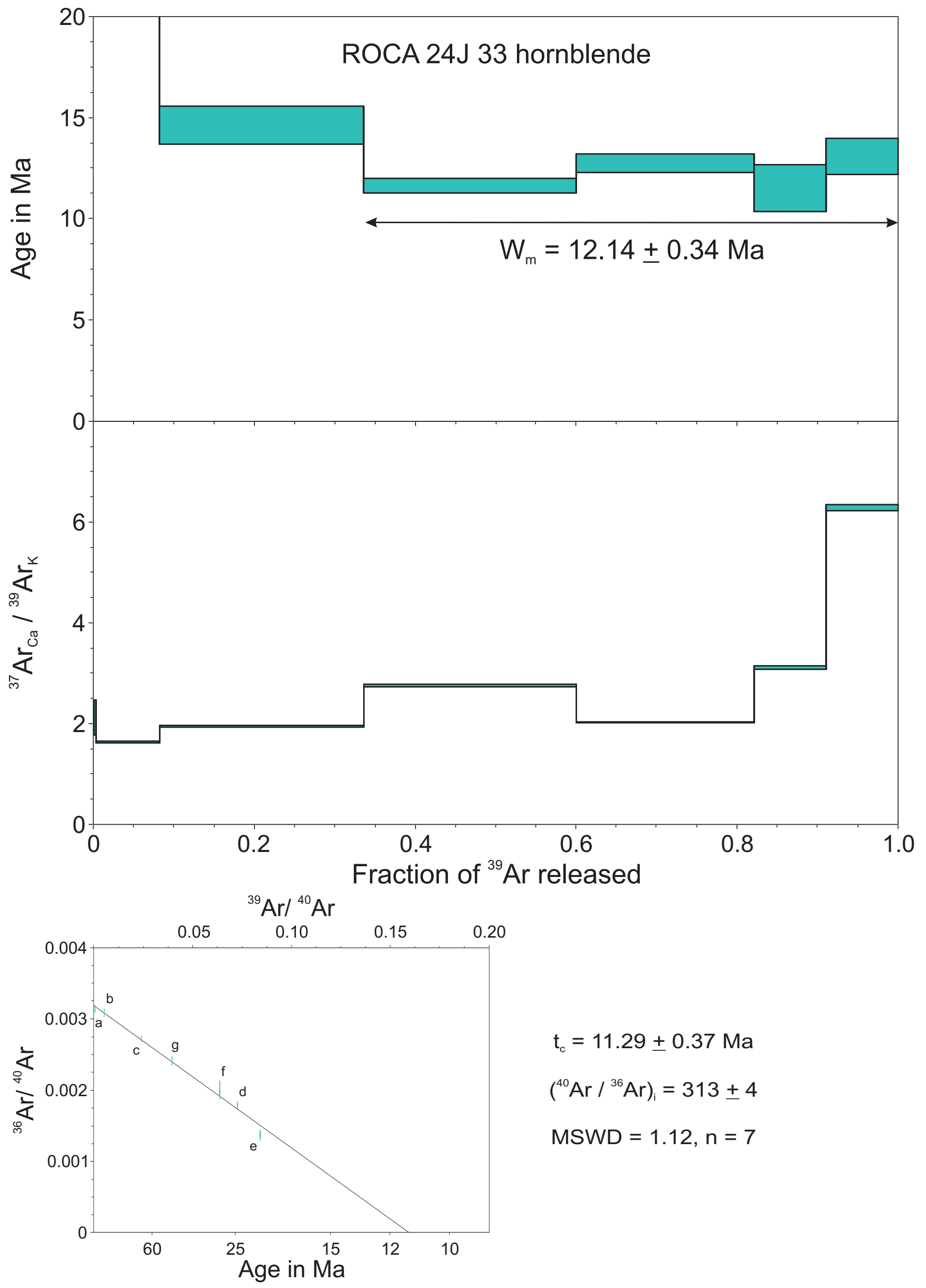

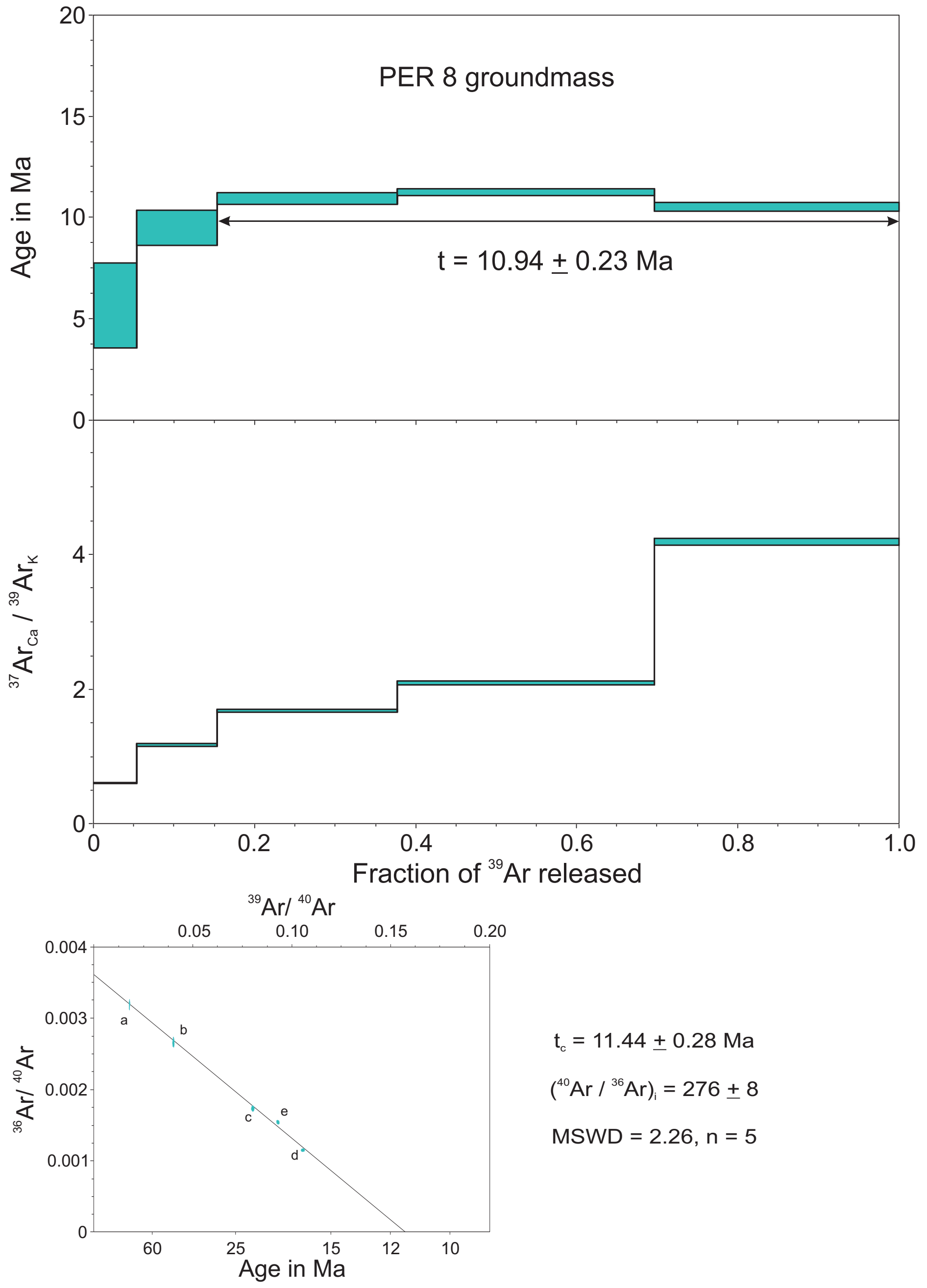

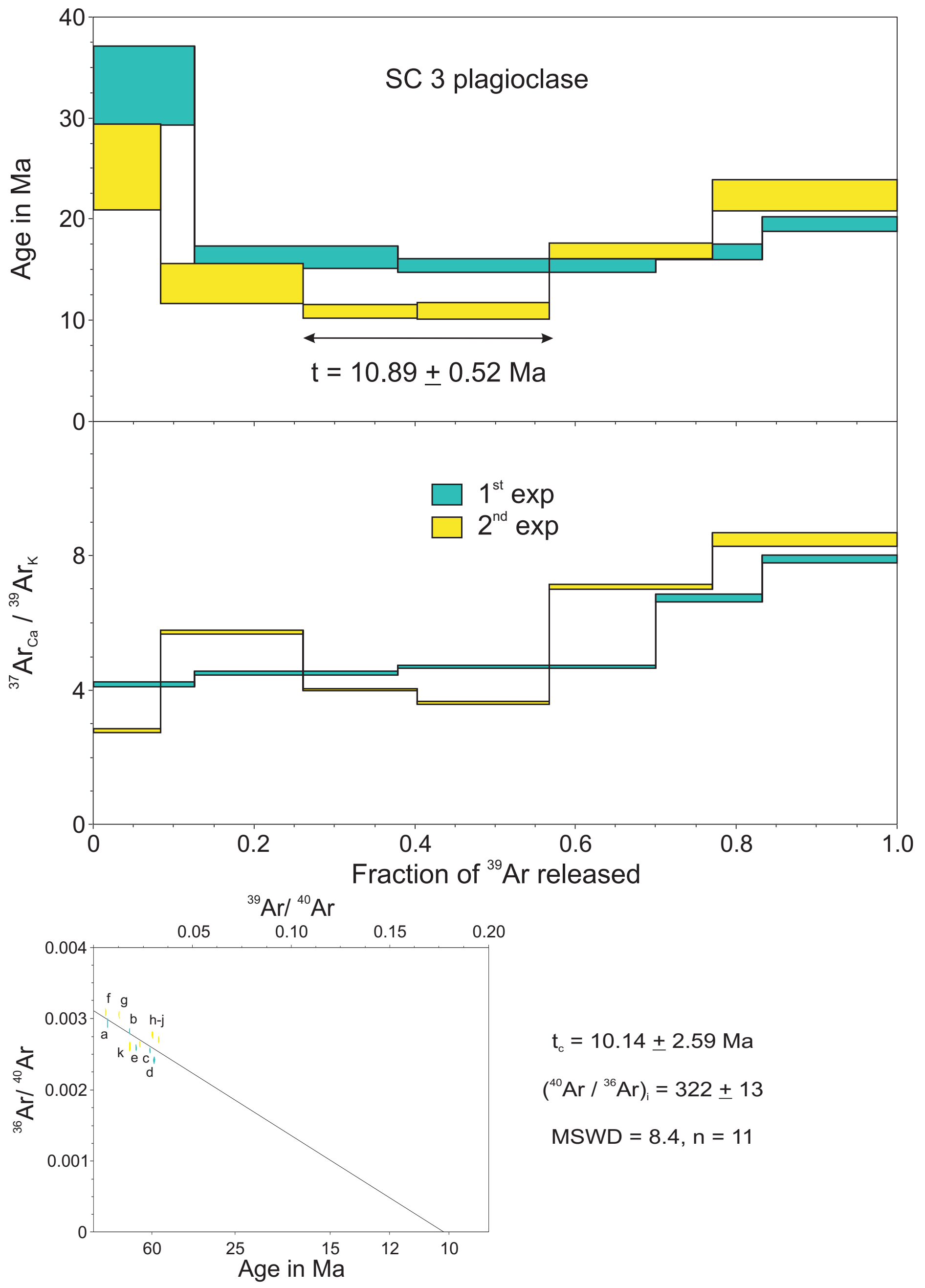

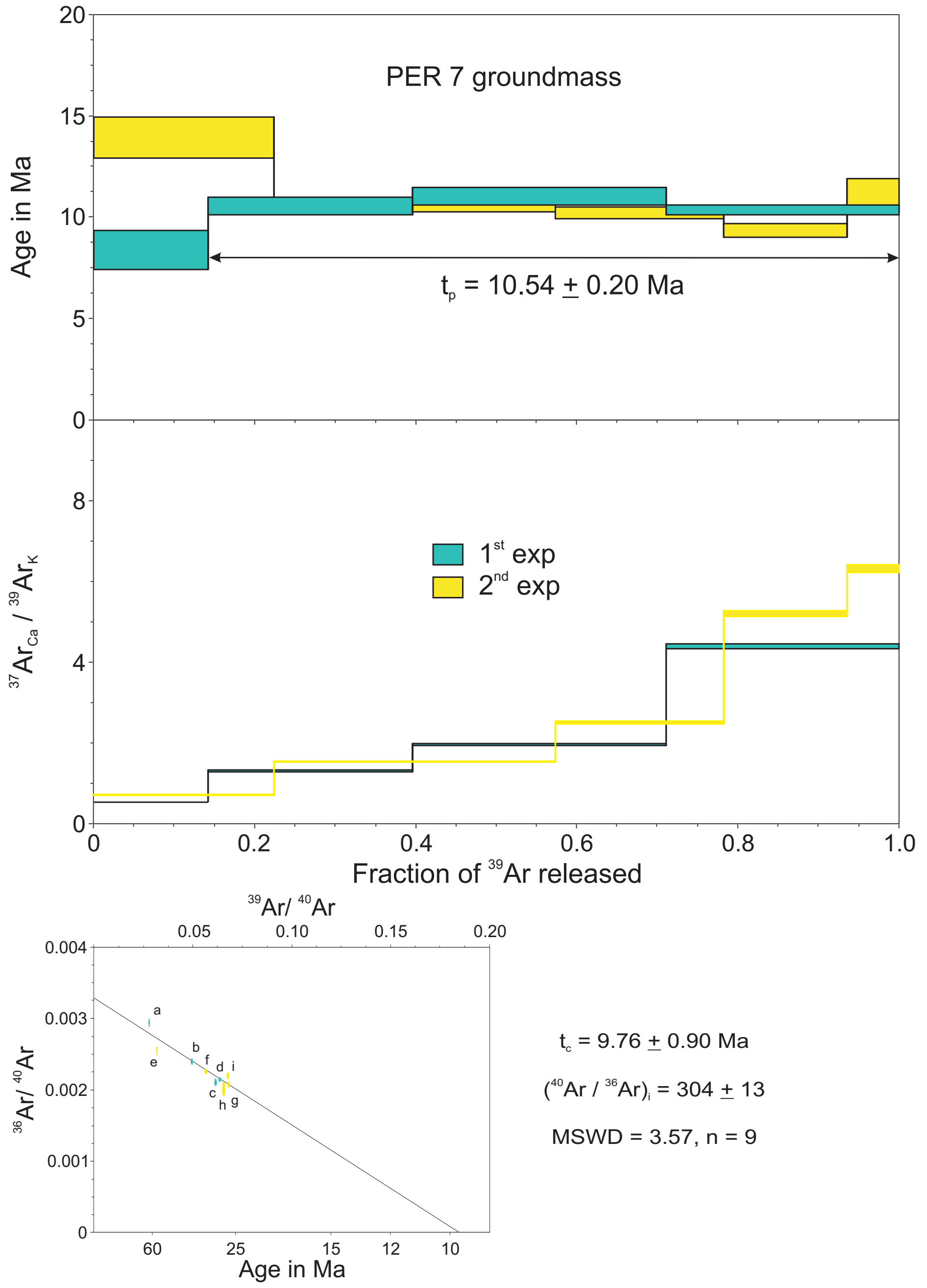

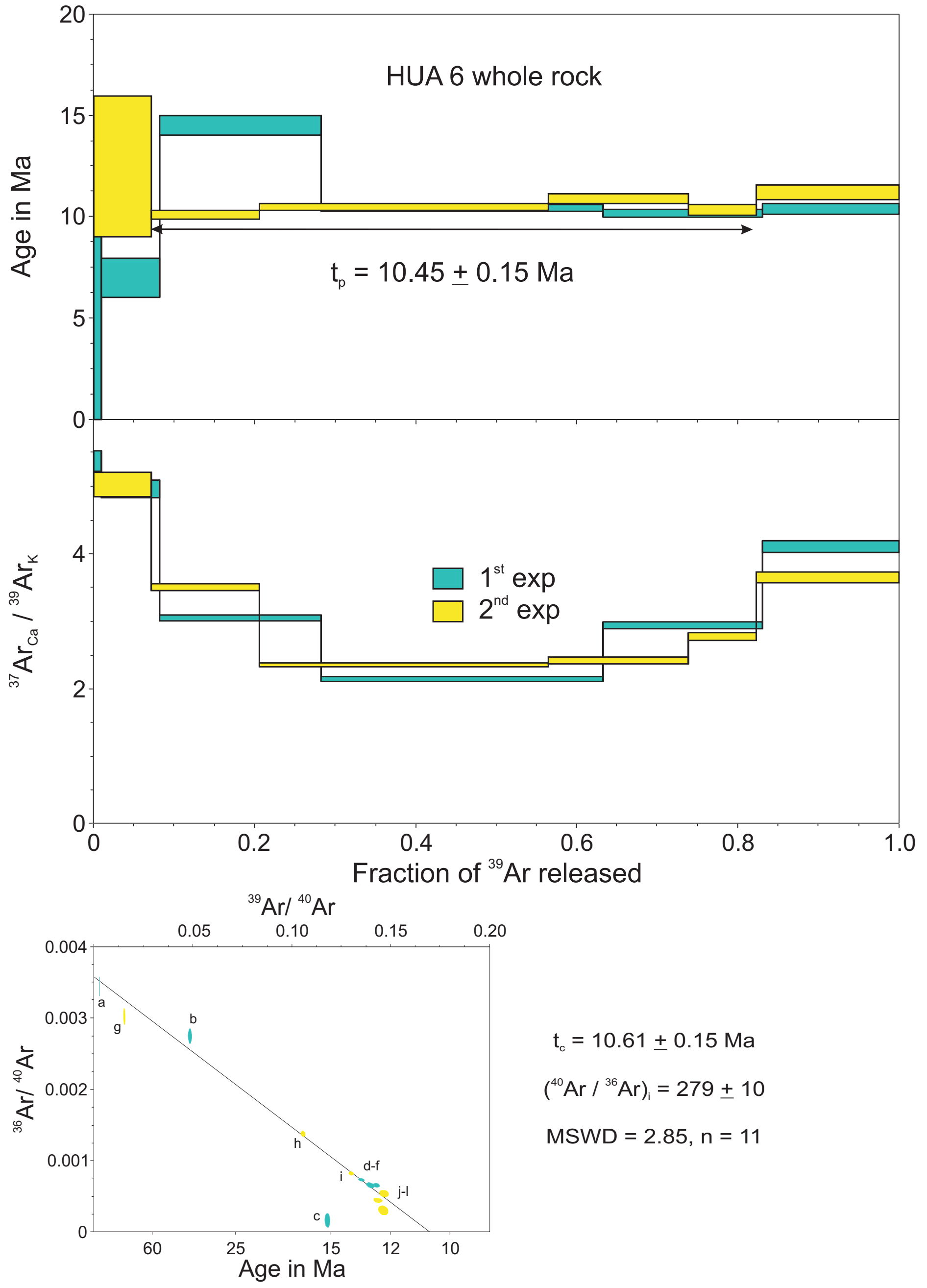

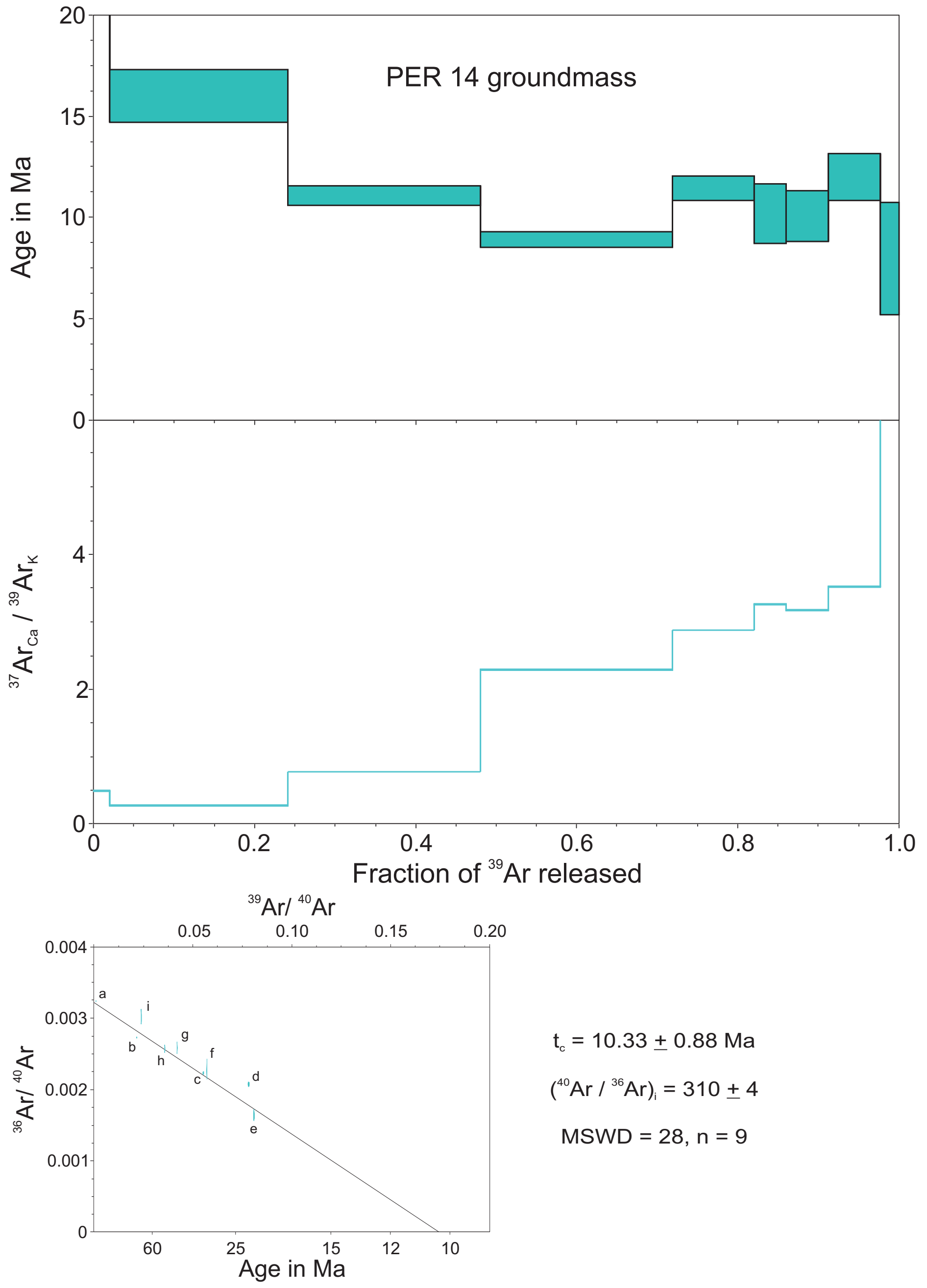

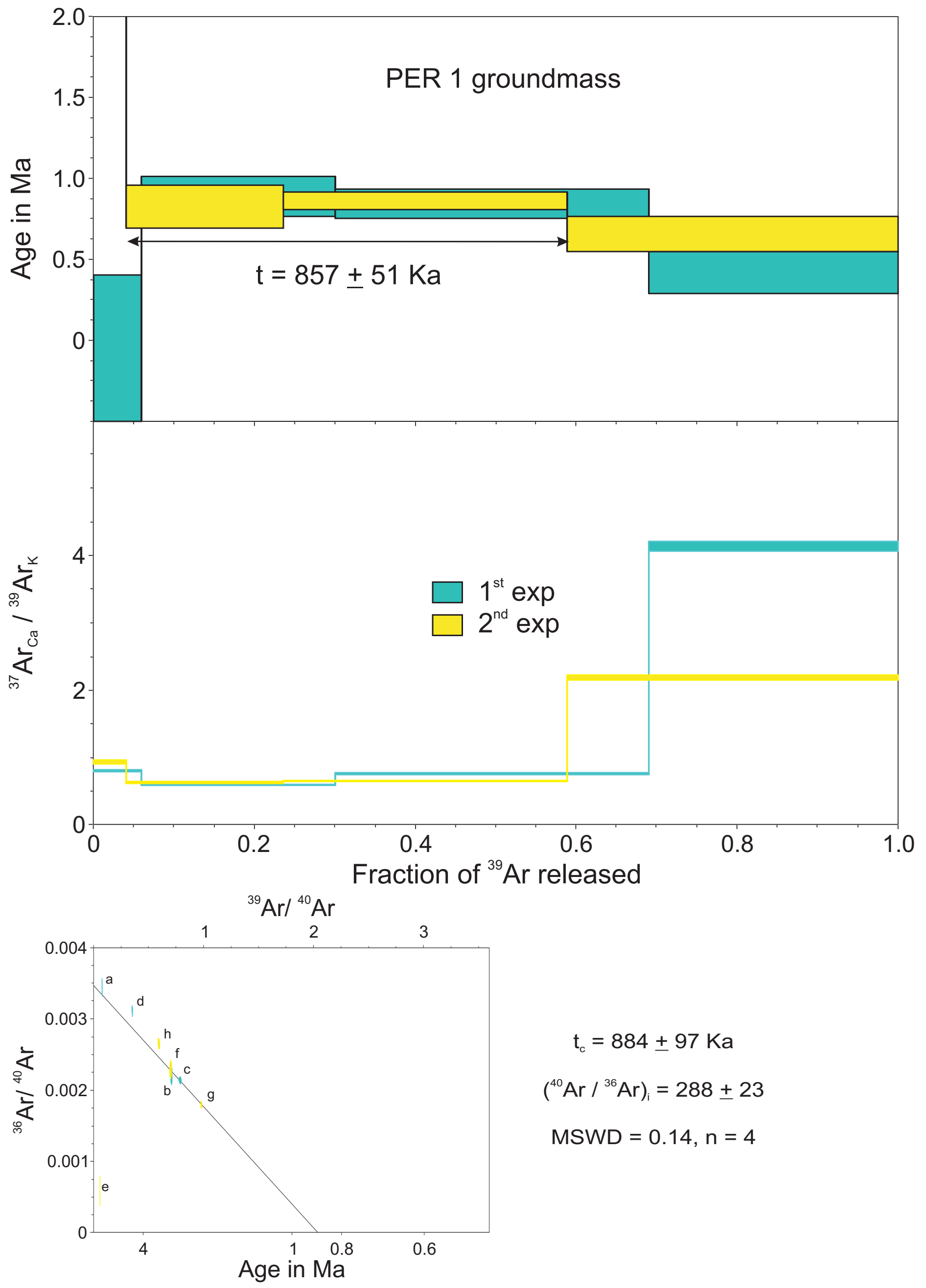

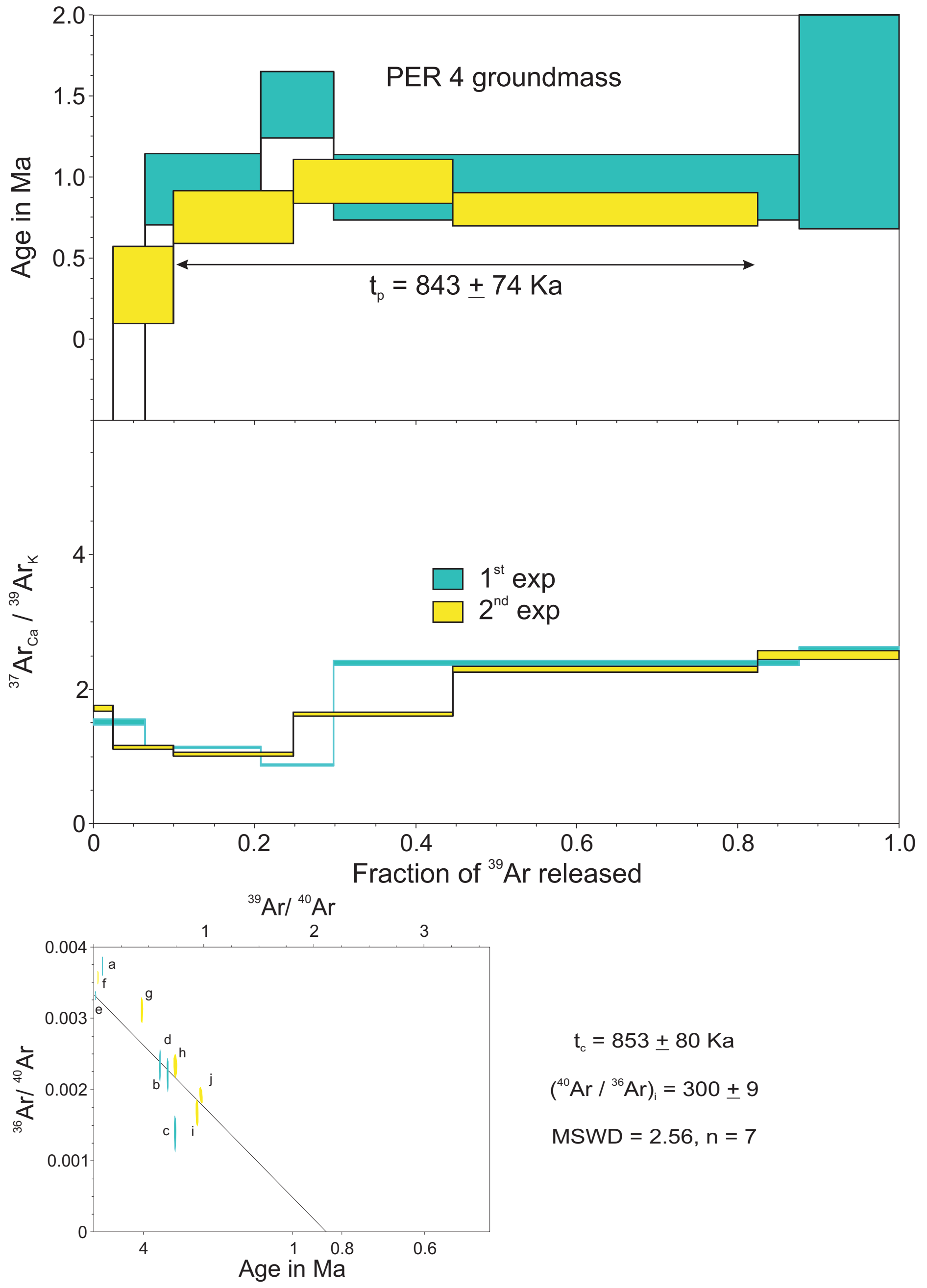

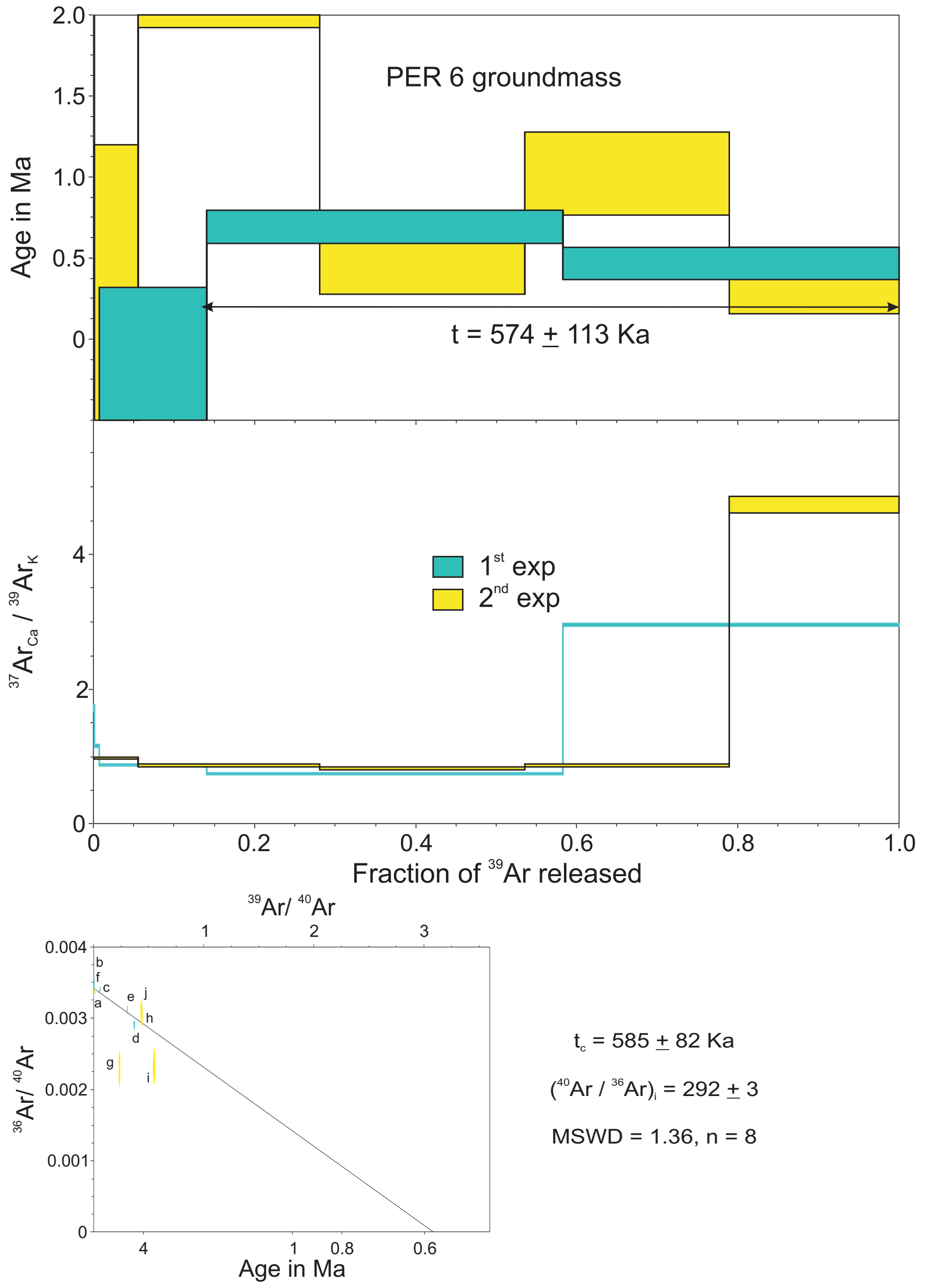


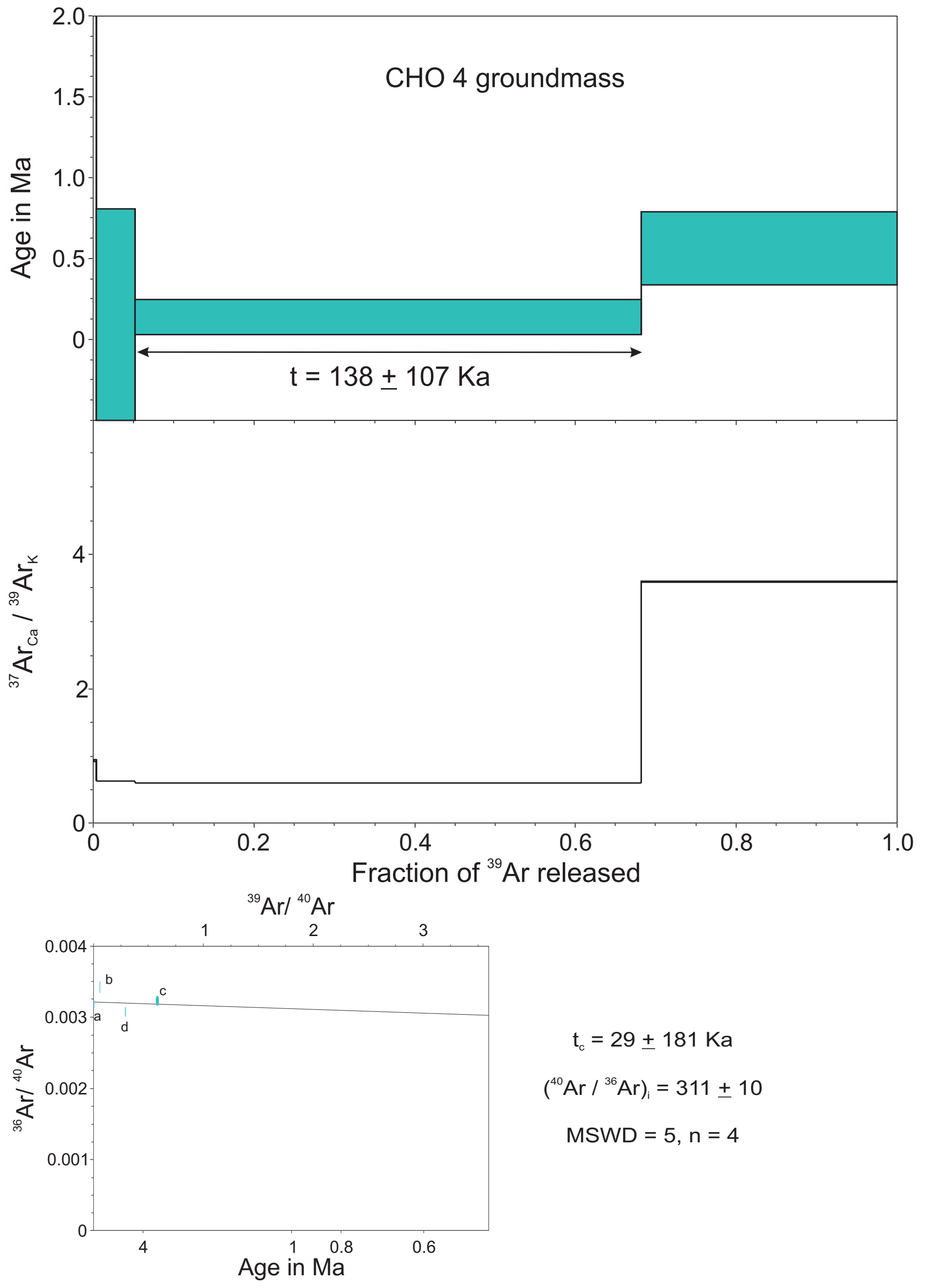

